\title{
Long-Time Asymptotics of the Nonlinear Schrödinger Equation Shock Problem
}

\author{
ROBERT BUCKINGHAM \\ University of Michigan, Ann Arbor \\ AND \\ STEPHANOS VENAKIDES \\ Duke University
}

\begin{abstract}
The long-time asymptotics of two colliding plane waves governed by the focusing nonlinear Schrödinger equation are analyzed via the inverse scattering method. We find three asymptotic regions in space-time: a region with the original wave modified by a phase perturbation, a residual region with a one-phase wave, and an intermediate transition region with a modulated two-phase wave. The leading-order terms for the three regions are computed with error estimates using the steepest-descent method for Riemann-Hilbert problems. The nondecaying initial data requires a new adaptation of this method. A new breaking mechanism involving a complex conjugate pair of branch points emerging from the real axis is observed between the residual and transition regions. Also, the effect of the collision is felt in the plane-wave state well beyond the shock front at large times. (C) 2007 Wiley Periodicals, Inc.
\end{abstract}

\section{Contents}

1. Introduction

2. Solution in the Plane-Wave Region

3. Solution in the Residual Region

4. Solution in the Transition Region

1386

Appendix A. The Forward Scattering Problem 1402

Appendix B. The Plane-Wave Region Parametrix 1406

Bibliography

\section{Introduction}

We consider the one-dimensional, cubic, focusing, nonlinear Schrödinger equation (NLS)

$$
i q_{t}+\frac{1}{2} q_{x x}+|q|^{2} q=0
$$

Communications on Pure and Applied Mathematics, Vol. LX, 1349-1414 (2007)

(C) 2007 Wiley Periodicals, Inc. 


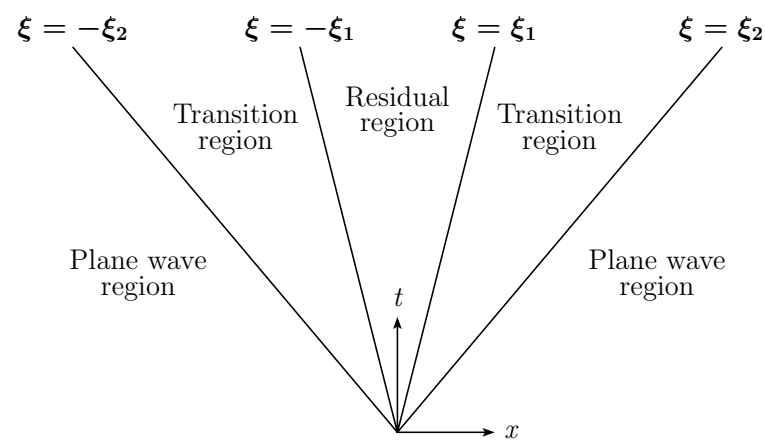

FIGURE 1.1. The three solution regions for the NLS shock problem.

with nondecaying initial data

$$
q(x, 0)=A e^{-i \mu|x|} .
$$

Here $A$ and $\mu$ are positive real constants. The initial data is interpreted as two plane waves colliding at the origin at time $t=0$.

This choice of initial data was inspired by the Toda shock problem studied by Venakides, Deift, and Oba [16]. The Toda lattice is an integrable system modeling an infinite chain of particles with nearest-neighbor interactions. Evenly spaced particles were taken to impinge on the origin at a constant speed at time $t=0$. Letting $n$ represent particle number, it was found that for large $|n / t|$, the solution behaved as in the initial state. Then at a certain value of $|n / t|$ the system entered a new solution region where the effect of the shock was being felt. Finally, for $|n / t|$ small enough, the system settled into a quiescent state when the initial speed was below a critical value or displayed nearest-neighbor oscillations when the speed was supercritical. The modulation equations for the NLS are elliptic, as opposed to hyperbolic for the Toda lattice. Still, the overall structure of the solution is similar for both problems, with three distinct solution regions.

\subsection{Main Results}

The leading-order term of the long-time limit of $q(x, t)$ behaves differently in three different regions depending on the magnitude of $\xi=x / t$ : the plane-wave region $\left(|\xi| \geq \xi_{2}\right)$, the transition region $\left(\xi_{1}<|\xi|<\xi_{2}\right)$, and the residual region in which the solution has settled to its final form $\left(|\xi| \leq \xi_{1}\right)$. See Figure 1.1. The variables $\xi_{1}$ and $\xi_{2}$ are defined in terms of $A$ and $\mu$ by the system (3.29) and by equation (2.3). Only nonnegative $x$ are considered since the problem is symmetric. The solution is given in the three regions by Theorems 1.1, 1.2, and 1.3.

THEOREM 1.1 In the plane-wave region $\left(|\xi| \geq \xi_{2}\right.$, with $\xi_{2}$ defined by equation (2.3)), for $x \geq 0$ and $t \rightarrow \infty$,

$$
q(x, t)=A e^{-i\left[\left(\mu|\xi|+\mu^{2} / 2-A^{2}\right) t-2 g(\infty)\right]}+O\left(t^{-1 / 2}\right),
$$


where $g(\infty)$ is given by equation (2.25) and depends only on $\xi$ and the parameters $A$ and $\mu$.

THEOREM 1.2 In the residual region $\left(|\xi| \leq \xi_{1}\right.$, with $\xi_{1}$ defined by the system (3.29)), for $x \geq 0$ and $t \rightarrow \infty$,

$$
q(x, t)=2 A \frac{\theta\left(\frac{\Omega_{L}}{2 \pi} t+\frac{\omega_{L}}{2 \pi}-u(\infty)+d\right) \theta(u(\infty)+d)}{\theta\left(\frac{\Omega_{L}}{2 \pi} t+\frac{\omega_{L}}{2 \pi}+u(\infty)+d\right) \theta(-u(\infty)+d)} e^{i H(t)}+O\left(t^{-1 / 2}\right),
$$

where $H(t)$ depends affinely on $t$. Specifically,

$$
H(t)=\left[2 G(\infty)-\mu|\xi|-\frac{\mu^{2}}{2}+A^{2}\right] t+2 g(\infty)
$$

Here $\theta$ is a standard one-phase theta-function defined by equation (3.43). $\Omega_{L}$ and $\omega_{L} \in \mathbb{R}$ are given by equations (3.25) and (3.36), respectively. $u(\infty), d$, $G(\infty)$, and $g(\infty) \in \mathbb{C}$ are given by equations (3.47), (3.58), (3.26), and (3.35), respectively. All of these quantities depend only on $\xi$ and the parameters $A$ and $\mu$.

THEOREM 1.3 In the transition region $\left(\xi_{1}<|\xi|<\xi_{2}\right.$, with $\xi_{1}$ and $\xi_{2}$ defined by the system (3.29) and equation (2.3)), for $x \geq 0$ and $t \rightarrow \infty$,

$q(x, t)=(2 A+\Im(\alpha)) \frac{\theta\left(\frac{\boldsymbol{\Omega}}{2 \pi} t+\frac{\omega}{2 \pi}-\boldsymbol{u}(\infty)+\boldsymbol{d}\right) \theta(\boldsymbol{u}(\infty)+\boldsymbol{d})}{\theta\left(\frac{\boldsymbol{\Omega}}{2 \pi} t+\frac{\omega}{2 \pi}+\boldsymbol{u}(\infty)+\boldsymbol{d}\right) \theta(-\boldsymbol{u}(\infty)+\boldsymbol{d})} e^{i H(t)}+O\left(t^{-1 / 2}\right)$, where $H(t)$ depends affinely on $t$. Specifically,

$$
H(t)=\left[2 G(\infty)-\mu|\xi|-\frac{\mu^{2}}{2}+A^{2}\right] t+2 g(\infty) .
$$

Here $\theta$ is a standard two-phase theta function defined by equation (4.28). $\boldsymbol{\Omega}$ and $\omega \in \mathbb{R}^{2}$ are defined by equations (4.33) and (4.34), respectively. $\alpha, G(\infty)$, and $g(\infty) \in \mathbb{C}$ are given by equations (4.12)-(4.15), (4.16), and (4.20), respectively. $\boldsymbol{u}(\infty)$ and $\boldsymbol{d} \in \mathbb{C}^{2}$ are given by equations (4.32) and (4.40), respectively. All of these quantities depend only on $\xi$ and the parameters $A$ and $\mu$.

\subsection{Discussion of Results}

In the long-time limit, the leading-order solution to the NLS (1.1) with shock initial condition (1.2) exhibits three qualitatively distinct behaviors depending on $\xi=x / t$. For large enough $\xi$, the leading-order solution is a plane wave perturbed by a phase shift that decays as $|\xi| \rightarrow \infty$. Note that if the initial condition to (1.1) was given by $q(x, 0)=A e^{-i \mu x}$, then the solution would be $q(x, t)=$ $A e^{-i\left(\mu \xi+\mu^{2} / 2-A^{2}\right) t}$. For $x>0$, this is the same as the leading-order result in Theorem 1.1 without the phase shift $g(\infty)$. This shift shows that, at large times, the solution feels the effect of the shock even beyond the outer caustic lines $\xi= \pm \xi_{2}$, a result of the nonhyperbolicity of the problem.

Inside the outer caustic lines the solution enters a transition region with two nonlinear phases. From the Riemann-Hilbert point of view, this change occurs 
when a contour of zero imaginary phase collides with a branch cut off the real axis. Inside the inner caustic lines at $\xi= \pm \xi_{1}$ the solution enters a residual state with one nonlinear phase. This change occurs when a contour of zero imaginary phase collides with the imaginary axis. This breaking mechanism is previously unseen for the NLS. A genus change when contours of zero imaginary phase collide off the real axis was seen in [15].

Our main technique is the Deift-Zhou nonlinear steepest-descent method for Riemann-Hilbert problems (see Section 1.4). The initial condition (1.2) does not decay for large $|x|$ as $|q(x, 0)|=A$ for all $x$. This introduces jumps off the real axis and necessitates a new adaptation of the nonlinear steepest-descent method.

\subsection{The Inverse Scattering Method}

Our solution uses the inverse scattering method for integrable systems such as the NLS. Integrable systems have the remarkable attribute that they are the compatability condition for a system of linear differential or difference equations known as the Lax pair. The latter was introduced by Lax [13] following the integration of the Korteweg-de Vries equation by Gardner, Greene, Kruskal, and Miura [12]. The Lax pair we use for the NLS is

$$
\begin{aligned}
W_{x} & =-i \widehat{B} W, \\
W_{t} & =-i \widehat{A} W,
\end{aligned}
$$

with

$$
\widehat{B}=\left[\begin{array}{rr}
z & q \\
\bar{q} & -z
\end{array}\right], \quad \widehat{A}=\left[\begin{array}{cc}
z^{2}-\frac{1}{2}|q|^{2} & z q+\frac{1}{2} i q_{x} \\
z \bar{q}-\frac{1}{2} i \bar{q}_{x} & -z^{2}+\frac{1}{2}|q|^{2}
\end{array}\right],
$$

and was discovered by Zakharov and Shabat [17]. Their method was generalized by Ablowitz, Kaup, Newell, and Segur [1].

The inverse scattering method in our problem reconstructs the solution $q(x, t)$ from the associated scattering data $r(z, t)$. The scattering data $r(z, 0)$ at time $t=0$ is found using equation (1.3). The key is that the time evolution of the scattering data can be found directly using equation (1.4) without the knowledge of $q(x, t)$. Therefore it is possible to find the scattering data $r(z, t)$ associated with the solution $q(x, t)$ at some later time $t$ without knowing $q(x, t) . r(z, t)$ is computed explicitly in Sections A.2 and A.3. The solution $q(x, t)$ is reconstructed from $r(z, t)$ by using the inverse scattering transformation. See Figure 1.2.

\subsection{Riemann-Hilbert Problems and the Nonlinear Steepest-Descent Method}

The Riemann-Hilbert approach to inverse scattering was first introduced in [14]. A multiplicative Riemann-Hilbert problem, or RHP, for an $n \times n$ matrix $M(z)$ consists of finding $M(z)$ given:

- an oriented contour $\Sigma$ such that $M(z)$ is analytic for $z \notin \Sigma$, 


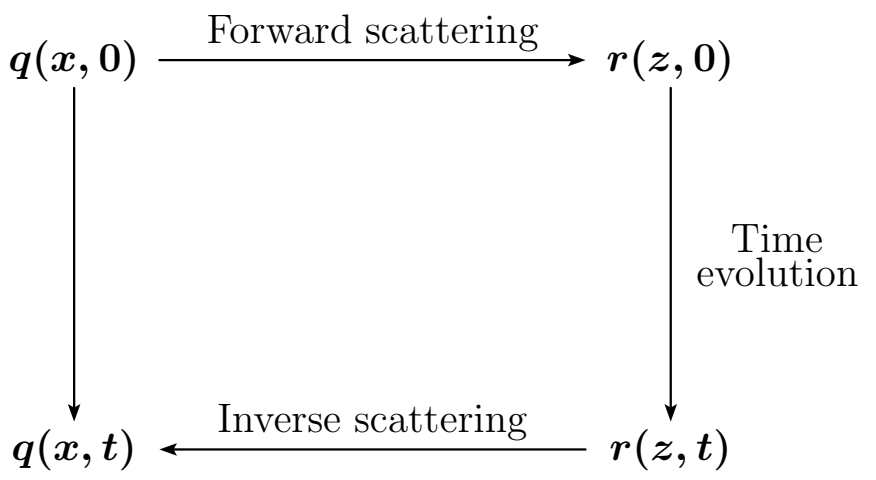

FIGURE 1.2. The inverse scattering method.

- a jump condition $M_{+}(z)=M_{-}(z) V(z)$ for $z \in \Sigma$, where $M_{+}(z)$ and $M_{-}(z)$ are the nontangential limits from the left and right of $\Sigma$, respectively, and the $n \times n$ matrix $V(z)$ is called the jump matrix, and

- a normalization, often of the form $M(z) \sim I$ as $z \rightarrow \infty$. This normalization is necessary for uniqueness because if $M$ satisfies the first two conditions, then so will $E M$ for any invertible $n \times n$ matrix $E$.

Such a problem $P$ will be represented by

$$
P:\{\Sigma, V, I \text { as } z \rightarrow \infty\} .
$$

In our case, $M^{(0)}$ is a straightforward transformation of a fundamental matrix solution of equation (1.3) satisfying appropriate conditions as $x \rightarrow \pm \infty$. As a result, the jump matrix $V^{(0)}$ can be expressed in terms of $r(z, t) . q(x, t)$ can be found from $M^{(0)}$ as shown in Section A.5. Since $r(z, t)$ is known from the forward scattering procedure, all that remains to find $q(x, t)$ is to solve the RHP for $M^{(0)}$. Solving the RHP will constitute the bulk of this work.

To analyze the RHP $P^{(0)}$ for $M^{(0)}$ in the long-time limit, we use the nonlinear steepest-descent method introduced by Deift and Zhou $[8,10]$ and extended by Deift, Venakides, and Zhou [6, 7] to treat problems in which the asymptotic waveform is fully nonlinear. This method finds asymptotic expansions in terms of small or large parameters (in this case, large $t$ ). The main technique is to factor jump matrices and deform contours such that the jump decays to the identity for large time. Then the matrix satisfying the new RHP is approximately analytic across this contour, which may be disregarded in finding the highest-order solution.

For example, consider $M^{(0)}(z)$ with jump $V^{(0)}$ across $\Sigma^{(0)}$. Assume $V^{(0)}$ has a decomposition

$$
V^{(0)}=V_{1} V_{2} V_{3}
$$




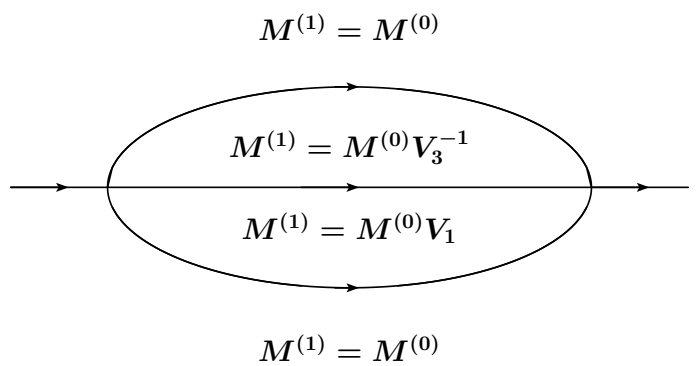

FIGURE 1.3. Sample deformation of the contour $\Sigma^{(0)}$.

into invertible factors. Along a segment split $\Sigma^{(0)}$ into three and deform one contour to the left and one to the right according to the orientation of $\Sigma$. Define a new matrix $M^{(1)}(z)$ by

$$
M^{(1)}= \begin{cases}M^{(0)} V_{3}^{-1} & \text { inside new contour to the left, } \\ M^{(0)} V_{1} & \text { inside new contour to the right, } \\ M^{(0)} & \text { elsewhere, }\end{cases}
$$

as shown in Figure 1.3. Across the left contour,

$$
\begin{aligned}
M_{+}^{(1)} & =M_{+}^{(0)} \\
& =M_{+}^{(0)} V_{3}^{-1} V_{3} \\
& =M_{-}^{(1)} V_{3} .
\end{aligned}
$$

Therefore $M^{(1)}$ has a jump of $V_{3}$ across the left contour. Similarly, $M^{(1)}$ has a jump of $V_{1}$ across the right contour and a jump of $V_{2}$ across the center contour. Therefore, if $M^{(1)}$ can be found that satisfies the RHP with the deformed contour, the solution $M^{(0)}$ to the original problem can be found from equation (1.7). We say two RHPs are equivalent if, given the solution to one problem, the solution to the other can be found by a change of variables.

In this work we start with the RHP $P^{(0)}$ defined in Section 1.5 and perform a series of transformations $P^{(0)} \rightarrow P^{(1)} \rightarrow P^{(2)} \rightarrow \cdots$ to obtain equivalent RHPs. We peel off successive layers of the RHP, eventually arriving at an exactly solvable model problem that gives the leading-order term of $q(x, t)$.

\subsection{The RHP for the NLS Shock Problem}

The fundamental solution $M^{(0)}$ to the Lax pair for the NLS shock problem is defined in Section A.4. Define

$$
\lambda_{L}(z)=\left(\left(z+\frac{\mu}{2}\right)^{2}+A^{2}\right)^{1 / 2}, \quad \lambda_{R}(z)=\left(\left(z-\frac{\mu}{2}\right)^{2}+A^{2}\right)^{1 / 2} .
$$




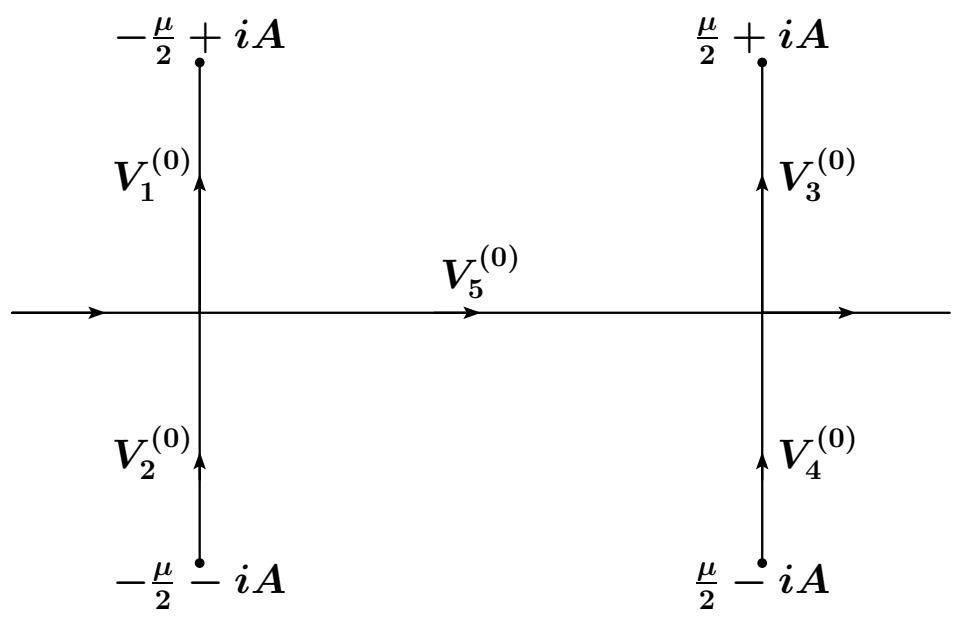

FIGURE 1.4. The RHP $P^{(0)}$.

The $\lambda_{L}$ branch cut is taken to be the vertical line segment from $-\mu / 2-i A$ to $-\mu / 2+i A$, and the $\lambda_{R}$ branch cut is the vertical line segment from $\mu / 2-i A$ to $\mu / 2+i A$. The sheets are chosen so that $\lambda_{L}, \lambda_{R} \rightarrow z$ as $z \rightarrow \infty$. Furthermore, $\lambda_{L}(z)=\lambda_{L-}(z)$ and $\lambda_{R}(z)=\lambda_{R_{-}}(z)$ on their respective branch cuts. The subscripts $L$ and $R$ refer to the left and right branch cuts, respectively. Also define

$$
\begin{gathered}
\xi=\frac{x}{t}, \\
\Lambda(z)=\frac{\lambda_{R}+(z-\mu / 2)}{A}, \\
D(z)=\frac{2 \lambda_{R}^{2}-2(z-\mu / 2) \lambda_{R}}{A^{2}} \\
f(z, \xi)=2 \lambda_{R} \xi+2\left(z+\frac{\mu}{2}\right) \lambda_{R}, \\
\rho(z)=\frac{-\lambda_{R}-(z-\mu / 2)}{A} \frac{\left(\lambda_{R}-(z-\mu / 2)-\lambda_{L}+(z+\mu / 2)\right)}{\left(\lambda_{R}+(z-\mu / 2)+\lambda_{L}-(z+\mu / 2)\right)} .
\end{gathered}
$$

Now $M^{(0)}(z ; x, t)$ is the solution to the RHP

$$
P^{(0)}:\left\{\Sigma^{(0)}=\mathbb{R} \cup\left(\lambda_{L} \text { cut }\right) \cup\left(\lambda_{R} \text { cut }\right), V^{(0)}, I \text { as } z \rightarrow \infty\right\},
$$


with $V^{(0)}$ given by Figure 1.4 and

$$
\begin{gathered}
V_{1}^{(0)}(z)=\left[\begin{array}{cc}
1 & 0 \\
\frac{1}{D}\left(\rho_{+}-\rho_{-}\right) e^{i f t} & 1
\end{array}\right], \quad V_{2}^{(0)}(z)=\left[\begin{array}{cc}
1 & \frac{1}{D}\left(-\rho_{+}+\rho_{-}\right) e^{-i f t} \\
0 & 1
\end{array}\right], \\
V_{3}^{(0)}(z)=\left[\begin{array}{cc}
-\Lambda_{+} \rho_{+} e^{i f_{+} t} & D_{-} \Lambda_{-} \\
0 & -\Lambda_{-} \rho_{-} e^{i f_{-} t}
\end{array}\right], \\
V_{4}^{(0)}(z)=\left[\begin{array}{cc}
-\Lambda_{-} \rho_{-} e^{-i f_{-} t} & 0 \\
-D_{-} \Lambda_{-} & -\Lambda_{+} \rho_{+} e^{-i f_{+} t}
\end{array}\right], \quad V_{5}^{(0)}(z)=\left[\begin{array}{cc}
\frac{1+\rho^{2}}{D^{i f t}} & \rho e^{-i f t} \\
\rho e^{-1} & D
\end{array}\right] .
\end{gathered}
$$

These jump matrices satisfy the symmetry relation $(\overline{V(\bar{z})})^{\top} V(z)=I$.

\subsection{The Controlling Phase Functions $f$ and $h$ and Overview of the Procedure}

The sign structure of $\Im(f)$ (which depends on $\xi$ ) controls where in the complex plane the factored jump matrices will decay or blow up. Assume $x>0$. For the plane-wave region $\left(\xi>\xi_{2}\right)$, the jump matrices will, after appropriate factorizations and deformations, decay everywhere except on a single band along the $\lambda_{R}$ cut. See Figure 1.5 for an overview of the intermediate tranformations in the plane-wave region. The model problem that gives the leading-order behavior of $q(x, t)$ consists of only the $\lambda_{R}$ cut. In the plane-wave region the real point $z_{0}$ is the stationary point of $f(z)$.

For $\xi<\xi_{2}$, this procedure breaks down, and blowup can no longer be avoided simply by contour deformations. We use the $g$-function mechanism to remove the exponential growth. The controlling phase function will now be a function $h(z)$, and $z_{0}$ will be the stationary phase point of $h(z)$. Different transformations will be used in the transition and residual regions. Figure 1.6 shows the evolution of the final transformed problem as $\xi$ varies. At $\xi=\xi_{2}$, two contributing bands are born that lie along the $\lambda_{L}$ branch cut and whose jumps cancel each other. As $\xi$ decreases, one band stays on the $\lambda_{L}$ cut, and the other moves to the right and decreases in length. There are three bands in the model problem in the transition region $\left(\xi_{1}<\xi<\xi_{2}\right)$.

At $\xi=\xi_{1}$, the center band disappears into the real axis, and the problem enters the residual region $\left(\xi<\xi_{1}\right)$ with two bands in the model problem. The genus of the Riemann surface associated with the solution changes from 2 to 1 when the contour of zero imaginary phase collides with the real axis (see Figure 3.3).

Note that we start with the plane-wave region in Section 2, but then proceed to the residual region in Section 3 and then to the transition region in Section 4 because the problem with two bands is less complicated than the problem with three bands. 


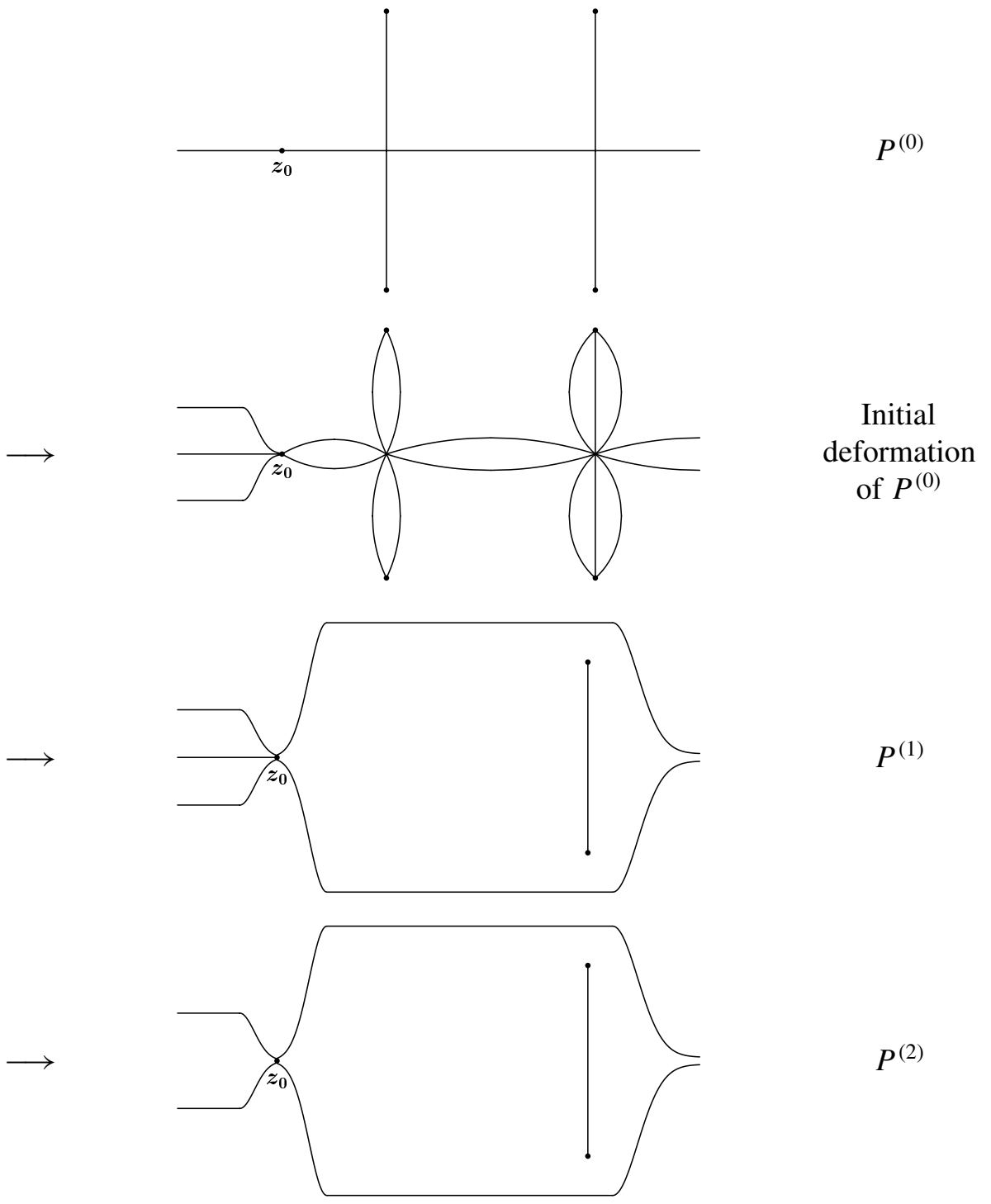

FIGURE 1.5. The series of intermediate transformations of $P^{(0)}$ in the plane-wave region.

The different deformations shown in Figure 1.6 use different factorizations of the jump matrices $V^{(0)}$. The factorizations we use are

$$
\begin{aligned}
{\left[\begin{array}{cc}
1+a b & a \\
b & 1
\end{array}\right] } & =\left[\begin{array}{cc}
1 & 0 \\
\frac{b}{1+a b} & 1
\end{array}\right]\left[\begin{array}{cc}
1+a b & 0 \\
0 & \frac{1}{1+a b}
\end{array}\right]\left[\begin{array}{cc}
1 & \frac{a}{1+a b} \\
0 & 1
\end{array}\right] \\
& =\left[\begin{array}{ll}
1 & a \\
0 & 1
\end{array}\right]\left[\begin{array}{ll}
1 & 0 \\
b & 1
\end{array}\right] \quad(a b \neq-1),
\end{aligned}
$$




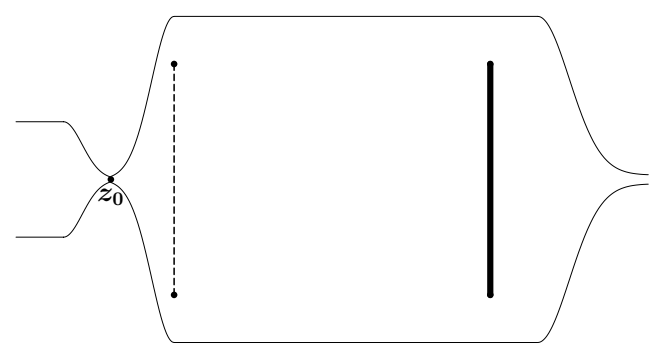

\section{Plane-wave region}

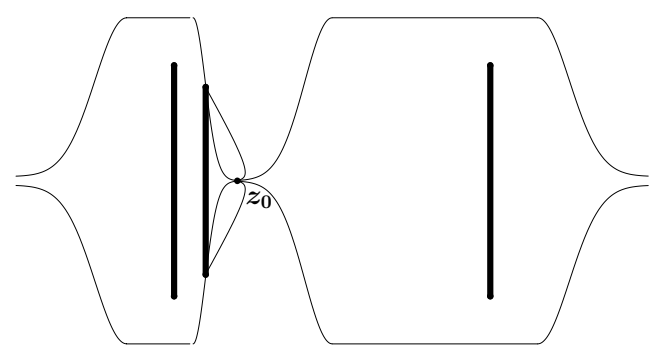

Transition region

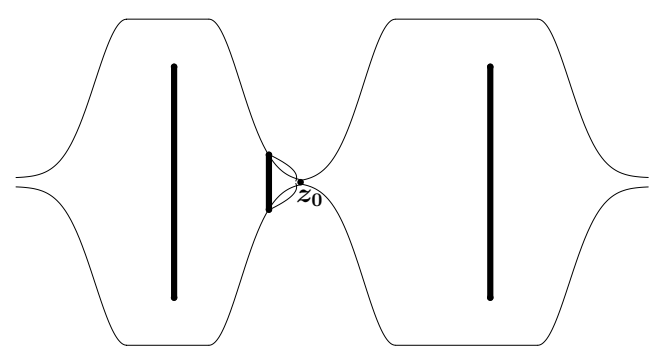

Transition region

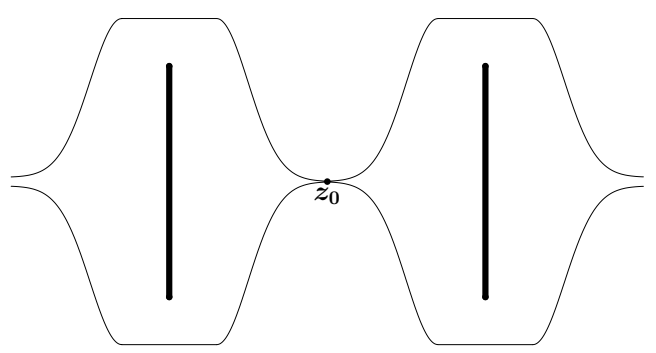

Residual region

FIGURE 1.6. The final transformed RHP for decreasing values of $\xi$. The jump on the thin contours decay in time; the jump on the thick contours contribute to the leading-order solution. The dashed line in the first picture is not part of the RHP but indicates the $\lambda_{L}$ cut.

$$
\begin{aligned}
& {\left[\begin{array}{cc}
1 & 0 \\
a+b & 1
\end{array}\right]=\left[\begin{array}{ll}
1 & 0 \\
a & 1
\end{array}\right]\left[\begin{array}{ll}
1 & 0 \\
b & 1
\end{array}\right],} \\
& {\left[\begin{array}{cc}
a & 0 \\
-b & a^{-1}
\end{array}\right]=\left[\begin{array}{cc}
1 & -a b^{-1} \\
0 & 1
\end{array}\right]\left[\begin{array}{cc}
0 & b^{-1} \\
-b & 0
\end{array}\right]\left[\begin{array}{cc}
1 & -a^{-1} b^{-1} \\
0 & 1
\end{array}\right] \quad(a, b \neq 0),}
\end{aligned}
$$

and their transposes. 


\section{Solution in the Plane-Wave Region}

The solution to the shock problem is simplest when the jumps $V_{1}^{(0)}$ and $V_{2}^{(0)}$ on the $\lambda_{L}$ branch cut decay to the identity. Then the problem reduces (modulo higherorder terms) to a single band on the $\lambda_{R}$ branch cut. In this case the leading-order solution is a plane wave with a perturbation in the phase, and the problem is said to be in the plane-wave region.

\subsection{Overview of the Solution in the Plane-Wave Region}

We begin by studying $\Im(f)$ in Section 2.2 to find the $\xi$ that comprise the planewave region. We then do a series of transformations of the RHP $P^{(0)}$ to find the leading-order contribution.

- $P^{(0)} \rightarrow P^{(1)}: P^{(1)}$ is found in Section 2.3 by factoring $V^{(0)}$ and deforming $\Sigma^{(0)} \cdot V^{(1)}$ decays uniformly in $t$ to the identity off $\left(-\infty, z_{0}\right) \cup\left(\lambda_{R}\right.$ branch cut) and outside of a small neighborhood of $z_{0}$.

- $P^{(1)} \rightarrow P^{(2)}: P^{(2)}$ is defined in Section 2.4 by removing the jump across $\left(-\infty, z_{0}\right)$ using $\delta(z)$.

- $P^{(2)} \rightarrow P^{(3)}$ : The factor of $D(z)$ in the jump matrices is removed by the definition of $P^{(3)}$ in Section 2.5 .

- $P^{(3)} \rightarrow P^{(4)}$ : The definition of $P^{(2)}$ introduces terms involving $\delta(z)$ to the jump on the $\lambda_{R}$ branch cut. $P^{(4)}$ removes these terms by the use of a $g$-function in Section 2.6.

The leading-order term of $q(x, t)$ can now be found by solving the model RHP $P^{(\text {mod) }}$, which disregards all of the contours of $\Sigma^{(3)}$ except the $\lambda_{R}$ branch cut. $P^{\text {(mod) }}$ is defined and solved in Section 2.7. However, the nonuniform decay near $z_{0}$ must be taken into account for the error estimate. Therefore in Section $2.8, P^{(3)}$ is split into an approximate RHP $P^{(\mathrm{app})}$ with jumps on the $\lambda_{R}$ branch cut and near $z_{0}$, and an error RHP $P^{(\text {err })}$ with uniformly decaying jumps. The error estimate is proved in Section 2.9.

\subsection{Behavior of $\Im(f)$}

From equations (1.15), $V_{1}^{(0)}$ decays to the identity when $\Im(f)>0$ everywhere along the $\lambda_{L}$ branch cut in the upper half-plane. Write $z=\eta+i v$ where $\eta, v \in \mathbb{R}$. For $\eta<\frac{\mu}{2}$ and $0<v<<1$,

$$
\Im(f) \sim-2 v \frac{\left(\eta-\frac{\mu}{2}\right)\left(\xi+\eta+\frac{\mu}{2}\right)}{\sqrt{A^{2}+(\eta-\mu / 2)^{2}}}-2 v \sqrt{A^{2}+\left(\eta-\frac{\mu}{2}\right)^{2}} .
$$

Therefore, immediately above the real axis, $\operatorname{sgn}(\Im(f))=0$ when

$$
\eta=\eta_{ \pm}=\frac{\mu-\xi \pm \sqrt{(\xi+\mu)^{2}-8 A^{2}}}{4} .
$$




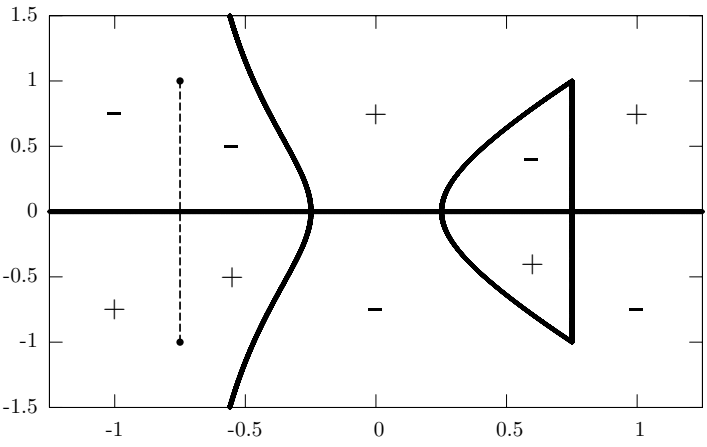

(a) $\xi=1.5$ (outside plane-wave region)

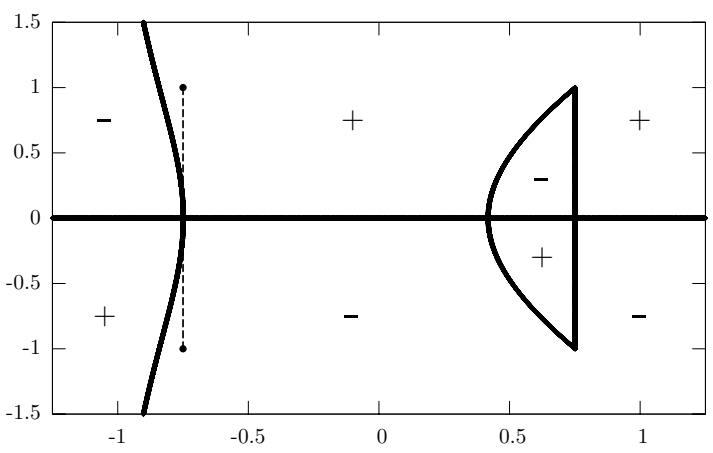

(b) $\xi=\xi_{2} \approx 2.17$ (borderline between transition and plane-wave regions)

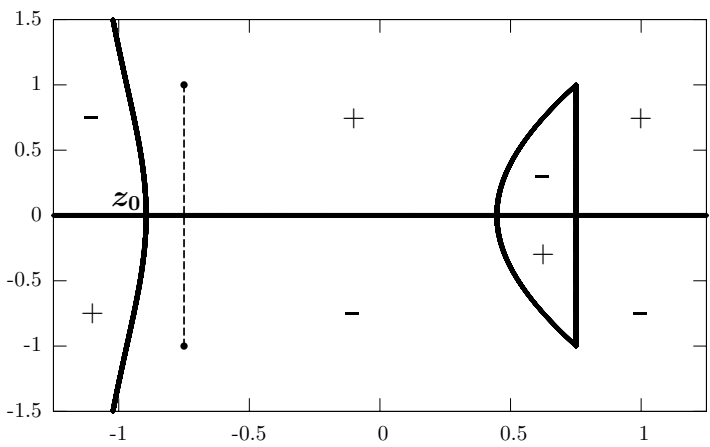

(c) $\xi=2.4$ (plane-wave region)

FIGURE 2.1. Sign structure of $\Im(f)$ for $A=1, \mu=1.5$. The dashed line does not participate in the structure but demarcates the $\lambda_{L}$ cut.

As seen in Figure 2.1, $\Im(f)>0$ everywhere along the $\lambda_{L}$ branch cut in the upper half-plane when $\eta_{-}<-\frac{\mu}{2}$. Therefore the problem is in the plane-wave region 


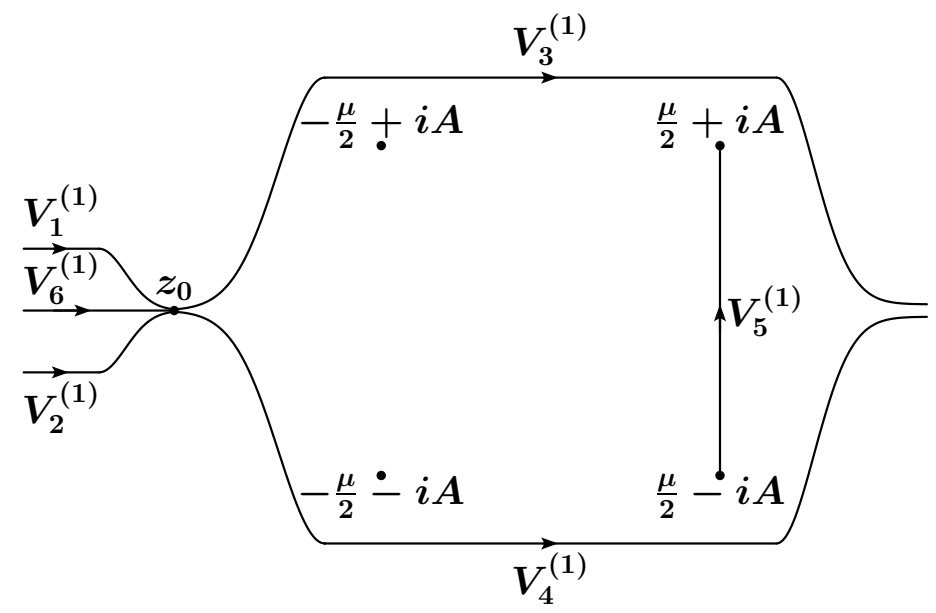

FIGURE 2.2. The RHP $P^{(1)}$ for the plane-wave region.

when

$$
\xi \geq \xi_{2}=\frac{\mu^{2}+A^{2}}{\mu}
$$

The Schwarz symmetry of $f$ guarantees that $V_{2}^{(0)}$ will also decay to the identity for $\xi$ in the plane-wave region.

Two different factorizations of the real axis will be used in all three regions. The point at which the factorization changes will be called $z_{0}$. In the plane-wave region, define

$$
z_{0}=\frac{\mu-\xi-\sqrt{(\xi+\mu)^{2}-8 A^{2}}}{4}
$$

See Figure 2.1(c). The appropriate factorization of $V^{(0)}$ and deformation of $\Sigma^{(0)}$ will now be given for the plane-wave region.

\section{$2.3 P^{(0)} \rightarrow P^{(1)}$ : Factorization of $V^{(0)}$ and Deformation of $\Sigma^{(0)}$}

The RHP $P^{(1)}$ is given by

$$
P^{(1)}:\left\{\Sigma^{(1)} \text { (see Figure 2.2), } V^{(1)}, I \text { as } z \rightarrow \infty\right\},
$$

where 


$$
\begin{array}{rlrl}
V_{1}^{(1)} & =\left[\begin{array}{cc}
\frac{1}{D^{1 / 2}} & \frac{D^{1 / 2} \rho}{1+\rho^{2}} e^{-i f t} \\
0 & D^{1 / 2}
\end{array}\right], & V_{2}^{(1)}=\left[\begin{array}{cc}
\frac{1}{D^{1 / 2}} & 0 \\
\frac{D^{1 / 2} \rho}{1+\rho^{2}} e^{i f t} & D^{1 / 2}
\end{array}\right], \\
V_{3}^{(1)}=\left[\begin{array}{cc}
\frac{1}{D^{1 / 2}} & 0 \\
\frac{\rho}{D^{1 / 2}} e^{i f t} & D^{1 / 2}
\end{array}\right], & V_{4}^{(1)}=\left[\begin{array}{cc}
\frac{1}{D^{1 / 2}} & \frac{\rho}{D^{1 / 2}} e^{-i f t} \\
0 & D^{1 / 2}
\end{array}\right], \\
V_{5}^{(1)}=\left[\begin{array}{cc}
0 & 1 \\
-1 & 0
\end{array}\right], & V_{6}^{(1)}=\left[\begin{array}{cc}
1+\rho^{2} & 0 \\
0 & \frac{1}{1+\rho^{2}}
\end{array}\right] .
\end{array}
$$

Here the square root is chosen so $D^{1 / 2} \rightarrow 1$ as $z \rightarrow \infty$. Comparing Figures 2.1(c) and 2.2, one sees that in the plane-wave region, $\Sigma^{(1)}$ can be chosen so that $V_{i}^{(1)}$, $i=1,2,3,4$, decay as $t \rightarrow \infty$. This new RHP will be useful for analyzing the asymptotics of $q(x, t)$ for $\xi>\xi_{2}$.

CLAIM The RHPS $P^{(0)}$ and $P^{(1)}$ are equivalent.

Proof: Consider first the jump along the $\lambda_{L}$ branch cut in the upper half-plane. The matrix $V_{1}^{(0)}$ is factored as

$$
V_{1}^{(0)}=\left(V_{3-}^{(1)}\right)^{-1} V_{3+}^{(1)}
$$

Split this contour in two, keeping the new contours attached to the $\lambda_{L}$ branch cut at $-\mu / 2$ and $-\mu / 2+i A$. The right factor is the jump matrix for the contour being deformed to the left (the plus side) of the original contour, so $\rho_{+}$becomes $\rho$. Therefore the jump matrix on the left contour is $V_{3}^{(1)}$. Similarly, the left factor is the jump matrix for the contour being deformed to the right (the minus side) of the original contour, so the jump matrix on the right contour is $\left(V_{3}^{(1)}\right)^{-1}$. The other matrices are factored as follows:

$$
\begin{array}{ll}
V_{2}^{(0)}=V_{4-}^{(1)}\left(V_{4+}^{(1)}\right)^{-1} & \text { on the } \lambda_{L} \text { cut in lower half-plane, } \\
V_{3}^{(0)}=\left(V_{3-}^{(1)}\right)^{-1} V_{5}^{(1)} V_{3+}^{(1)} & \text { on the } \lambda_{R} \text { cut in upper half-plane, } \\
V_{4}^{(0)}=V_{4-}^{(1)} V_{5}^{(1)}\left(V_{4+}^{(1)}\right)^{-1} & \text { on the } \lambda_{R} \text { cut in lower half-plane, } \\
V_{5}^{(0)}=V_{2}^{(1)} V_{6}^{(1)} V_{1}^{(1)} & \text { on }\left(-\infty, z_{0}\right), \\
V_{5}^{(0)}=V_{4}^{(1)} V_{3}^{(1)} & \text { on }\left(z_{0}, \infty\right) .
\end{array}
$$

The contours are split and deformed as shown in Figure 2.3. The jump matrices $V_{1}^{(1)}, V_{2}^{(1)}, V_{3}^{(1)}$, and $V_{4}^{(1)}$ now decay in time to the identity away from the points $-\mu / 2, \mu / 2, \mu / 2+i A$, and $\mu / 2-i A$. Ideally the contour would be deformed away from these points to achieve uniform decay. However, $f$ and $\rho$ are not analytic at these points, nor at $-\mu / 2+i A$ and $-\mu / 2-i A$. Still, it is possible to lift the contour away from these points as follows.

Consider the two contours connected to $-\mu 2+i A$ and deform them so they coincide on the vertical line $\Re(z)=-\mu / 2$. Since the jump matrices on the two 


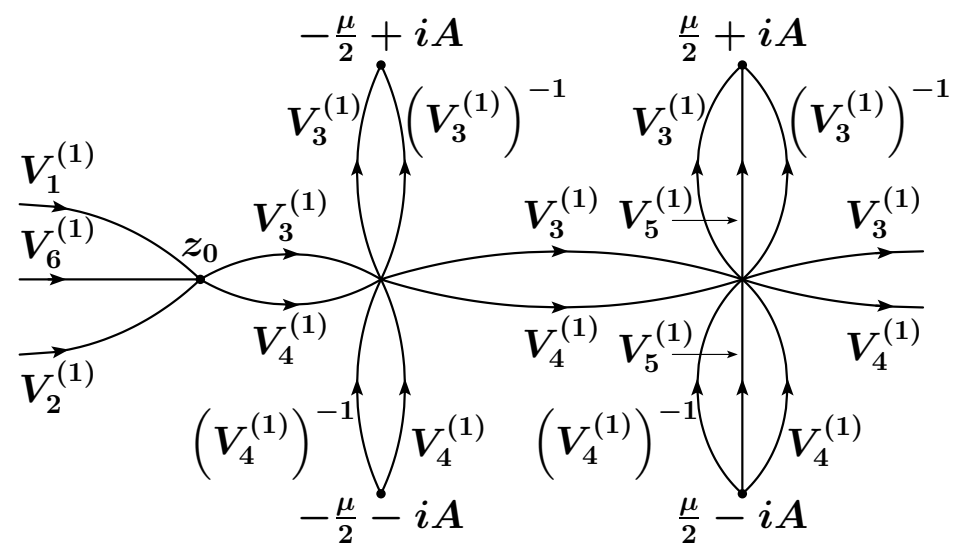

FIGURE 2.3. Initial deformation of $P^{(0)}$.
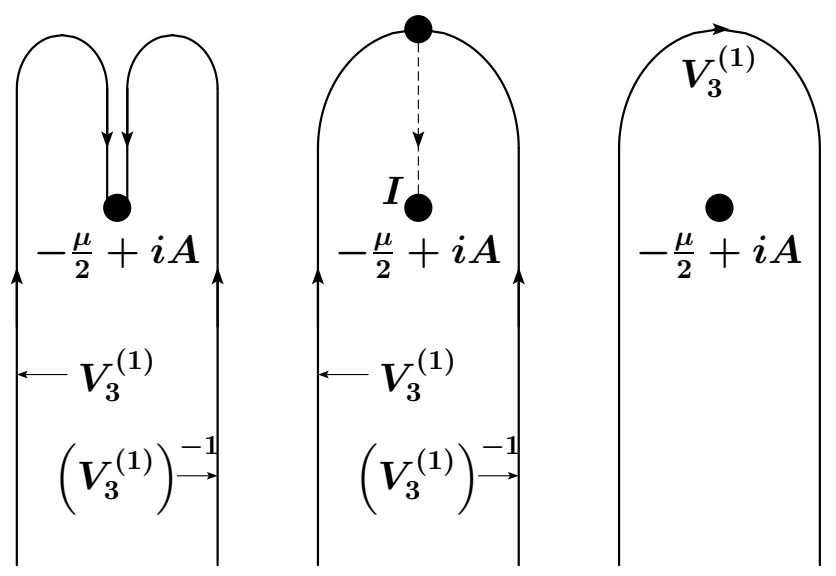

FIGURE 2.4. Three stages of the final deformation of $P^{(0)}$ near $-\mu / 2+i A$.

contours are inverses, the resulting jump matrix is the identity where the two coincide, and so this part of the contour can be removed. Finally, reorient the right contour and replace $\left(V_{3}^{(1)}\right)^{-1}$ with $V_{3}^{(1)}$. Now there is a single contour oriented left to right with jump matrix $V_{3}^{(1)}$; see Figure 2.4. A similar procedure is used on the contours at $-\mu / 2-i A,-\mu / 2, \mu / 2, \mu / 2+i A$, and $\mu / 2-i A$. Deform the loosened contours so that the contours with jumps $V_{1}^{(1)}$ and $V_{4}^{(1)}$ lie in regions where $\Im(f)<0$, and the contours with jumps $V_{2}^{(1)}$ and $V_{3}^{(1)}$ lie in regions where $\Im(f)>0$. This completes the construction of $P^{(1)}$ and establishes the claim. 


\section{$2.4 P^{(1)} \rightarrow P^{(2)}$ : Elimination of the Jump on $\left(-\infty, z_{0}\right)$}

The jump matrix $V_{6}^{(1)}$ on $\left(-\infty, z_{0}\right)$ is removed by introducing the function $\delta(z)$. Let $\delta(z)$ solve the scalar RHP

$$
P^{(\delta)}:\left\{\Sigma^{(\delta)}=\left(-\infty, z_{0}\right), V^{(\delta)}=1+\rho^{2}, I \text { as } z \rightarrow \infty\right\}
$$

Explicitly,

$$
\delta(z)=e^{\frac{1}{2 \pi i} \int_{-\infty}^{z} \frac{\ln \left(1+[\rho(\zeta)]^{2}\right)}{\zeta-z} \mathrm{~d} \zeta} .
$$

Define

$$
M^{(2)}=M^{(1)} \delta^{-\sigma_{3}}
$$

Now

$$
V^{(2)}=\delta_{-}^{\sigma_{3}} V^{(1)} \delta_{+}^{-\sigma_{3}}
$$

Specifically,

$$
\begin{array}{rlrl}
2 V_{1}^{(2)} & =\left[\begin{array}{cc}
\frac{1}{D^{1 / 2}} & \frac{\delta^{2} D^{1 / 2} \rho}{1+\rho^{2}} e^{-i f t} \\
0 & D^{1 / 2}
\end{array}\right], & V_{2}^{(2)}=\left[\begin{array}{cc}
\frac{1}{D^{1 / 2}} & 0 \\
\frac{\delta^{-2} D^{1 / 2} \rho}{1+\rho^{2}} e^{i f t} & D^{1 / 2}
\end{array}\right], \\
V_{3}^{(2)} & =\left[\begin{array}{cc}
\frac{1}{D^{1 / 2}} & 0 \\
\frac{\delta^{-2} \rho}{D^{1 / 2}} e^{i f t} & D^{1 / 2}
\end{array}\right], & V_{4}^{(2)}=\left[\begin{array}{cc}
\frac{1}{D^{1 / 2}} & \frac{\delta^{2} \rho}{D^{1 / 2}} e^{-i f t} \\
0 & D^{1 / 2}
\end{array}\right], \\
V_{5}^{(2)} & =\left[\begin{array}{cc}
0 & \delta^{2} \\
-\delta^{-2} & 0
\end{array}\right] .
\end{array}
$$

Since $\delta \sim 1$ as $z \rightarrow \infty$, the normalization of $M^{(2)}$ at infinity is unchanged from $M^{(1)} \cdot M^{(2)}$ now satisfies the RHP

$$
P^{(2)}:\left\{\Sigma^{(2)}=\Sigma^{(1)} \backslash \Sigma^{(\delta)}, V^{(2)}, I \text { as } z \rightarrow \infty\right\} .
$$

$V^{(2)}$ is defined by Figure 2.5 and equation (2.15).

\section{$2.5 P^{(2)} \rightarrow P^{(3)}$ : Removal of $D(z)$}

The factor of $D(z)$ can be removed from the jump matrices by a change of variables outside of the deformed contours. Define

$$
M^{(3)}= \begin{cases}M^{(2)} D^{\sigma_{3} / 2} & \text { in region I, } \\ M^{(2)} D^{-\sigma_{3} / 2} & \text { in region III, } \\ M^{(2)} & \text { in regions II and IV, }\end{cases}
$$

where $\sigma_{3}=\left[\begin{array}{cc}1 & 0 \\ 0 & -1\end{array}\right]$ and regions I through IV are defined in Figure 2.5. $M^{(3)}$ satisfies the RHP

$$
P^{(3)}:\left\{\Sigma^{(3)}=\Sigma^{(2)}, V^{(3)}, I \text { as } z \rightarrow \infty\right\},
$$




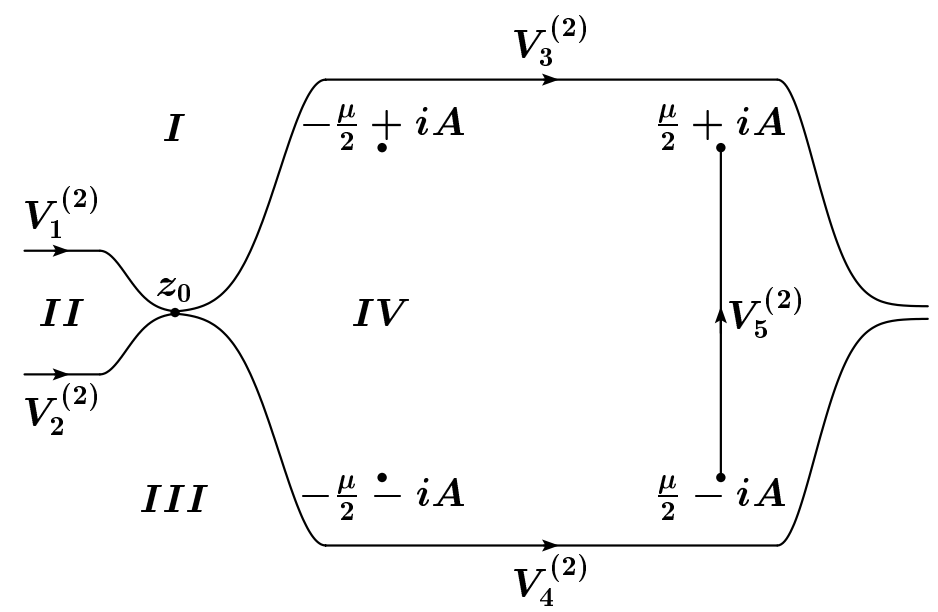

FIGURE 2.5. The RHP $P^{(2)}$ for the plane-wave region. Regions I through IV are used in the definition of $M^{(3)}$.

with

$$
\begin{gathered}
V_{1}^{(3)}=\left[\begin{array}{cc}
1 & \frac{\delta^{2} \rho e^{-i f t}}{1+\rho^{2}} \\
0 & 1
\end{array}\right], \quad V_{2}^{(3)}=\left[\begin{array}{cc}
1 & 0 \\
\frac{\delta^{-2} \rho e^{i f t}}{1+\rho^{2}} & 1
\end{array}\right], \quad V_{3}^{(3)}=\left[\begin{array}{cc}
1 & 0 \\
\delta^{-2} \rho e^{i f t} & 1
\end{array}\right], \\
V_{4}^{(3)}=\left[\begin{array}{cc}
1 & \delta^{2} \rho e^{-i f t} \\
0 & 1
\end{array}\right], \quad V_{5}^{(3)}=\left[\begin{array}{cc}
0 & \delta^{2} \\
-\delta^{-2} & 0
\end{array}\right] .
\end{gathered}
$$

\section{6 $P^{(3)} \rightarrow P^{(4)}$ : Removal of $\delta(z)$ from the Jump on the $\lambda_{R}$ Cut}

A $g$-function $[7,10]$ is introduced to remove the $\delta$ terms from $V_{5}^{(3)}$. Define

$$
M^{(4)}=M^{(3)} e^{i g(z) \sigma_{3}} .
$$

Here $g(z)$ is a to-be-determined function that is analytic off the $\lambda_{R}$ branch cut. On the $\lambda_{R}$ cut, the new jump matrix is given by

$$
\begin{aligned}
V_{5}^{(4)} & =e^{-i g_{-} \sigma_{3}} V_{5}^{(3)} e^{i g_{+} \sigma_{3}} \\
& =\left[\begin{array}{cc}
0 & \delta^{2} e^{-i\left(g_{+}+g_{-}\right)} \\
-\delta^{-2} e^{i\left(g_{+}+g_{-}\right)} & 0
\end{array}\right] .
\end{aligned}
$$

Recalling the explicit formula for $\delta(z), V_{5}^{(4)}$ will be a constant matrix if

$$
g_{+}+g_{-}+\frac{1}{\pi} \int_{-\infty}^{z_{0}} \frac{\ln \left(1+\rho^{2}(\zeta)\right)}{\zeta-z} \mathrm{~d} \zeta=\omega_{R} \quad \text { on the } \lambda_{R} \text { branch cut }
$$

for some real constant $\omega_{R}$. Take $\omega_{R}=0$. Now equation (2.20) is a scalar RHP for $g$. Divide by $\lambda_{R}$, remembering that $\lambda_{R}=\lambda_{R-}=-\lambda_{R+}$ on the branch cut:

$$
\frac{g_{+}}{\lambda_{R-}}+\frac{g_{-}}{\lambda_{R-}}=-\frac{1}{\lambda_{R-} \pi} \int_{-\infty}^{z_{0}} \frac{\ln \left(1+\rho^{2}(\zeta)\right)}{\zeta-z} \mathrm{~d} \zeta,
$$


so

$$
\left(\frac{g}{\lambda_{R}}\right)_{+}-\left(\frac{g}{\lambda_{R}}\right)_{-}=\frac{1}{\lambda_{R-} \pi} \int_{-\infty}^{z_{0}} \frac{\ln \left(1+\rho^{2}(\zeta)\right)}{\zeta-z} \mathrm{~d} \zeta .
$$

Therefore, by the Plemelj formula (see Deift [4, sec. 1.1]),

$$
g(z)=\frac{\lambda_{R}(z)}{2 \pi^{2} i} \int_{\lambda_{R} \text { cut }} \frac{1}{(\eta-z) \lambda_{R-}(\eta)} \int_{-\infty}^{z_{0}} \frac{\ln \left(1+\rho^{2}(\zeta)\right)}{\zeta-\eta} \mathrm{d} \zeta \mathrm{d} \eta
$$

As $z \rightarrow \infty$, using $\lambda_{R}=z+O(1)$,

$$
\begin{aligned}
g(z) & =-\frac{\lambda_{R}(z)}{2 \pi^{2} i z} \int_{\lambda_{R} \text { cut }} \frac{1}{(1-\eta / z) \lambda_{R-}(\eta)} \int_{-\infty}^{z_{0}} \frac{\ln \left(1+\rho^{2}(\zeta)\right)}{\zeta-\eta} \mathrm{d} \zeta \mathrm{d} \eta \\
& =-\frac{\lambda_{R}(z)}{2 \pi^{2} i z} \int_{\lambda_{R} \text { cut }} \frac{1}{\lambda_{R-}(\eta)} \int_{-\infty}^{z_{0}} \frac{\ln \left(1+\rho^{2}(\zeta)\right)}{\zeta-\eta} \mathrm{d} \zeta\left(1+\frac{\eta}{z}+\frac{\eta^{2}}{z^{2}}+\cdots\right) \mathrm{d} \eta \\
& =-\frac{1}{2 \pi^{2} i} \int_{\lambda_{R} \text { cut }} \frac{1}{\lambda_{R-}(\eta)} \int_{-\infty}^{z_{0}} \frac{\ln \left(1+\rho^{2}(\zeta)\right)}{\zeta-\eta} \mathrm{d} \zeta \mathrm{d} \eta+O\left(z^{-1}\right) .
\end{aligned}
$$

Therefore, $g(z) \sim g(\infty)$ as $z \rightarrow \infty$, where

$$
g(\infty)=-\frac{1}{2 \pi^{2} i} \int_{\lambda_{R} \text { cut }} \frac{1}{\lambda_{R-}(\eta)} \int_{-\infty}^{z_{0}} \frac{\ln \left(1+\rho^{2}(\zeta)\right)}{\zeta-\eta} \mathrm{d} \zeta \mathrm{d} \eta
$$

depends on $\xi, A$, and $\mu$, but is independent of $z$. Now $M^{(4)}$ is the solution to the RHP

$$
P^{(4)}:\left\{\Sigma^{(4)}=\Sigma^{(3)}, V^{(4)}, e^{i g(\infty) \sigma_{3}} \text { as } z \rightarrow \infty\right\},
$$

where

$$
\begin{aligned}
& V_{1}^{(4)}=\left[\begin{array}{cc}
1 & \frac{\delta^{2} \rho e^{-i(f t+2 g)}}{1+\rho^{2}} \\
0 & 1
\end{array}\right], \quad V_{2}^{(4)}=\left[\begin{array}{cc}
1 & 0 \\
\frac{\delta^{-2} \rho e^{i(f t+2 g)}}{1+\rho^{2}} & 1
\end{array}\right], \\
& V_{3}^{(4)}=\left[\begin{array}{cc}
1 & 0 \\
\delta^{-2} \rho e^{i(f t+2 g)} & 1
\end{array}\right], \quad V_{4}^{(4)}=\left[\begin{array}{cc}
1 & \delta^{2} \rho e^{-i(f t+2 g)} \\
0 & 1
\end{array}\right], \\
& V_{5}^{(4)}=\left[\begin{array}{ll}
0 & 1 \\
-1 & 0
\end{array}\right] .
\end{aligned}
$$

\subsection{Model Problem $\boldsymbol{P}^{(\bmod )}$}

The jump matrices $V_{1}^{(4)}, V_{2}^{(4)}, V_{3}^{(4)}$, and $V_{4}^{(4)}$ decay exponentially to the identity away from the point $z_{0}$ as $t \rightarrow \infty$. Disregarding the jump on these contours leaves the jump $V_{5}^{(4)}$ on the $\lambda_{R}$ branch cut. We prove below that this problem, dubbed the model problem, will produce the leading-order solution. The other contours 


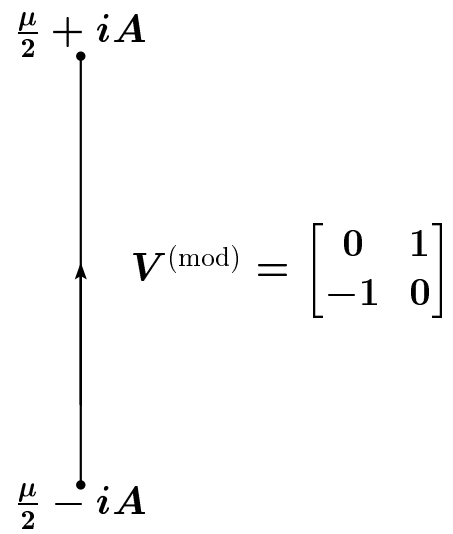

FIGURE 2.6. The RHP $P^{(\mathrm{mod})}$ for the plane-wave region.

provide higher-order corrections. $M^{(\mathrm{mod})}$ (see Figure 2.6) is the solution to the RHP

$$
P^{(\mathrm{mod})}:\left\{\Sigma^{(\mathrm{mod})}=\lambda_{R} \text { cut, } V^{(\mathrm{mod})}, e^{i g(\infty) \sigma_{3}} \text { as } z \rightarrow \infty\right\},
$$

with

$$
V^{(\mathrm{mod})}=V_{5}^{(4)}=\left[\begin{array}{rr}
0 & 1 \\
-1 & 0
\end{array}\right]
$$

For large $z$, we introduce the factorization $M^{(3)}=M^{(\mathrm{err})} M^{(\mathrm{mod})}$, where the higher-order contribution from the contours besides the $\lambda_{R}$ branch cut have been factored out into an error term. We write the Laurent series for a matrix $M$ as

$$
M=M_{0}+\frac{M_{1}}{z}+\frac{M_{2}}{z^{2}}+\cdots \quad \text { as } z \rightarrow \infty .
$$

Then $\left(M_{1}^{(3)}\right)_{12}=\left(M_{1}^{(\mathrm{mod})}+M_{1}^{(\mathrm{err})}\right)_{12}$, where $(M)_{12}$ is the 12-entry of $M$. Therefore, by equation (A.40),

$$
q(x, t)=-2\left(M_{1}^{(\mathrm{mod})}+M_{1}^{(\mathrm{err})}\right)_{12} e^{-i\left[\mu|x|+\left(\mu^{2} / 2-A^{2}\right) t-g(\infty)\right]} .
$$

By representing $M^{(\mathrm{err})}$ as the solution to a RHP, we show in the following sections:

LEMMA $2.1\left|M_{1}^{(\mathrm{err})}\right|=O\left(t^{-1 / 2}\right)$.

Furthermore, the model RHP is solved explicitly by

$$
M^{(\bmod )}=\left[\begin{array}{cc}
e^{i g(\infty)} & 0 \\
0 & e^{-i g(\infty)}
\end{array}\right]\left[\begin{array}{cc}
\frac{L+L^{-1}}{2} & \frac{-i L+i L^{-1}}{2} \\
\frac{i L-i L^{-1}}{2} & \frac{L+L^{-1}}{2}
\end{array}\right],
$$

where

$$
L(z)=\left(\frac{z-\mu / 2-i A}{z-\mu / 2+i A}\right)^{1 / 4}
$$


The sheet is chosen so $L \rightarrow 1$ as $z \rightarrow \infty$. This formula for $M^{(\text {mod) }}$ gives

$$
\left(M_{1}^{(\bmod )}\right)_{12}=-\frac{A}{2} e^{i g(\infty)} .
$$

Equations (2.28) and (2.31) along with Lemma 2.1 complete the proof of Theorem 1.1. Note that in the absence of the shock, the wave would have the form $A e^{-i\left(\mu|\xi|+\mu^{2} / 2-A^{2}\right) t}$. Therefore the effect of the shock is to impart an $O(1)$ shift $2 i g(\infty)$ to the $O(t)$ phase. From equation (2.25), this phase shift is nonzero but decaying to 0 as $\xi \rightarrow \infty$.

\subsection{The Error Problem $\boldsymbol{P}^{(\mathrm{err})}$}

It would be expedient to extend the factorization $M^{(3)}=M^{(\mathrm{err})} M^{(\mathrm{mod})}$ to the entire complex plane. However, the decay on the other contours is not uniform in $z$ near $z_{0}$, a condition that is necessary for the error estimate. Therefore the strategy is to write

$$
M^{(3)}=M^{(\mathrm{err})} M^{(\mathrm{app})},
$$

where $M^{(\text {app })}$ includes the jump across the $\lambda_{R}$ branch cut and near $z_{0}$.

For a given $R \in \mathbb{R}$ and $C \in \mathbb{C}$, let $r_{C}^{R}\left(D_{C}^{R}\right)$ be the circle (closed disk) of radius $R$ centered at $z=C$. Choose $\varepsilon$ sufficiently small so that $r_{z_{0}}^{\varepsilon}$ does not intersect the $\lambda_{L}$ branch cut and define

$$
M^{(\mathrm{app})}= \begin{cases}\text { parametrix of } M^{(3)} & \text { inside } r_{z_{0}}^{\varepsilon}, \\ M^{(\mathrm{mod})} & \text { outside } r_{z_{0}}^{\varepsilon}\end{cases}
$$

By a parametrix of $M^{(3)}$ we mean $M^{(\mathrm{app})}$ satisfies the same jump conditions as $M^{(3)}$ inside $r_{z_{0}}^{\varepsilon}$. $M^{(\text {app })}$ will have a jump $V_{z_{0}}^{(\text {app) }}$ across the circle $r_{z_{0}}^{\varepsilon}$. The construction of the parametrix is deferred until Appendix B. There $V_{z_{0}}^{(\text {app })}$ is shown to have the form $I+O\left(t^{-1 / 2}\right)$, which suffices for the error estimate. $r_{z_{0}}^{\varepsilon}$ is oriented counterclockwise. $M^{(\text {app })}$ satisfies the RHP

$$
P^{(\text {app })}:\left\{\Sigma^{(\text {app })} \text { (see Figure 2.7), } V^{(\text {app })}, e^{i g(\infty) \sigma_{3}} \text { as } z \rightarrow \infty\right\}
$$

with

$$
\begin{gathered}
V_{i}^{(\text {app })}=V_{i}^{(3)} \text { inside } r_{z_{0}}^{\varepsilon}, \quad i=1,2,3,4, \\
V_{R}^{(\text {app })}=V^{(\text {mod })}, \\
V_{z_{0}}^{(\text {app })}=I+O\left(t^{-1 / 2}\right) \quad \text { on } r_{z_{0}}^{\varepsilon} .
\end{gathered}
$$

The definition of $M^{(\mathrm{app})}$ and equation (2.32) constrain $M^{(\mathrm{err})}$ to satisfy the RHP

$$
P^{(\mathrm{err})}:\left\{\Sigma^{(\mathrm{err})} \text { (see Figure 2.8), } V^{(\mathrm{err})}, I \text { as } z \rightarrow \infty\right\},
$$

with $\Sigma^{(\text {err })}$ and $V^{(\text {err) }}$ defined by Figure 2.8 and

$$
\begin{array}{ll}
V_{i}^{(\mathrm{err})}=M^{(\mathrm{app})} V_{i}^{(3)}\left(M^{(\mathrm{app})}\right)^{-1} & \text { outside } r_{z_{0}}^{\varepsilon}, i=1,2,3,4, \\
V_{z_{0}}^{(\mathrm{err})}=M_{-}^{(\mathrm{app})}\left(V_{z_{0}}^{(\mathrm{app})}\right)^{-1}\left(M_{-}^{(\mathrm{app})}\right)^{-1} & \text { on } r_{z_{0}}^{\varepsilon} .
\end{array}
$$



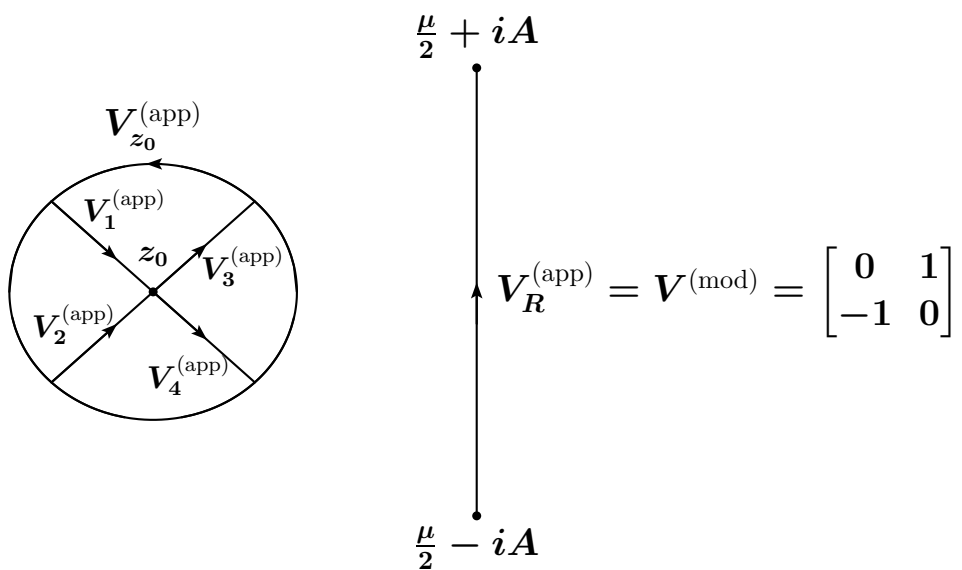

FIGURE 2.7. The RHP $P^{(\mathrm{app})}$ for the plane-wave region. The circle $r_{z_{0}}^{\varepsilon}$ is enlarged to show detail.

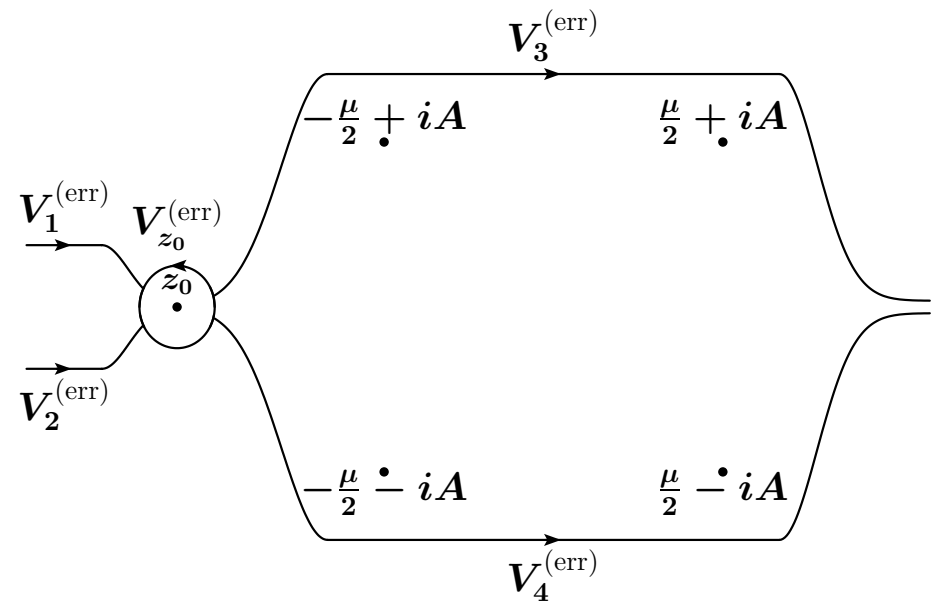

FIGURE 2.8. The RHP $P^{(\mathrm{err})}$ for the plane-wave region.

\subsection{Error Bound on $M_{1}^{(\mathrm{err})}$}

We now prove Lemma 2.1. Given an oriented contour $\Sigma$, define the Cauchy transform $C_{\Sigma}$ as

$$
\left(C_{\Sigma}(f)\right)(z)=\frac{1}{2 \pi i} \int_{\Sigma} \frac{f(\zeta)}{(\zeta-z)} d \zeta
$$


Define $C_{\Sigma}^{+}(f)$ and $C_{\Sigma}^{-}(f)$ to be the nontangential limits of $C_{\Sigma}(f)$ approaching $\Sigma$ from the left and right, respectively. Also, given a matrix $V$ defined on $\Sigma$, let

$$
C_{V}^{-} f=C_{\Sigma}^{-}(f(V-I)) \text {. }
$$

We have the identity

$$
\begin{aligned}
M^{(\mathrm{err})}-I & =C_{\Sigma^{(\mathrm{err})}} M_{-}^{(\mathrm{err})}\left(V^{(\mathrm{err})}-I\right) \\
& =\frac{1}{2 \pi i} \int_{\Sigma^{(\mathrm{err})}} \frac{M_{-}^{(\mathrm{err})}(\zeta)\left(V^{(\mathrm{err})}(\zeta)-I\right)}{\zeta-z} \mathrm{~d} \zeta \\
& =-\frac{1}{2 \pi i z} \int_{\Sigma^{(\mathrm{err})}} M_{-}^{(\mathrm{err})}(\zeta)\left(V^{(\mathrm{err})}(\zeta)-I\right) \mathrm{d} \zeta+O\left(z^{-2}\right) .
\end{aligned}
$$

Therefore, since $M_{1}^{(\mathrm{err})}=\lim _{z \rightarrow \infty} z\left(M^{(\mathrm{err})}-I\right)$,

$$
M_{1}^{(\mathrm{err})}=-\frac{1}{2 \pi i} \int_{\Sigma^{(\mathrm{err})}} M_{-}^{(\mathrm{err})}(\zeta)\left(V^{(\mathrm{err})}(\zeta)-I\right) \mathrm{d} \zeta .
$$

Thus,

$$
\left|M_{1}^{(\mathrm{err})}\right| \leq C_{1}\left\|M_{-}^{(\mathrm{err})}-I\right\|_{L^{2}}\left\|V^{(\mathrm{err})}-I\right\|_{L^{2}}+C_{2}\left\|V^{(\mathrm{err})}-I\right\|_{L^{1}}
$$

for positive constants $C_{1}$ and $C_{2}$. On $\Sigma^{(\text {err })} \backslash r_{z 0}^{\varepsilon}, V^{(\text {err })}$ decays uniformly in $t$, so there exist positive constants $C_{3}$ and $C_{4}$ such that

$$
\left\|V^{(\mathrm{err})}-I\right\|_{L^{p}\left(\Sigma^{(\mathrm{err})} \backslash r_{z_{0}}^{\varepsilon}\right)} \leq C_{3} e^{-C_{4} t}, \quad p=1,2
$$

From equation (B.45) in Appendix B,

$$
\left\|V^{(\mathrm{err})}-I\right\|_{L^{p}\left(r_{z_{0}}^{\varepsilon}\right)}=O\left(t^{-1 / 2}\right), \quad p=1,2 .
$$

From equations (2.41) and (2.42),

$$
\left\|V^{(\mathrm{err})}-I\right\|_{L^{p}\left(\Sigma^{(\mathrm{err})}\right)}=O\left(t^{-1 / 2}\right), \quad p=1,2 .
$$

We also have the identity

$$
M_{-}^{(\mathrm{err})}-I=\left(I-C_{V^{(\mathrm{err})}}^{-}\right)^{-1} C_{V^{(\mathrm{err})}}^{-} I,
$$

so

$$
\begin{aligned}
\left\|M_{-}^{(\mathrm{err})}-I\right\|_{L^{2}\left(\Sigma^{(\mathrm{err})}\right)} & \leq\left\|\left(I-C_{V^{(\mathrm{err})}}^{-}\right)^{-1}\right\| \cdot\left\|C_{V^{(\mathrm{err})}}^{-} I\right\|_{L^{2}\left(\Sigma^{(\mathrm{err})}\right)} \\
& \leq C\left\|V^{(\mathrm{err})}-I\right\|_{L^{2}\left(\Sigma^{(\mathrm{err})}\right)} \\
& =O\left(t^{-1 / 2}\right)
\end{aligned}
$$

for some constant $C$, since $V^{(\text {err })}$ is uniformly close to $I$ on $\Sigma^{(\text {err })}$ as $t \rightarrow \infty$. Together, (2.40), (2.43), and (2.45) give $\left|M_{1}^{\text {(err) }}\right|=O\left(t^{-1 / 2}\right)$, which completes the proof of Lemma 2.1 and thus Theorem 1.1. 


\section{Solution in the Residual Region}

For $0<\xi<\xi_{2}, \Im(f)$ is negative along part or all of the $\lambda_{L}$ branch cut. Therefore the method used in the initial region will not work. A new factorization of the jump on the $\lambda_{L}$ cut leads to a two-banded model problem that is solved using theta functions.

To avoid overly cumbersome notation, some symbols will be reused in this section and the next. For instance, the sequence of RHPs will again be labeled $P^{(i)}$, even though $P^{(i)}$ may be different in different regions. Notation is not reused in a single section, and the exact meaning will be clear from context.

\subsection{Overview of the Solution in the Residual Region}

We start with the RHP $P^{(0)}$ of Figure 1.4 and perform a new set of transformations.

- $P^{(0)} \rightarrow P^{(1)}$ : We define the new RHP $P^{(1)}$ in Section 3.2 by introducing a new factorization on the $\lambda_{L}$ branch cut. The point $z_{0}$ is now to the right of $-\mu / 2$.

- $P^{(1)} \rightarrow P^{(2)}$ : The jump across $\left(-\infty, z_{0}\right)$ is removed by $P^{(2)}$ in Section 3.3.

- $P^{(2)} \rightarrow P^{(3)}$ : The factor $D(z)$ is removed from the jumps by the definition of $P^{(3)}$ in Section 3.4.

- $P^{(3)} \rightarrow P^{(4)}$ : The point $z_{0}$ and the RHP $P^{(4)}$ are defined in Section 3.5 by introducing the function $G(z)$, which removes the exponential blowup of the jump on the $\lambda_{L}$ cut.

- $P^{(4)} \rightarrow P^{(5)}$ : The jumps on the branch cuts are reduced to constants using the function $g(z)$ by the definition of $P^{(5)}$ in Section 3.7.

The two-banded model problem $P^{(\bmod )}$ is defined and solved explicitly in terms of theta functions in Section 3.8. The approximate and error problems $P^{(\text {app) }}$ and $P^{\text {(err) }}$ are defined in Section 3.9. The error estimate follows that of the plane-wave region, with an overview provided in Section 3.10.

This new method will work for $0<\xi \leq \xi_{1}$ but breaks down for $\xi_{1}<\xi<\xi_{2}$ for some $\xi_{1}$ depending on $A$ and $\mu$. Why and when this breakdown occurs is discussed in Section 3.6. The transition region $\xi_{1}<\xi<\xi_{2}$ will be covered in Section 4. For now assume $0<\xi<\xi_{1}$.

\section{2 $P^{(0)} \rightarrow P^{(1)}$ : Factorization of $V^{(0)}$ and Deformation of $\Sigma^{(0)}$}

Start with the RHP $P^{(0)}$ from Section 1.5. Define

$$
\begin{array}{rlrl}
V_{1}^{(1)} & =\left[\begin{array}{cc}
\frac{1}{D^{1 / 2}} & \frac{D^{1 / 2} \rho}{1+\rho^{2}} e^{-i f t} \\
0 & D^{1 / 2}
\end{array}\right], & V_{2}^{(1)}=\left[\begin{array}{cc}
\frac{1}{D^{1 / 2}} & 0 \\
\frac{D^{1 / 2} \rho}{1+\rho^{2}} e^{i f t} & D^{1 / 2}
\end{array}\right], \\
V_{3}^{(1)}=\left[\begin{array}{cc}
\frac{1}{D^{1 / 2}} & 0 \\
\frac{\rho}{D^{1 / 2}} e^{i f t} & D^{1 / 2}
\end{array}\right], & V_{4}^{(1)}=\left[\begin{array}{cc}
\frac{1}{D^{1 / 2}} & \frac{\rho}{D^{1 / 2}} e^{-i f t} \\
0 & D^{1 / 2}
\end{array}\right],
\end{array}
$$




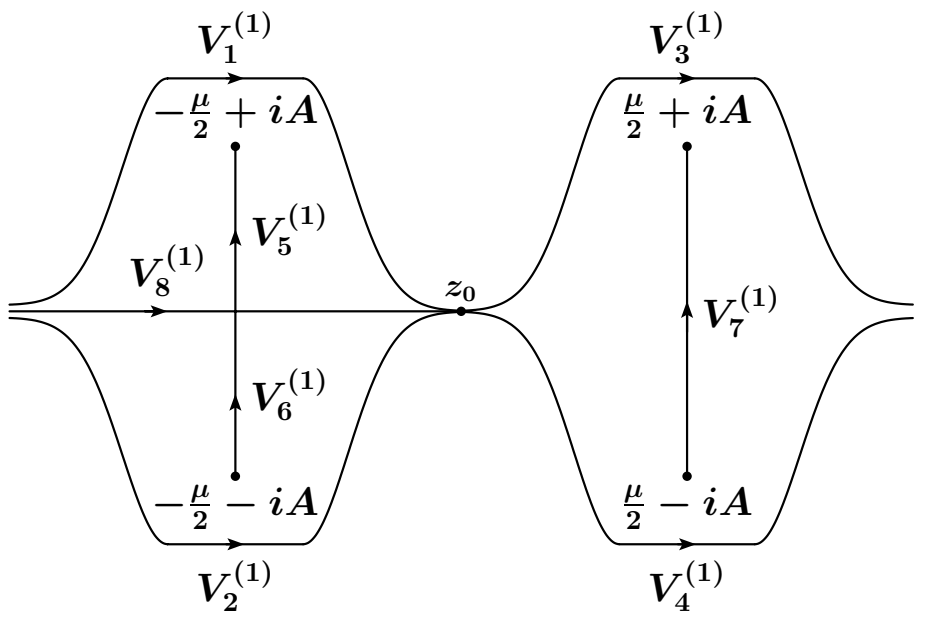

FIGURE 3.1. The RHP $P^{(1)}$ for the residual region.

$$
\begin{array}{rlrl}
V_{5}^{(1)} & =\left[\begin{array}{cc}
0 & \frac{\rho_{-}}{1+\rho_{-}^{2}} e^{-i f t} \\
-\frac{1+\rho_{-}^{2}}{\rho_{-}} e^{i f t} & 0
\end{array}\right], & V_{6}^{(1)} & =\left[\begin{array}{cc}
0 & \frac{1+\rho_{-}^{2}}{\rho_{-}} e^{-i f t} \\
\frac{-\rho_{-}}{1+\rho_{-}^{2}} e^{i f t} & 0
\end{array}\right], \\
V_{7}^{(1)}=\left[\begin{array}{rr}
0 & 1 \\
-1 & 0
\end{array}\right], & V_{8}^{(1)}=\left[\begin{array}{cc}
1+\rho^{2} & 0 \\
0 & \frac{1}{1+\rho^{2}}
\end{array}\right] .
\end{array}
$$

The factorizations used are similar to those used before except on the $\lambda_{L}$ branch cut. Using $\rho_{+}=-1 / \rho_{-}$on the $\lambda_{L}$ branch cut, we factor

$$
\begin{array}{ll}
V_{1}^{(0)}=\left(V_{1-}^{(1)}\right)^{-1} V_{5}^{(1)} V_{1+}^{(1)} & \text { on the } \lambda_{L} \text { cut in the upper half-plane, } \\
V_{2}^{(0)}=V_{2-}^{(1)} V_{6}^{(1)}\left(V_{2+}^{(1)}\right)^{-1} & \text { on the } \lambda_{L} \text { cut in the lower half-plane, } \\
V_{3}^{(0)}=\left(V_{3-}^{(1)}\right)^{-1} V_{7}^{(1)} V_{3+}^{(1)} & \text { on the } \lambda_{R} \text { cut in the upper half-plane, } \\
V_{4}^{(0)}=V_{4-}^{(1)} V_{7}^{(1)}\left(V_{4+}^{(1)}\right)^{-1} & \text { on the } \lambda_{R} \text { cut in the lower half-plane, } \\
V_{5}^{(0)}=V_{2}^{(1)} V_{8}^{(1)} V_{1}^{(1)} & \text { on }\left(-\infty, z_{0}\right), \\
V_{5}^{(0)}=V_{4}^{(1)} V_{3}^{(1)} & \text { on }\left(z_{0}, \infty\right) .
\end{array}
$$

Assuming $-\frac{\mu}{2}<z_{0}<\frac{\mu}{2}$, splitting and deforming $\Sigma^{(0)}$ in a similar manner as in Section 2.3 gives the RHP

$$
P^{(1)}:\left\{\Sigma^{(1)}, V^{(1)}, I \text { as } z \rightarrow \infty\right\},
$$

where $\Sigma^{(1)}$ and $V^{(1)}$ are defined in Figure 3.1 and equations (3.1). As in the planewave region, two different deformations are used along the real axis. The point at which the factorization changes is again called $z_{0}$. However, $z_{0}$ will not necessarily be a point where $\Im(f)$ changes sign. Instead it will be where the imaginary part of a new function $h(z)$ changes sign. $h$ and $z_{0}$ are found in Section 3.5. 


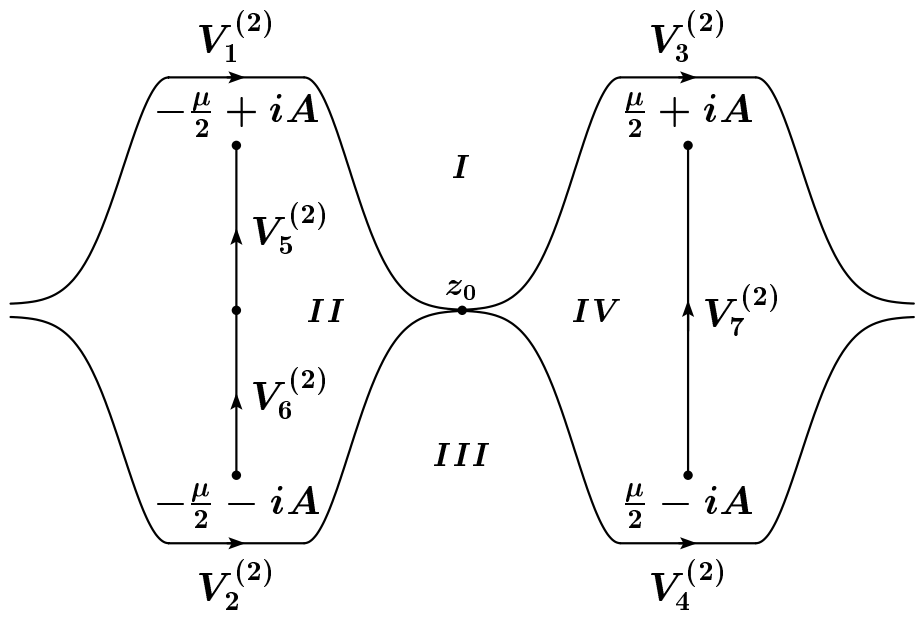

FIGURE 3.2. The RHP $P^{(2)}$ for the residual region. The regions I through IV are used in the definition of $M^{(3)}$.

\section{3 $P^{(1)} \rightarrow P^{(2)}$ : Elimination of the Jump on $\left(-\infty, z_{0}\right)$}

The contour on the real axis with jump matrix $V_{8}^{(1)}$ can be removed using a $\delta$-function as in the plane-wave region (see Section 2.4). The function

$$
\delta(z)=\exp \left(\frac{1}{2 \pi i} \int_{-\infty}^{z_{0}} \frac{\ln \left(1+[\rho(\zeta)]^{2}\right)}{\zeta-z} \mathrm{~d} \zeta\right)
$$

satisfies the appropriate RHP. Define $M^{(2)}=M^{(1)} \delta^{-\sigma_{3}} \cdot M^{(2)}$ satisfies the RHP

$$
P^{(2)}:\left\{\Sigma^{(2)}=\Sigma^{(1)} \backslash \Sigma^{(\delta)}, V^{(2)}, I \text { as } z \rightarrow \infty\right\} .
$$

$V^{(2)}$ is defined by Figure 3.2 and

$$
\begin{array}{ll}
V_{1}^{(2)}=\left[\begin{array}{cc}
\frac{1}{D^{1 / 2}} & \delta^{2} D^{1 / 2} P^{-1} e^{-i f t} \\
0 & D^{1 / 2}
\end{array}\right], & V_{2}^{(2)}=\left[\begin{array}{cc}
\frac{1}{D^{1 / 2}} & 0 \\
\delta^{-2} D^{1 / 2} P^{-1} e^{i f t} & D^{1 / 2}
\end{array}\right], \\
V_{3}^{(2)}=\left[\begin{array}{cc}
\frac{1}{D^{1 / 2}} & 0 \\
\delta^{-2} \frac{\rho}{D^{1 / 2}} e^{i f t} & D^{1 / 2}
\end{array}\right], & V_{4}^{(2)}=\left[\begin{array}{cc}
\frac{1}{D^{1 / 2}} & \delta^{2} \frac{\rho}{D^{1 / 2}} e^{-i f t} \\
0 & D^{1 / 2}
\end{array}\right], \\
V_{5}^{(2)}=\left[\begin{array}{cc}
0 & \delta^{2} P_{-}^{-1} e^{-i f t} \\
-\delta^{-2} P_{-} e^{i f t} & 0
\end{array}\right], & V_{6}^{(2)}=\left[\begin{array}{cc}
0 & \delta^{2} P_{-} e^{-i f t} \\
-\delta^{-2} P_{-}^{-1} e^{i f t} & 0
\end{array}\right], \\
V_{7}^{(2)}=\left[\begin{array}{cc}
0 & \delta^{2} \\
-\delta^{-2} & 0
\end{array}\right], &
\end{array}
$$

wherein

$$
P(z)=\frac{1+\rho^{2}}{\rho}
$$




\section{$3.4 P^{(2)} \rightarrow P^{(3)}$ : Removal of $D(z)$}

The factor $D(z)$ is removed from the jump matrices in the same manner as in the plane-wave region. Define $M^{(3)}$ by

$$
M^{(3)}= \begin{cases}M^{(2)} D^{\sigma_{3} / 2} & \text { in region I, } \\ M^{(2)} D^{-\sigma_{3} / 2} & \text { in region III, } \\ M^{(2)} & \text { in regions II and IV. }\end{cases}
$$

Regions I through IV are defined in Figure 3.2. The RHP satisfied by $M^{(3)}$ is

$$
P^{(3)}:\left\{\Sigma^{(3)}=\Sigma^{(2)}, V^{(3)}, I \text { as } z \rightarrow \infty\right\}
$$

with

$$
\begin{array}{rlrl}
V_{1}^{(3)} & =\left[\begin{array}{cc}
1 & \delta^{2} P^{-1} e^{-i f t} \\
0 & 1
\end{array}\right], & V_{2}^{(2)}=\left[\begin{array}{cc}
1 & 0 \\
\delta^{-2} P^{-1} e^{i f t} & 1
\end{array}\right], \\
V_{3}^{(3)}=\left[\begin{array}{cc}
1 & 0 \\
\delta^{-2} \rho e^{i f t} & 1
\end{array}\right], & V_{4}^{(3)}=\left[\begin{array}{cc}
1 & \delta^{2} \rho e^{-i f t} \\
0 & 1
\end{array}\right], \\
V_{5}^{(3)}=\left[\begin{array}{cc}
0 & \delta^{2} P_{-}^{-1} e^{-i f t} \\
-\delta^{-2} P_{-} e^{i f t} & 0
\end{array}\right], & V_{6}^{(3)}=\left[\begin{array}{cc}
0 & \delta^{2} P_{-} e^{-i f t} \\
-\delta^{-2} P_{-}^{-1} e^{i f t} & 0
\end{array}\right], \\
V_{7}^{(3)}=\left[\begin{array}{cc}
0 & \delta^{2} \\
-\delta^{-2} & 0
\end{array}\right] . &
\end{array}
$$

\section{5 $P^{(3)} \rightarrow P^{(4)}$ : The $g$-Function Mechanism}

Although the jump on the $\lambda_{L}$ cut has the desired off-diagonal form, the terms involving $e^{i f t}$ in $V_{5}^{(3)}$ and $e^{-i f t}$ in $V_{6}^{(3)}$ are still increasing exponentially in time. A $g$-function is now introduced to eliminate this growth.

Define

$$
M^{(4)}=M^{(3)} e^{i G \sigma_{3} t}
$$

Here $G(z)$ is a to-be-determined scalar function that is analytic everywhere except on the $\lambda_{L}$ and $\lambda_{R}$ branch cuts. $M^{(4)}$ satisfies the RHP

$$
P^{(4)}:\left\{\Sigma^{(4)}=\Sigma^{(3)}, V^{(4)}, e^{i G(\infty) \sigma_{3} t} \text { as } z \rightarrow \infty\right\},
$$

with

$$
V^{(4)}=e^{-i G_{-} \sigma_{3} t} V^{(3)} e^{i G_{+} \sigma_{3} t} .
$$


Explicitly,

$$
\begin{aligned}
& V_{1}^{(4)}=\left[\begin{array}{cc}
1 & \delta^{2} P^{-1} e^{-i(f+2 G) t} \\
0 & 1
\end{array}\right], \quad V_{2}^{(4)}=\left[\begin{array}{cc}
1 & 0 \\
\delta^{-2} P^{-1} e^{i(f+2 G) t} & 1
\end{array}\right], \\
& V_{3}^{(4)}=\left[\begin{array}{cc}
1 & 0 \\
\delta^{-2} \rho e^{i(f+2 G) t} & 1
\end{array}\right], \quad V_{4}^{(4)}=\left[\begin{array}{cc}
1 & \delta^{2} \rho e^{-i(f+2 G) t} \\
0 & 1
\end{array}\right], \\
& V_{5}^{(4)}=\left[\begin{array}{cc}
0 & \delta^{2} P_{-}^{-1} e^{-i\left(f+G_{+}+G_{-}\right) t} \\
-\delta^{-2} P_{-} e^{i\left(f+G_{+}+G_{-}\right) t} & 0
\end{array}\right], \\
& V_{6}^{(4)}=\left[\begin{array}{cc}
0 & \delta^{2} P_{-} e^{-i\left(f+G_{+}+G_{-}\right) t} \\
-\delta^{-2} P_{-}^{-1} e^{i\left(f+G_{+}+G_{-}\right) t} & 0
\end{array}\right], \\
& V_{7}^{(4)}=\left[\begin{array}{cc}
0 & \delta^{2} e^{-i\left(G_{+}+G_{-}\right) t} \\
-\delta^{-2} e^{i\left(G_{+}+G_{-}\right) t} & 0
\end{array}\right]
\end{aligned}
$$

To remove the growth in time from $V_{5}^{(4)}$ and $V_{6}^{(4)}, f+G_{+}+G_{-}$should be a real constant along the $\lambda_{L}$ branch cut. To avoid introducing blowup in $V_{7}^{(4)}, G_{+}+G_{-}$ should be a real constant on the $\lambda_{R}$ cut. This constant is normalized to be 0 . This normalization changes the value of $G(\infty)$ but has no other effect. Therefore $G(z)$ satisfies the scalar RHP

- $G$ is analytic off the $\lambda_{L}$ and $\lambda_{R}$ branch cuts,

- $\begin{cases}f+G_{+}+G_{-}=\Omega_{L} & \text { on the } \lambda_{L} \text { branch cut for some real constant } \Omega_{L}, \\ G_{+}+G_{-}=0 & \text { on the } \lambda_{R} \text { branch cut, }\end{cases}$

- $G=O(1)$ as $z \rightarrow \infty$.

To deal with the condition $G_{+}+G_{-}=0$ on the $\lambda_{R}$ branch cut, let

$$
G(z)=\lambda_{R}(z) k(z),
$$

where $k(z)$ is analytic off the $\lambda_{L}$ branch cut. Then

$$
G_{+}+G_{-}=\left(\lambda_{R} k\right)_{+}+\left(\lambda_{R} k\right)_{-}=\lambda_{R+} k-\lambda_{R+} k=0 .
$$

$k(z)$ satisfies the scalar jump condition

$$
k_{+}+k_{-}=\frac{-f+\Omega_{L}}{\lambda_{R}} \text { on the } \lambda_{L} \text { branch cut. }
$$

To change $k_{+}+k_{-}$to $k_{+}-k_{-}$, divide by $\lambda_{L_{-}}$:

$$
\frac{k_{+}}{\lambda_{L-}}+\frac{k_{-}}{\lambda_{L-}}=\frac{-f+\Omega_{L}}{\lambda_{L-} \lambda_{R}}
$$

or

$$
\left(\frac{k}{\lambda_{L}}\right)_{+}-\left(\frac{k}{\lambda_{L}}\right)_{-}=\frac{f-\Omega_{L}}{\lambda_{L-} \lambda_{R}}
$$


Therefore, by the Plemelj formula,

$$
k(z)=\frac{\lambda_{L}(z)}{2 \pi i} \int_{\lambda_{L} \text { cut }} \frac{f(\zeta)-\Omega_{L}}{\lambda_{L-}(\zeta) \lambda_{R}(\zeta)(\zeta-z)} \mathrm{d} \zeta,
$$

and so

$$
G(z)=\frac{\lambda_{L}(z) \lambda_{R}(z)}{2 \pi i} \int_{\lambda_{L} \text { cut }} \frac{f(\zeta)-\Omega_{L}}{\lambda_{L-}(\zeta) \lambda_{R}(\zeta)(\zeta-z)} \mathrm{d} \zeta .
$$

The constant $\Omega_{L}$ can be found by applying the condition $G=O(1)$ as $z \rightarrow \infty$. Indeed, using the fact that $\lambda_{L} \lambda_{R}=z^{2}+O(1)$, as $z \rightarrow \infty$

$$
\begin{aligned}
G(z)= & \frac{-\lambda_{L}(z) \lambda_{R}(z)}{2 \pi i z} \int_{\lambda_{L} \text { cut }} \frac{f(\zeta)-\Omega_{L}}{\lambda_{L-}(\zeta) \lambda_{R}(\zeta)\left(1-\frac{\zeta}{z}\right)} \mathrm{d} \zeta \\
= & \frac{-z^{2}+O(1)}{2 \pi i z} \int_{\lambda_{L} \text { cut }} \frac{f(\zeta)-\Omega_{L}}{\lambda_{L-}(\zeta) \lambda_{R}(\zeta)}\left(1+\frac{\zeta}{z}+O\left(\frac{1}{z^{2}}\right)\right) \mathrm{d} \zeta \\
= & \frac{-z}{2 \pi i} \int_{\lambda_{L} \text { cut }} \frac{f(\zeta)-\Omega_{L}}{\lambda_{L-}(\zeta) \lambda_{R}(\zeta)} \mathrm{d} \zeta \\
& -\frac{1}{2 \pi i} \int_{\lambda_{L} \text { cut }} \frac{f(\zeta)-\Omega_{L}}{\lambda_{L-}(\zeta) \lambda_{R}(\zeta)} \zeta \mathrm{d} \zeta+O\left(\frac{1}{z}\right) .
\end{aligned}
$$

For $G$ to approach a constant at infinity, it is necessary that

$$
\int_{\lambda_{L} \text { cut }} \frac{f(\zeta)-\Omega_{L}}{\lambda_{L-}(\zeta) \lambda_{R}(\zeta)} \mathrm{d} \zeta=0
$$

so

$$
\begin{aligned}
\Omega_{L} & =\left(\int_{\lambda_{L} \text { cut }} \frac{f(\zeta)}{\lambda_{L-}(\zeta) \lambda_{R}(\zeta)} \mathrm{d} \zeta\right) /\left(\int_{\lambda_{L} \text { cut }} \frac{1}{\lambda_{L-}(\zeta) \lambda_{R}(\zeta)} \mathrm{d} \zeta\right) \\
& =\left(\int_{-\frac{\mu}{2}-i A}^{-\frac{\mu}{2}+i A} \frac{2\left(\zeta+\frac{\mu}{2}+\xi\right)}{\left(A^{2}+\left(\zeta+\frac{\mu}{2}\right)^{2}\right)^{1 / 2}} \mathrm{~d} \zeta\right) /\left(\int_{\lambda_{L} \text { cut }} \frac{1}{\lambda_{L-}(\zeta) \lambda_{R}(\zeta)} \mathrm{d} \zeta\right) \\
& =2 \pi i \xi /\left(\int_{\lambda_{L} \text { cut }} \frac{1}{\lambda_{L-}(\zeta) \lambda_{R}(\zeta)} \mathrm{d} \zeta\right) .
\end{aligned}
$$

Furthermore,

$$
\lim _{z \rightarrow \infty} G(z)=G(\infty)=\frac{-1}{2 \pi i} \int_{\lambda_{L} \text { cut }} \frac{f(\zeta)-\Omega_{L}}{\lambda_{L-}(\zeta) \lambda_{R}(\zeta)} \zeta \mathrm{d} \zeta
$$


The last step in defining the RHP $P^{(4)}$ is to deform the contours with jump $V_{1}^{(4)}$ and $V_{4}^{(4)}$ into regions where $\Im(f+2 G)<0$, and the contours with jump $V_{2}^{(4)}$ and $V_{3}^{(4)}$ into regions where $\Im(f+2 G)>0$. This will not always be possible, in which case an alternate factorization is required; see Section 3.6. For now assume such a deformation exists. Define

$$
h(z)=f(z)+2 G(z) .
$$

In the residual region, there will be three points where the locus $\Im(h)=0$ intersects the real axis (see Figure 3.3(a), noting that $\pm \mu / 2$ are not along this locus). Define $z_{0}$ to be the middle of these three points. Using the conditions on $g, P^{(3)}$ is defined by

$$
\begin{aligned}
& V_{1}^{(4)}=\left[\begin{array}{cc}
1 & \delta^{2} P^{-1} e^{-i h t} \\
0 & 1
\end{array}\right], \quad V_{2}^{(3)}=\left[\begin{array}{cc}
1 & 0 \\
\delta^{-2} P^{-1} e^{i h t} & 1
\end{array}\right], \\
& V_{3}^{(4)}=\left[\begin{array}{cc}
1 & 0 \\
\delta^{-2} \rho e^{i h t} & 1
\end{array}\right], \quad V_{4}^{(4)}=\left[\begin{array}{cc}
1 & \delta^{2} \rho e^{-i h t} \\
0 & 1
\end{array}\right], \\
& V_{5}^{(4)}=\left[\begin{array}{cc}
0 & \delta^{2} P_{-}^{-1} e^{-i \Omega_{L} t} \\
-\delta^{-2} P_{-} e^{i \Omega_{L} t} & 0
\end{array}\right], \\
& V_{6}^{(4)}=\left[\begin{array}{cc}
0 & \delta^{2} P_{-} e^{-i \Omega_{L} t} \\
-\delta^{-2} P_{-}^{-1} e^{i \Omega_{L} t} & 0
\end{array}\right], \\
& V_{7}^{(4)}=\left[\begin{array}{cc}
0 & \delta^{2} \\
-\delta^{-2} & 0
\end{array}\right] \text {. }
\end{aligned}
$$

Now the jump matrices decay uniformly in time except on the $\lambda_{L}$ and $\lambda_{R}$ branch cuts and near the point $z_{0}$.

\subsection{When the Residual Region Deformation of $\Sigma^{(0)}$ Fails}

The sign structure of $\Im(h)$ is given by Figure 3.3(a) for sufficiently small $\xi$. There are three points on the real axis from which four branches of $\Im(h)=0$ emanate. The function $h$ behaves quadratically near these points since it is analytic on the real axis away from the branch cuts. Therefore, solving $h^{\prime}(z)=0$ with $\xi$ fixed gives three real-valued solutions for $z$. The middle point is chosen to be $z_{0}$, and the sign structure required by $P^{(4)}$ is satisfied. However, as $\xi$ increases, the leftmost point and $z_{0}$ approach each other, eventually merging when $\xi=\xi_{1}$; see Figure 3.3(b) for a numerically computed example. Here $h$ exhibits cubic behavior, and $\xi_{1}$ and $z_{0}$ may be found by solving the system of real equations

$$
h^{\prime}\left(z_{0}\right)=0, \quad h^{\prime \prime}\left(z_{0}\right)=0 .
$$

For $\xi>\xi_{1}$, the factorization required for $P^{(4)}$ breaks down. Four of the zerolevel curves of $\Im(h)=0$ interchange connections and the point $z_{0}$ ceases to exist (see Figure 3.3(c)). In the upper half-plane there is no way to draw a contour connecting the real axis on the left of the $\lambda_{L}$ cut with the real axis on the right of the $\lambda_{L}$ cut while staying in the region where $\Im(h)<0$. Therefore the matrix $V_{1}^{(4)}$ 


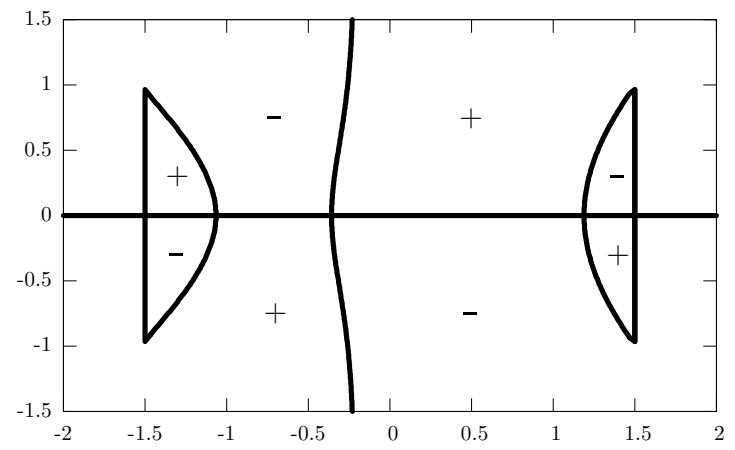

(a) $\xi=0.5$ (residual region)

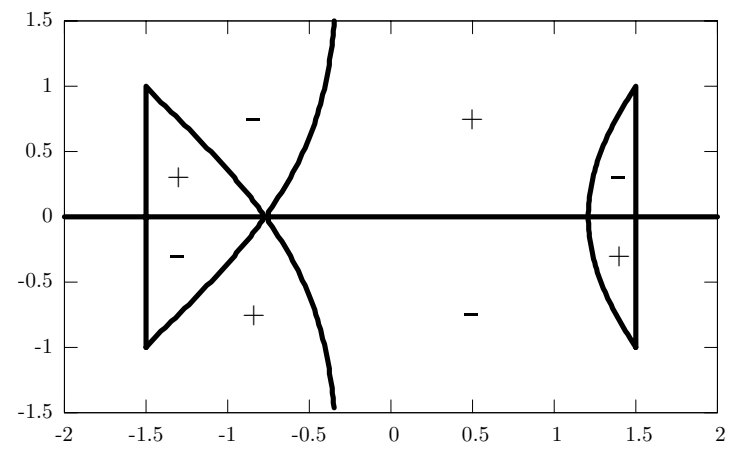

(b) $\xi=\xi_{1} \approx 0.63$ (borderline between residual and transition regions)

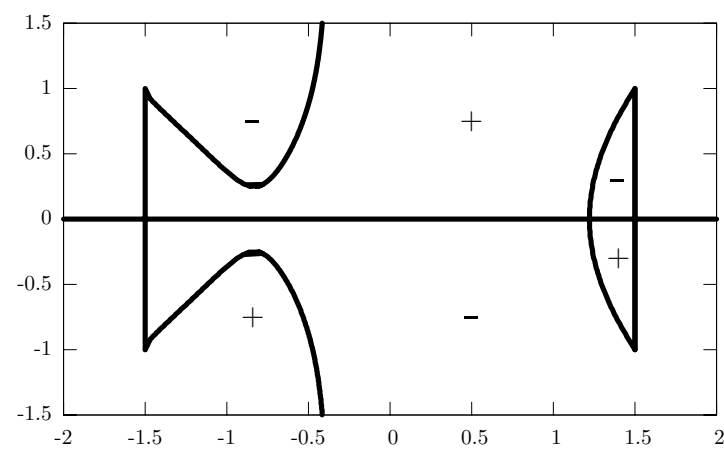

(c) $\xi=0.85$ (transition region)

FIGURE 3.3. Sign structure of $\Im(h)$ for $A=1, \mu=3$.

will not decay along part of its contour. Similarly, in the lower half-plane there is no sea of pluses through which to pass the contour with jump $V_{2}^{(4)}$. This means that there is a range of $\xi$ for which neither the plane-wave region nor residual region 
factorizations will work. This range is referred to as the transition region and will be covered in Section 4.

\section{7 $P^{(4)} \rightarrow P^{(5)}$ : Creating a Constant Jump on the Branch Cuts}

To formulate a solvable model problem, it is necessary to have constant jump matrices on the branch cuts. This is accomplished by the use of another $g$-function. Let

$$
M^{(5)}=M^{(4)} e^{i g \sigma_{3}} .
$$

Here the scalar function $g(z)$ is analytic everywhere off the $\lambda_{L}$ and $\lambda_{R}$ branch cuts. The jump matrices for $M^{(5)}$ are

$$
V_{i}^{(5)}=e^{-i g_{-} \sigma_{3}} V_{i}^{(4)} e^{i g_{+} \sigma_{3}}, \quad i=1, \ldots, 7 .
$$

Explicitly, on the branch cuts these jumps are

$$
\begin{aligned}
V_{5}^{(5)} & =\left[\begin{array}{cc}
0 & \delta^{2} P_{-}^{-1} e^{-i\left(\Omega_{L} t+g_{+}+g_{-}\right)} \\
-\delta^{-2} P_{-} e^{i\left(\Omega_{L} t+g_{+}+g_{-}\right)} & 0
\end{array}\right], \\
V_{6}^{(5)} & =\left[\begin{array}{cc}
0 & \delta^{2} P_{-} e^{-i\left(\Omega_{L} t+g_{+}+g_{-}\right)} \\
-\delta^{-2} P_{-}^{-1} e^{i\left(\Omega_{L} t+g_{+}+g_{-}\right)} & 0
\end{array}\right], \\
V_{7}^{(5)} & =\left[\begin{array}{cc}
0 & \delta^{2} e^{-i\left(g_{+}+g_{-}\right)} \\
-\delta^{-2} e^{i\left(g_{+}+g_{-}\right)} & 0
\end{array}\right] .
\end{aligned}
$$

These jump matrices will be constant if $g$ satisfies the RHP

- $g$ is analytic off $\Sigma^{\omega}=\left(\lambda_{L}\right.$ cut $) \cup\left(\lambda_{R}\right.$ cut $)$,

- $g_{+}+g_{-}= \begin{cases}\omega_{L}-i \ln \left(\delta^{2} / P_{-}\right) & \text {on the } \lambda_{L} \text { cut in the UHP, } \\ \omega_{L}-i \ln \left(\delta^{2} P_{-}\right) & \text {on the } \lambda_{L} \text { cut in the LHP, } \\ -i \ln \left(\delta^{2}\right) & \text { on the } \lambda_{R} \text { cut, }\end{cases}$

- $g \sim g(\infty)$ as $z \rightarrow \infty$,

where $g(\infty)$ is a constant in $z$ and $\omega_{L}$ is a real constant. Let

$$
R(z)=\lambda_{L} \lambda_{R}
$$

Then

$$
\left(\frac{g}{R}\right)_{+}-\left(\frac{g}{R}\right)_{-}= \begin{cases}-\left(\omega_{L}-i \ln \left(\delta^{2} / P_{-}\right)\right) / R_{-} & \text {on } \lambda_{L} \text { cut in UHP } \\ -\left(\omega_{L}-i \ln \left(\delta^{2} P_{-}\right)\right) / R_{-} & \text {on } \lambda_{L} \text { cut in LHP } \\ i \ln \left(\delta^{2}\right) / R_{-} & \text {on } \lambda_{R} \text { cut. }\end{cases}
$$




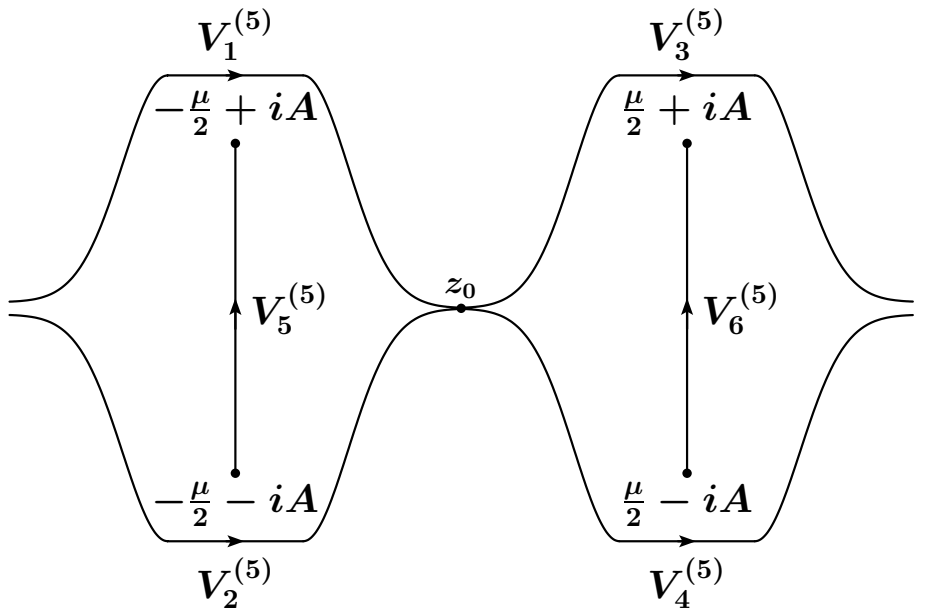

FIGURE 3.4. The RHP $P^{(5)}$ for the residual region.

By the Plemelj formula,

$$
\begin{aligned}
g(z)=-\frac{R(z)}{2 \pi i}[ & \int_{-\mu / 2-i A}^{-\mu / 2} \frac{\omega_{L}-i \ln \left(\delta^{2}(\zeta) P_{-}(\zeta)\right)}{(\zeta-z) R_{-}(\zeta)} \mathrm{d} \zeta \\
& +\int_{-\frac{\mu}{2}}^{-\frac{\mu}{2}+i A} \frac{\omega_{L}-i \ln \left(\delta^{2}(\zeta) / P_{-}(\zeta)\right)}{(\zeta-z) R_{-}(\zeta)} \mathrm{d} \zeta \\
& \left.+\int_{\frac{\mu}{2}-i A}^{\frac{\mu}{2}+i A} \frac{-i \ln \left(\delta^{2}(\zeta)\right)}{(\zeta-z) R_{-}(\zeta)} \mathrm{d} \zeta\right]
\end{aligned}
$$

The integration is done along the branch cuts, and $\omega_{L}$ is chosen so that

$$
g(z)=O(1) \quad \text { as } z \rightarrow \infty .
$$

The limit of $g(z)$ as $z \rightarrow \infty$ is called $g(\infty) . M^{(5)}$ satisfies the RHP

$$
P^{(5)}:\left\{\Sigma^{(5)}=\Sigma^{(4)}, V^{(5)}, e^{i(G(\infty) t+g(\infty)) \sigma_{3}} \text { as } z \rightarrow \infty\right\} .
$$

With this choice of $g, V^{(5)}$ is given by Figure 3.4 and

$$
\begin{array}{rlrl}
V_{1}^{(5)} & =\left[\begin{array}{cc}
1 & \delta^{2} P^{-1} e^{-i(h t+2 g)} \\
0 & 1
\end{array}\right], & V_{2}^{(5)} & =\left[\begin{array}{cc}
1 & 0 \\
\delta^{-2} P^{-1} e^{i(h t+2 g)} & 1
\end{array}\right], \\
V_{3}^{(5)} & =\left[\begin{array}{cc}
1 & 0 \\
\delta^{-2} \rho e^{i(h t+2 g)} & 1
\end{array}\right], & V_{4}^{(5)}=\left[\begin{array}{cc}
1 & \delta^{2} \rho e^{-i(h t+2 g)} \\
0 & 1
\end{array}\right], \\
V_{5}^{(5)}=\left[\begin{array}{cc}
0 & e^{-i\left(\Omega_{L} t+\omega_{L}\right)} \\
-e^{i\left(\Omega_{L} t+\omega_{L}\right)} & 0
\end{array}\right], & V_{6}^{(5)}=\left[\begin{array}{rr}
0 & 1 \\
-1 & 0
\end{array}\right] .
\end{array}
$$



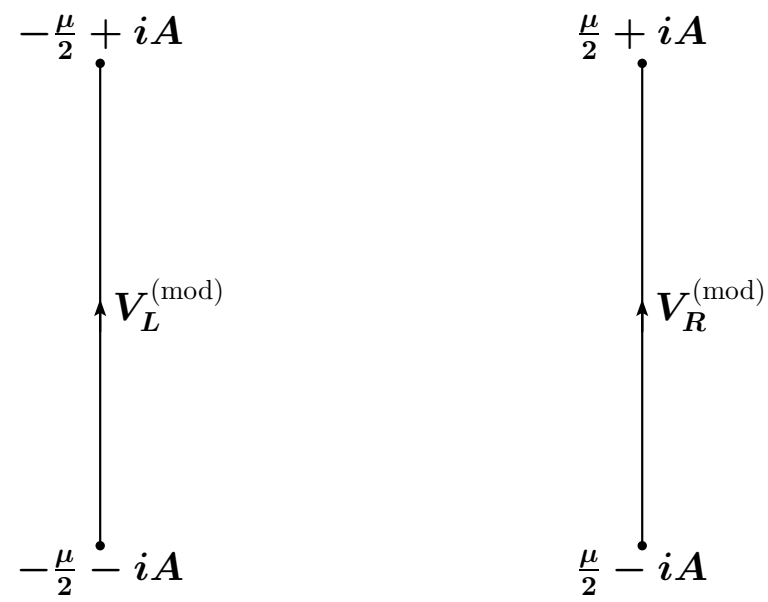

FIGURE 3.5. The RHP $P^{(\bmod )}$ for the residual region.

\subsection{The Model Problem $\boldsymbol{P}^{(\mathrm{mod})}$}

The procedure for calculating $q(x, t)$ continues as in the initial region by defining the matrix $M^{(\mathrm{mod})}$. The main difference is that in the residual region the jump across both the $\lambda_{L}$ and $\lambda_{R}$ branch cuts contribute to the leading-order solution.

$M^{(\mathrm{mod})}$ is defined as the solution to the RHP

$P^{(\text {mod })}:\left\{\Sigma^{(\text {mod })}=\left(\lambda_{L}\right.\right.$ cut $) \cup\left(\lambda_{R}\right.$ cut $), V^{(\text {mod })}, e^{i(G(\infty) t+g(\infty)) \sigma_{3}}$ as $\left.z \rightarrow \infty\right\}$.

See Figure 3.5. The jump matrices are given by

$$
V_{L}^{(\mathrm{mod})}=\left[\begin{array}{cc}
0 & e^{-i\left(\Omega_{L} t+\omega_{L}\right)} \\
-e^{i\left(\Omega_{L} t+\omega_{L}\right)} & 0
\end{array}\right], \quad V_{R}^{(\mathrm{mod})}=\left[\begin{array}{rr}
0 & 1 \\
-1 & 0
\end{array}\right] .
$$

Write $M^{(5)}=M^{(\mathrm{err})} M^{(\mathrm{mod})}$ for large $z$. From equation (A.40),

$$
q(x, t)=-2\left(M_{1}^{(\mathrm{mod})}+M_{1}^{(\mathrm{err})}\right)_{12} e^{i\left[\left(G(\infty)-\mu|\xi|-\mu^{2} / 2+A^{2}\right) t+g(\infty)\right]} .
$$

In Sections 3.9 and 3.10 we show

LEMMA $3.1\left|M_{1}^{(\mathrm{err})}\right|=O\left(t^{-1 / 2}\right)$.

We now solve the model problem using a theta function as in Tovbis, Venakides, and Zhou [15]. Consider the cycles $A_{1}$ and $B_{1}$ defined in Figure 3.6. $A_{1}$ and $B_{1}$ form the canonical homology basis for the torus of genus 1 . To define $\theta(z)$ it is necessary to find a basis for the space of holomorphic differentials on the torus. Each differential has the form

$$
\omega=\frac{c}{\lambda_{L} \lambda_{R}} \mathrm{~d} z
$$

where $c$ is a real constant. Thus the dimension of this space is 1 (in general, it is equal to the genus of the Riemann surface). For the basis element, pick the 


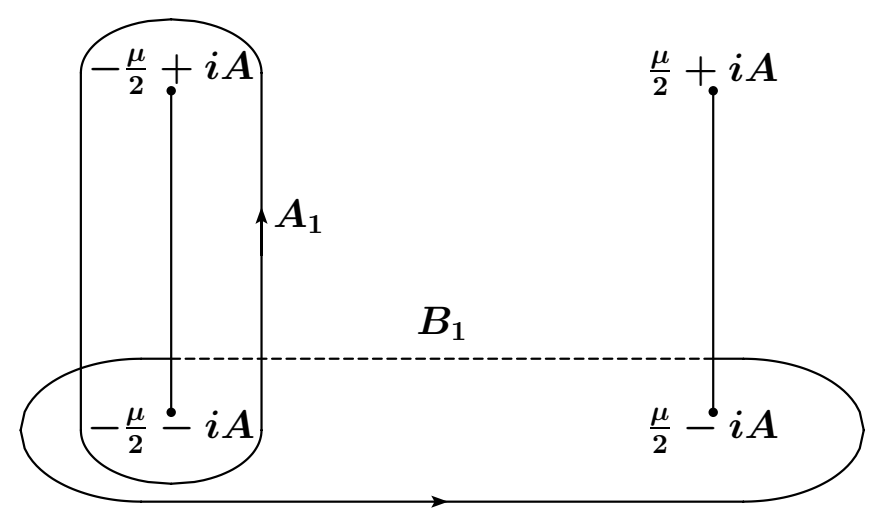

FIGURE 3.6. The canonical homology cycles $\left\{A_{1}, B_{1}\right\}$. The solid lines are on the first sheet, while the dashed line is on the second sheet.

differential $\omega_{1}$ such that

$$
\int_{A_{1}} \omega_{1}=1 .
$$

Also define the Riemann period matrix $\tau$ (in this case the matrix is $1 \times 1$ ) by

$$
\tau=\int_{B_{1}} \omega_{1} .
$$

By Farkas and Kra [11], $\tau$ is purely imaginary and $-i \tau>0$. Define

$$
\theta(z)=\sum_{\ell \in \mathbb{Z}} e^{2 \pi i \ell z+\pi i \ell^{2} \tau} .
$$

$\theta(z)$ has the following properties:

$$
\begin{aligned}
\theta(z) & =\theta(-z), \\
\theta(z+1) & =\theta(z), \\
\theta(z+\tau) & =e^{-2 \pi i z-\pi i \tau} \theta(z) .
\end{aligned}
$$

Denote

$$
u(z)=\int_{\mu / 2-i A}^{z} \omega_{1} .
$$

Next, define

$$
\begin{aligned}
\mathcal{M}(z, d) & =\left(\mathcal{M}_{1}, \mathcal{M}_{2}\right) \\
& =\left(\frac{\theta\left(\frac{\Omega_{L}}{2 \pi} t+\frac{\omega}{2 \pi}+u(z)+d\right)}{\theta(u(z)+d)}, \frac{\theta\left(\frac{\Omega_{L}}{2 \pi} t+\frac{\omega}{2 \pi}-u(z)+d\right)}{\theta(-u(z)+d)}\right),
\end{aligned}
$$


where $d \in \mathbb{C}$ is to be determined. $\mathcal{M}(z, d)$ is well-defined even though $u(z)$ is multivalued. However, $\mathcal{M}(z, d)$ has singularities that depend on the choice of $d$. If $\tilde{z}$ is a zero of $\theta(u(z))$,

$$
\frac{\mathrm{d} u}{\mathrm{~d} z}=\frac{q}{\lambda_{L} \lambda_{R}}=O\left((z-\tilde{z})^{-1 / 2}\right)
$$

implies

$$
u(z)-u(\tilde{z})=O\left((z-\tilde{z})^{1 / 2}\right)
$$

near $\tilde{z}$. Furthermore, the zero of $\theta(u)=O(u(z)-u(\tilde{z}))$ is simple by the argument principle, so the singularities of $\mathcal{M}$ are of order $\frac{1}{2}$. The identity

$$
u_{+}(z)+u_{-}(z)= \begin{cases}-\tau-n, & z \in \lambda_{L} \text { cut, } n \in \mathbb{Z}, \\ 0, & z \in \lambda_{R} \text { cut },\end{cases}
$$

can be used to compute the jump of $\mathcal{M}$. On the $\lambda_{L}$ cut:

$$
\begin{aligned}
\mathcal{M}_{+}= & \left(\frac{\theta\left(\frac{\Omega_{L}}{2 \pi} t+\frac{\omega}{2 \pi}-u_{-}-n-\tau+d\right)}{\theta\left(-u_{-}-n-\tau+d\right)}, \frac{\theta\left(\frac{\Omega_{L}}{2 \pi} t+\frac{\omega}{2 \pi}+u_{-}+n+\tau+d\right)}{\theta\left(u_{-}+n+\tau+d\right)}\right) \\
= & \left(\frac{\theta\left(\frac{\Omega_{L}}{2 \pi} t+\frac{\omega}{2 \pi}-u_{-}+d\right) e^{-2 \pi i\left(-\Omega_{L} t / 2 \pi-\omega_{L} / 2 \pi+u_{-}-d\right)-\pi i \tau}}{\theta\left(-u_{-}+d\right) e^{-2 \pi i\left(u_{-}-d\right)-\pi i \tau}},\right. \\
& \left.\frac{\theta\left(\frac{\Omega_{L}}{2 \pi} t+\frac{\omega}{2 \pi}+u_{-}+d\right) e^{-2 \pi i\left(\Omega_{L} t / 2 \pi+\omega_{L} / 2 \pi+u_{-}+d\right)-\pi i \tau}}{\theta\left(u_{-}+d\right) e^{-2 \pi i\left(u_{-}+d\right)-\pi i \tau}}\right) \\
= & \left(\mathcal{M}_{2-} e^{i\left(\Omega_{L} t+\omega_{L}\right)}, \mathcal{M}_{1-} e^{-i\left(\Omega_{L} t+\omega_{L}\right)}\right) \\
= & \mathcal{M}_{-}\left[\begin{array}{cc}
0 & e^{-i\left(\Omega_{L} t+\omega_{L}\right)} \\
e^{i\left(\Omega_{L} t+\omega_{L}\right)} & 0
\end{array}\right] .
\end{aligned}
$$

On the $\lambda_{R}$ cut

$$
\begin{aligned}
\mathcal{M}_{+} & =\left(\frac{\theta\left(\frac{\Omega_{L}}{2 \pi} t+\frac{\omega}{2 \pi}-u_{-}+d\right)}{\theta\left(-u_{-}+d\right)}, \frac{\theta\left(\frac{\Omega_{L}}{2 \pi} t+\frac{\omega}{2 \pi}+u_{-}+d\right)}{\theta\left(u_{-}+d\right)}\right) \\
& =\left(\mathcal{M}_{2-}, \mathcal{M}_{1-}\right) \\
& =\mathcal{M}_{-}\left[\begin{array}{ll}
0 & 1 \\
1 & 0
\end{array}\right] .
\end{aligned}
$$

Thus $\mathcal{M}$ comes close to satisfying the model RHP, but there is a minus sign lacking in the 21-entry of the jump matrix. Now set

$$
\gamma(z)=\left(\frac{\left(z-\left(\frac{\mu}{2}+i A\right)\right) \cdot\left(z-\left(-\frac{\mu}{2}+i A\right)\right)}{\left(z-\left(\frac{\mu}{2}-i A\right)\right) \cdot\left(z-\left(-\frac{\mu}{2}-i A\right)\right)}\right)^{1 / 4},
$$


chosen so that $\gamma(z) \sim 1$ as $z \rightarrow \infty$ and $\gamma(z)$ has branch cuts along the $\lambda_{L}$ and $\lambda_{R}$ cuts. $\gamma_{+}=i \gamma_{-}$along these cuts. Define

$$
\mathcal{N}(z, d)=\frac{1}{2}\left[\begin{array}{cc}
\left(\gamma+\gamma^{-1}\right) \mathcal{M}_{1}(z, d) & -i\left(\gamma-\gamma^{-1}\right) \mathcal{M}_{2}(z, d) \\
i\left(\gamma-\gamma^{-1}\right) \mathcal{M}_{1}(z,-d) & \left(\gamma+\gamma^{-1}\right) \mathcal{M}_{2}(z,-d)
\end{array}\right] .
$$

Now $\mathcal{N}$ satisfies the desired jump condition on the $\lambda_{L}$ cut:

$$
\begin{aligned}
\mathcal{N}_{+} & =\frac{1}{2}\left[\begin{array}{cc}
\left(\gamma_{+}+\gamma_{+}^{-1}\right) \mathcal{M}_{1+}(z, d) & -i\left(\gamma_{+}-\gamma_{+}^{-1}\right) \mathcal{M}_{2+}(z, d) \\
i\left(\gamma_{+}-\gamma_{+}^{-1}\right) \mathcal{M}_{1+}(z,-d) & \left(\gamma_{+}+\gamma_{+}^{-1}\right) \mathcal{M}_{2+}(z,-d)
\end{array}\right] \\
(3.56) & =\frac{1}{2}\left[\begin{array}{cc}
i\left(\gamma_{-}-\gamma_{-}^{-1}\right) \mathcal{M}_{2-}(z, d) e^{i\left(\Omega_{L} t+\omega_{L}\right)} & \left(\gamma_{-}+\gamma_{-}^{-1}\right) \mathcal{M}_{1-}(z, d) e^{-i\left(\Omega_{L} t+\omega_{L}\right)} \\
-\left(\gamma_{-}+\gamma_{-}^{-1}\right) \mathcal{M}_{2-}(z,-d) e^{i\left(\Omega_{L} t+\omega_{L}\right)} & i\left(\gamma_{-}-\gamma_{-}^{-1}\right) \mathcal{M}_{1-}(z,-d) e^{-i\left(\Omega_{L} t+\omega_{L}\right)}
\end{array}\right] \\
& =\mathcal{N}_{-}\left[\begin{array}{cc}
0 & e^{-i\left(\Omega_{L} t+\omega_{L}\right)} \\
-e^{i\left(\Omega_{L} t+\omega_{L}\right)} & 0
\end{array}\right] .
\end{aligned}
$$

Similarly, on the $\lambda_{R}$ cut,

$$
\mathcal{N}_{+}=\mathcal{N}_{-}\left[\begin{array}{rr}
0 & 1 \\
-1 & 0
\end{array}\right]
$$

Now $d$ is chosen so the poles of $\mathcal{N}$ from $\mathcal{M}$ coincide with the zeros of $\mathcal{N}$ from $\gamma-\gamma^{-1}$. Let $z_{1}$ be the unique zero of $\gamma-\gamma^{-1}$; also let $X_{1}\left(z_{1}\right)$ and $X_{2}\left(z_{1}\right)$ be the preimages of $z_{1}$ on the first and second sheet of the elliptic surface, respectively. Choose

$$
d=-\int_{-\mu / 2-i A}^{X_{2}\left(z_{1}\right)} \omega_{1} .
$$

By Farkas and Kra [11], $X_{1}\left(z_{1}\right)$ is the zero of $\theta(-u(z)+d)$, and $X_{2}\left(z_{1}\right)$ is the zero of $\theta(u(z)+d)$. Since $\mathcal{N}$ is defined on the first sheet, the only singularities are in the 12- and 21-positions, which are canceled by the zero from $\gamma-\gamma^{-1} \mathcal{N}$ is also analytic off the $\lambda_{L}$ and $\lambda_{R}$ cuts. Thus the solution to the RHP $P^{(\bmod )}$ is

$$
M^{(\bmod )}(z)=e^{i(G(\infty) t+g(\infty)) \sigma_{3}} \mathcal{N}^{-1}(\infty) \mathcal{N}(z) .
$$

Note that

$$
\gamma(z)-\gamma^{-1}(z)=-2 i A z^{-1}+O\left(z^{-2}\right) \quad \text { as } z \rightarrow \infty
$$

and

$$
\mathcal{N}^{-1}(\infty)=\left[\begin{array}{cc}
\frac{1}{\mathcal{M}_{1}(\infty, d)} & 0 \\
0 & \frac{1}{\mathcal{M}_{2}(\infty,-d)}
\end{array}\right]
$$

Therefore

$$
\left(M_{1}^{(\mathrm{mod})}\right)_{12}=-A \frac{\mathcal{M}_{2}(\infty, d)}{\mathcal{M}_{1}(\infty, d)} e^{i[G(\infty) t+g(\infty)]}
$$



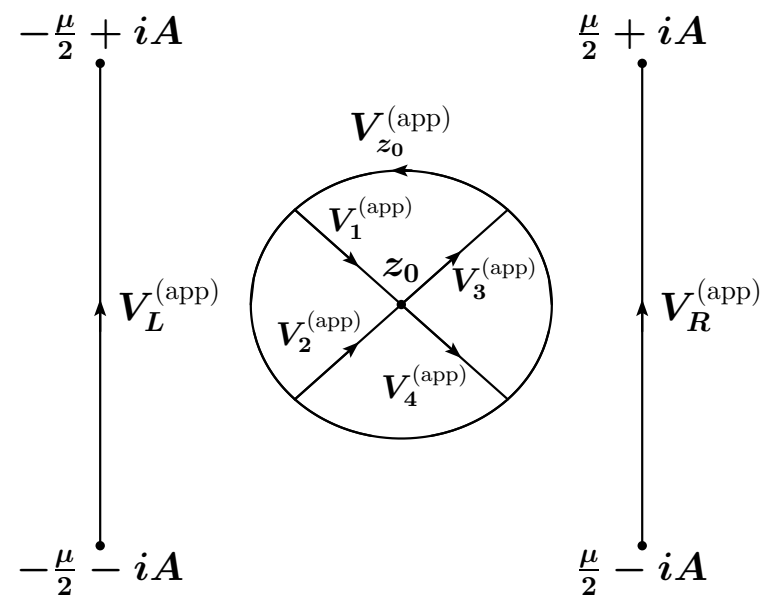

FIGURE 3.7. The RHP $P^{(\text {app })}$ for the residual region with enlarged detail around $z_{0}$.

\subsection{The Error Problem $\boldsymbol{P}^{(\mathrm{err})}$}

As in the plane-wave region, the proof of the error estimate requires taking into account the nonuniform decay near $z_{0}$. Therefore, define

$$
M^{(5)}=M^{(\mathrm{err})} M^{(\mathrm{app})} \text {. }
$$

$M^{(\text {app) }}$ has the same jumps as $M^{(\bmod )}$ plus an additional jump near $z_{0}$. Pick $\varepsilon$ sufficiently small so that $r_{z_{0}}^{\varepsilon}=\left\{z:\left|z-z_{0}\right|=\varepsilon\right\}$ does not intersect $\Sigma^{(\text {mod) }}$. Define $M^{(\text {app })}$ by

$$
M^{(\text {app })}= \begin{cases}\text { parametrix of } V^{(5)}, & \left|z-z_{0}\right| \leq \varepsilon, \\ M^{(\text {mod })}, & \varepsilon \leq\left|z-z_{0}\right| .\end{cases}
$$

This matrix function satisfies the RHP

$$
P^{(\mathrm{app})}:\left\{\Sigma^{(\mathrm{app})}\left(\text { see Figure 3.7), } V^{(\mathrm{app})}, e^{i(G(\infty) t+g(\infty)) \sigma_{3}} \text { as } z \rightarrow \infty\right\},\right.
$$

with $V^{(\text {app })}$ given by

$$
\begin{array}{cc}
V_{L}^{(\text {app })}=V_{L}^{(\mathrm{mod})}, & V_{R}^{(\mathrm{app})}=V_{R}^{(\mathrm{mod})}, \\
V_{i}^{(\mathrm{app})}=V_{i}^{(5)}, & i=1,2,3,4 .
\end{array}
$$

There is also an additional jump $V_{z_{0}}^{(\text {app })}$ on $r_{z_{0}}^{\varepsilon}$ having the form $I+O\left(t^{-1 / 2}\right)$ as shown below. See Figure 3.7.

The definition of $M^{(\mathrm{app})}$ and equation (3.63) fix $M^{(\mathrm{err})}$, which satisfies the RHP

$$
P^{(\mathrm{err})}:\left\{\Sigma^{(\mathrm{err})} \text { (see Figure 3.8), } V^{(\mathrm{err})}, I \text { as } z \rightarrow \infty\right\},
$$




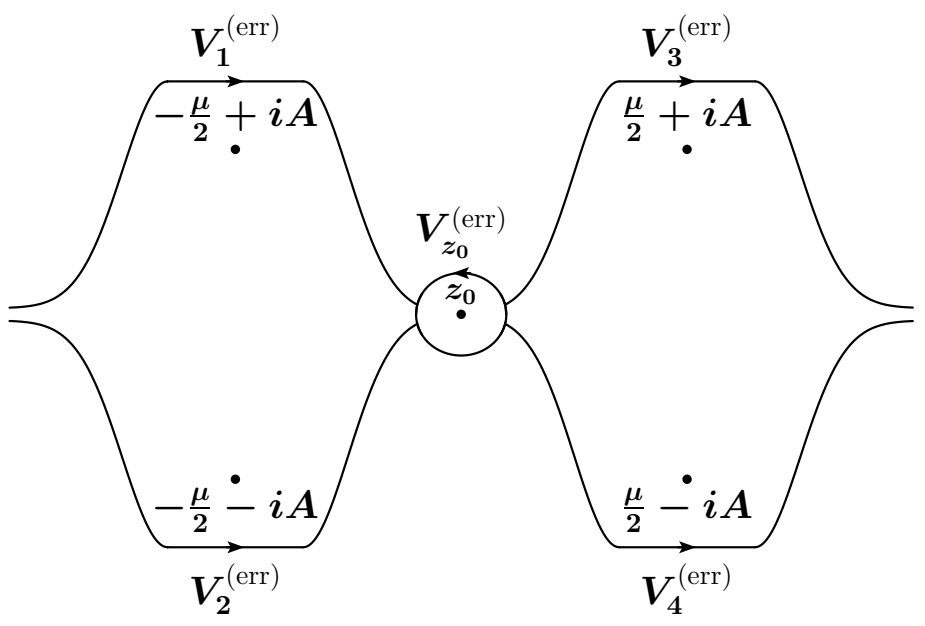

FIGURE 3.8. The RHP $P^{(\text {err })}$ for the residual region.

with $V^{\text {(err) }}$ defined by Figure 3.8 and

$$
\begin{array}{ll}
V_{i}^{(\mathrm{err})}=M^{(\mathrm{app})} V_{i}^{(5)}\left(M^{(\mathrm{app})}\right)^{-1} & \text { outside } r_{z_{0}}^{\varepsilon}, i=1,2,3,4, \\
V_{z_{0}}^{(\mathrm{err})}=M_{-}^{(\mathrm{app})}\left(V_{z_{0}}^{(\mathrm{app})}\right)^{-1}\left(M_{-}^{(\mathrm{app})}\right)^{-1} & \text { on } r_{z_{0}}^{\varepsilon} .
\end{array}
$$

\subsection{The Error Estimate}

The proof of Lemma 3.1 follows the proof of Lemma 2.1 in the plane-wave region. The parametrix near $z_{0}$ inside the circle $r_{z_{0}}^{\varepsilon}$ has the same jump conditions as in the plane-wave region except that $f(z)$ is replaced by $h(z)=f(z)+2 G(z) \cdot g(z)$ has a different form but is still analytic near $z_{0}$ and does not change the process. The construction of the parametrix proceeds as in Appendix B with $f(z)$ replaced with $h(z)$, yielding

$$
V_{z_{0}}^{(\mathrm{err})}=I+O\left(t^{-1 / 2}\right)
$$

as $t \rightarrow \infty$. Continuing as in Section 2.9 yields

$$
M_{1}^{(\mathrm{err})}=O\left(t^{-1 / 2}\right)
$$

which is Lemma 3.1. This, along with equations (3.39) and (3.62) proves Theorem 1.2.

\section{Solution in the Transition Region}

The leading-order solution for $q(x, t)$ has now been computed for the planewave and residual regions. As seen in Section 3.6, there is a range of $|\xi|$ between these two regions where neither method works. These $|\xi|$-values constitute a transition region in which it is necessary to use a third factorization. The resulting 
RHP lies on three main bands, and the leading-order solution is found to have two nonlinear oscillations.

\subsection{Overview of the Solution in the Transition Region}

We start with the RHP $P^{(3)}$ from the residual region. As $|\xi|$ increases to the boundary of the residual region, the point $z_{0}$ where $\Im(h)$ changes sign immediately above the real axis is lost, as shown in Figure 3.3(c). Now the jump $V_{1}^{(3)}$ grows exponentially in time on the contour near $z_{0}$ instead of decaying to the identity. A new set of transformations is introduced to deal with this growth by keeping part of the contour near $z_{0}$ in the model problem.

- $P^{(3)} \rightarrow P^{(4)}$ : The RHP $P^{(4)}$ is defined in Section 4.2 using a new factorization of the jump on the contour near $z_{0}$.

- $P^{(4)} \rightarrow P^{(5)}$ : The exponential growth of the jump near $z_{0}$ is removed by the function $G(z)$ in Section 4.3, leading to the RHP $P^{(5)}$.

- $P^{(5)} \rightarrow P^{(6)}$ : Using the function $g(z), P^{(6)}$ is defined in Section 4.4 so that the jumps on the three branch cuts are constant in $z$.

The three-banded model problem $P^{(\mathrm{mod})}$ is defined and solved explicitly in terms of theta functions in Section 4.5. The approximate and error problems $P^{(\mathrm{app})}$ and $P^{\text {(err) }}$ are defined in Section 4.6. The approximate RHP consists of jumps on the three bands of the model RHP plus jumps near $z_{0}$ and the endpoints $\alpha$ and $\bar{\alpha}$ of the center band. The error analysis near $\alpha$ and $\bar{\alpha}$ require the construction of parametrices, which is done in Section 4.7. The parametrix near $z_{0}$ is computed in Section 4.8, and the error estimate is outlined in Section 4.9.

\section{2 $P^{(3)} \rightarrow P^{(4)}$ : New Factorization of $V^{(3)}$}

Start with the RHP $P^{(3)}$ from the residual region as defined in Section 3.4. The point $z_{0} \in(-\mu / 2, \mu / 2)$ is unknown at this stage and will be determined after the introduction of a $g$-function. Recall that when the $g$-function from the residual region is used in the transition region, the norms of two of the jump matrices around $z_{0}$ grow exponentially. A new factorization of $V_{1}^{(3)}$ and $V_{2}^{(3)}$ near $z_{0}$ and a different $g$-function will remove this difficulty. First, define

$$
\begin{aligned}
& V_{i}^{(4)}=V_{i}^{(3)}, \quad i=1,2,3,4, \\
& V_{5}^{(4)}=\left[\begin{array}{cc}
1 & 0 \\
\delta^{-2} P e^{i f t} & 1
\end{array}\right], \quad V_{6}^{(4)}=\left[\begin{array}{cc}
1 & \delta^{2} P e^{-i f t} \\
0 & 1
\end{array}\right], \\
& V_{7}^{(4)}=\left[\begin{array}{cc}
0 & \delta^{2} P^{-1} e^{-i f t} \\
-\delta^{-2} P e^{i f t} & 0
\end{array}\right], \quad V_{8}^{(4)}=\left[\begin{array}{cc}
0 & -\delta^{2} P e^{-i f t} \\
\delta^{-2} P^{-1} e^{i f t} & 0
\end{array}\right], \\
& V_{9}^{(4)}=V_{5}^{(3)}, \quad V_{10}^{(4)}=V_{6}^{(3)}, \quad V_{11}^{(4)}=V_{7}^{(3)} .
\end{aligned}
$$

Now deform the contour in the upper half-plane with jump $V_{1}^{(3)}$ so that it passes through the point $\alpha$, which will be found in Section 4.3. In a similar manner, 


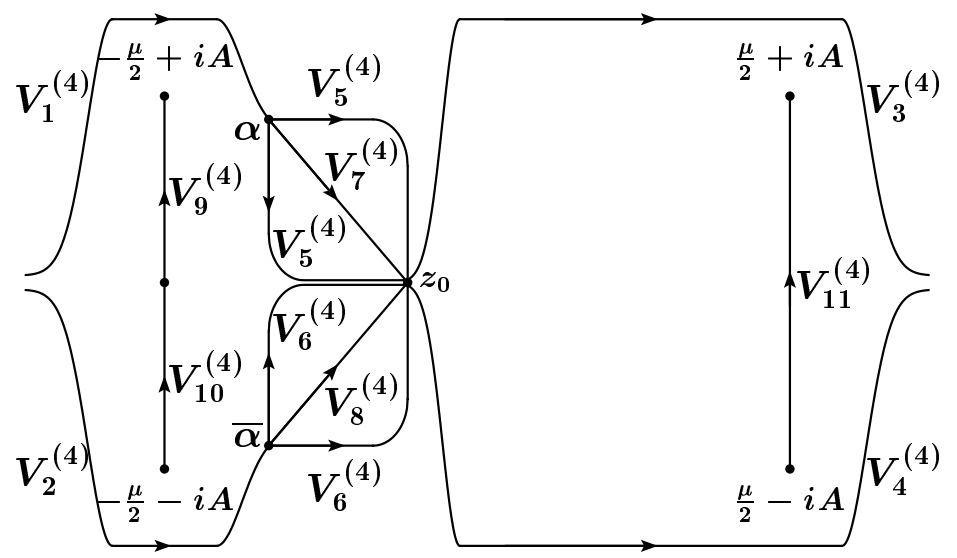

FIGURE 4.1. The RHP $P^{(4)}$ for the transition region.

deform the contour in the lower half-plane with jump $V_{2}^{(3)}$ so that it passes through $\bar{\alpha}$. Along the contour from $\alpha$ to $z_{0}$, use the factorization

$$
V_{1}^{(3)}=V_{5}^{(4)} V_{7}^{(4)} V_{5}^{(4)} \text {. }
$$

Similarly, in the lower half-plane along the contour from $\bar{\alpha}$ to $z_{0}$ use the factorization

$$
V_{2}^{(3)}=V_{6}^{(4)} V_{8}^{(4)} V_{6}^{(4)}
$$

The new RHP is

$$
P^{(4)}:\left\{\Sigma^{(4)} \text { (see Figure 4.1), } V^{(4)}, I \text { as } z \rightarrow \infty\right\} .
$$

\section{3 $P^{(4)} \rightarrow P^{(5)}$ : The $g$-Function Mechanism}

One of the contours from $\alpha$ to $\bar{\alpha}$ will contribute to the leading-order term of $q(x, t)$. With this in mind, it is expedient to define the function $\lambda_{C}(z)$ with a branch cut on this center contour between the left and right branch cuts. Let

$$
\lambda_{C}(z)=((z-\alpha)(z-\bar{\alpha}))^{1 / 2} .
$$

The branch cut is taken to be two straight-line segments from $\alpha$ to $z_{0}$ to $\bar{\alpha}$. The sheet is chosen so that $\lambda_{C} \sim z$ as $z \rightarrow \infty$.

A $g$-function is now used to remove the exponential growth in time along the three branch cuts. Let $G(z)$ be a to-be-determined scalar function analytic off the branch cuts. Then define

$$
M^{(5)}=M^{(4)} e^{i G \sigma_{3} t} .
$$


$M^{(5)}$ is the solution to the RHP

$$
P^{(5)}:\left\{\Sigma^{(5)}=\Sigma^{(4)}, V^{(5)}=e^{-i G_{-} \sigma_{3} t} V^{(4)} e^{i G_{+} \sigma_{3} t}, e^{i G(\infty) \sigma_{3} t} \text { as } z \rightarrow \infty\right\} .
$$

$V^{(5)}$ is given explicitly by

$$
\begin{aligned}
& V_{1}^{(5)}=\left[\begin{array}{cc}
1 & \delta^{2} P^{-1} e^{-i(f+2 G) t} \\
0 & 1
\end{array}\right], \quad V_{2}^{(5)}=\left[\begin{array}{cc}
1 & 0 \\
\delta^{-2} P^{-1} e^{i(f+2 G) t} & 1
\end{array}\right], \\
& V_{3}^{(5)}=\left[\begin{array}{cc}
1 & 0 \\
\delta^{-2} \rho e^{i(f+2 G) t} & 1
\end{array}\right], \quad V_{4}^{(5)}=\left[\begin{array}{cc}
1 & \delta^{2} \rho e^{-i(f+2 G) t} \\
0 & 1
\end{array}\right], \\
& V_{5}^{(5)}=\left[\begin{array}{cc}
1 & 0 \\
\delta^{-2} P e^{i(f+2 G) t} & 1
\end{array}\right], \quad V_{6}^{(5)}=\left[\begin{array}{cc}
1 & \delta^{2} P e^{-i(f+2 G) t} \\
0 & 1
\end{array}\right], \\
& V_{7}^{(5)}=\left[\begin{array}{cc}
0 & \delta^{2} P^{-1} e^{-i\left(f+G_{+}+G_{-}\right) t} \\
-\delta^{-2} P e^{i\left(f+G_{+}+G_{-}\right) t} & 0
\end{array}\right], \\
& V_{8}^{(5)}=\left[\begin{array}{cc}
0 & -\delta^{2} P e^{-i\left(f+G_{+}+G_{-}\right) t} \\
\delta^{-2} P^{-1} e^{i\left(f+G_{+}+G_{-}\right) t} & 0
\end{array}\right], \\
& V_{9}^{(5)}=\left[\begin{array}{cc}
0 & \delta^{2} P_{-}^{-1} e^{-i\left(f+G_{+}+G_{-}\right) t} \\
-\delta^{-2} P_{-} e^{i\left(f+G_{+}+G_{-}\right) t} & 0
\end{array}\right], \\
& V_{10}^{(5)}=\left[\begin{array}{cc}
0 & \delta^{2} P_{-} e^{-i\left(f+G_{+}+G_{-}\right) t} \\
-\delta^{-2} P_{-}^{-1} e^{i\left(f+G_{+}+G_{-}\right) t} & 0
\end{array}\right], \\
& V_{11}^{(5)}=\left[\begin{array}{cc}
0 & \delta^{2} e^{-i\left(G_{+}+G_{-}\right) t} \\
-\delta^{-2} e^{i\left(G_{+}+G_{-}\right) t} & 0
\end{array}\right] .
\end{aligned}
$$

$G(z)$ is defined to eliminate any growth or decay in time in the jump matrices on the three branch cuts. The RHP satisfied by $G$ is

- $G$ is analytic off the $\lambda_{L}, \lambda_{C}$, and $\lambda_{R}$ branch cuts.

- $\begin{cases}f+G_{+}+G_{-}=\Omega_{L} & \text { on the } \lambda_{L} \text { branch cut, } \\ f+G_{+}+G_{-}=\Omega_{C} & \text { on the } \lambda_{C} \text { branch cut, } \\ G_{+}+G_{-}=0 & \text { on the } \lambda_{R} \text { branch cut, }\end{cases}$

- $G \sim G(\infty)$ as $z \rightarrow \infty$,

where $\Omega_{L}, \Omega_{C}$, and $G(\infty)$ are real constants. Define

$$
R(z)=\lambda_{L}(z) \lambda_{C}(z) \lambda_{R}(z) \text {. }
$$

Observe that

$$
R(z)=z^{3}-\Re(\alpha) z^{2}+\left(A^{2}-\frac{\mu^{2}}{4}+\frac{1}{2}[\Im(\alpha)]^{2}\right) z+O(1) \quad \text { as } z \rightarrow \infty .
$$

Using the same reasoning as in the residual region,

$$
G(z)=\frac{R(z)}{2 \pi i}\left[\int_{\lambda_{L} \text { cut }} \frac{f(\zeta)-\Omega_{L}}{R(\zeta)(\zeta-z)} \mathrm{d} \zeta+\int_{\lambda_{C} \text { cut }} \frac{f(\zeta)-\Omega_{C}}{R(\zeta)(\zeta-z)} \mathrm{d} \zeta\right]
$$


It still remains to find $\alpha, z_{0}, \Omega_{L}$, and $\Omega_{C}$. As in the residual region, let

$$
h(z)=f(z)+2 G(z) .
$$

Near $\alpha$,

$$
h(z)=C_{0}+C_{1}(z-\alpha)^{1 / 2}+O\left((z-\alpha)^{3 / 2}\right),
$$

where $C_{0}$ is a real constant and $C_{1}$ is a complex constant. The desired sign structure of $\Im(h)$ requires three branches of $\Im(h)=0$ emanating from $\alpha$. Therefore $C_{1}=0$, or

$$
\left.(z-\alpha)^{1 / 2} h^{\prime}(z)\right|_{z=\alpha}=0 .
$$

Condition (4.12) gives two real equations. The sign structure also requires $\mathfrak{I}(h)$ to be positive along the contours in the upper half-plane with jumps $V_{5}^{(5)}$ (with corresponding conditions in the lower half-plane). This is equivalent to requiring $\Im(h)=0$ immediately above $z_{0}$. Writing $z=z_{0}+i v$ near $z_{0}$, this condition becomes

$$
\left.\Im\left(\frac{1}{v} h\left(z_{0}+i v\right)\right)\right|_{\nu=0}=0 .
$$

Also, $G(z)=O\left(z^{2}\right)$ as $z \rightarrow \infty$, and removing this singularity at infinity gives the two equations

$$
\begin{aligned}
& \int_{\lambda_{L} \text { cut }} \frac{f(\zeta)-\Omega_{L}}{R(\zeta)} \mathrm{d} \zeta+\int_{\lambda_{C} \text { cut }} \frac{f(\zeta)-\Omega_{C}}{R(\zeta)} \mathrm{d} \zeta=0, \\
& \int_{\lambda_{L} \text { cut }} \frac{f(\zeta)-\Omega_{L}}{R(\zeta)} \zeta \mathrm{d} \zeta+\int_{\lambda_{C} \text { cut }} \frac{f(\zeta)-\Omega_{C}}{R(\zeta)} \zeta \mathrm{d} \zeta \\
& \quad-\Re(\alpha)\left(\int_{\lambda_{L} \text { cut }} \frac{f(\zeta)-\Omega_{L}}{R(\zeta)} \mathrm{d} \zeta+\int \frac{f(\zeta)-\Omega_{C}}{R(\zeta)} \mathrm{d} \zeta\right)=0 .
\end{aligned}
$$

Now equations (4.12), (4.13), (4.14), and (4.15) give five real equations for the five real unknowns $\Re(\alpha), \Im(\alpha), z_{0}, \Omega_{L}$, and $\Omega_{C}$. For fixed $A$ and $\mu$, the values of $\xi$ for which these equations are solvable with $-\mu / 2<\Re(\alpha)<\mu / 2$ and $0<\Im(\alpha)<A$ comprise the transition region. Now, using equation (4.8),

$$
\begin{aligned}
G(\infty) & =\frac{-1}{2 \pi i}\left[\int_{\lambda_{L} \text { cut }} \frac{f(\zeta)-\Omega_{L}}{R(\zeta)} \zeta^{2} \mathrm{~d} \zeta+\int_{\lambda_{C} \text { cut }} \frac{f(\zeta)-\Omega_{C}}{R(\zeta)} \zeta^{2} \mathrm{~d} \zeta\right. \\
& -\Re(\alpha)\left(\int_{\lambda_{L} \text { cut }} \frac{f(\zeta)-\Omega_{L}}{R(\zeta)} \zeta \mathrm{d} \zeta+\int_{\lambda_{C} \text { cut }} \frac{f(\zeta)-\Omega_{C}}{R(\zeta)} \zeta \mathrm{d} \zeta\right) \\
& \left.+\left(A^{2}-\frac{\mu^{2}}{4}+\frac{1}{2}[\Im(\alpha)]^{2}\right)\left(\int_{\lambda_{L} \text { cut }} \frac{f(\zeta)-\Omega_{L}}{R(\zeta)} \mathrm{d} \zeta+\int_{\lambda_{C} \text { cut }} \frac{f(\zeta)-\Omega_{C}}{R(\zeta)} \mathrm{d}\right)\right]
\end{aligned}
$$




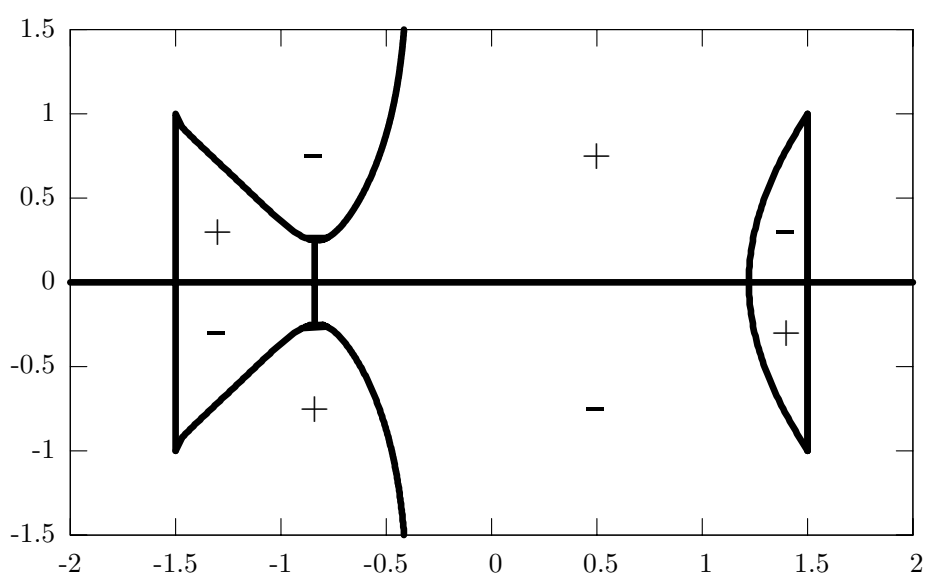

FIGURE 4.2. $\Im(h)$ for $A=1, \mu=3, \xi=0.85$ (in the transition region). $\Im(h)$ is 0 on the contour connecting $\alpha$ and $\bar{\alpha}$ but has a jump across the $\lambda_{L}$ and $\lambda_{R}$ branch cuts.

With this choice of $G$, the jump matrices $V_{i}^{(5)}$ simplify to

$$
\begin{gathered}
V_{1}^{(5)}=\left[\begin{array}{cc}
1 & \delta^{2} P^{-1} e^{-i h t} \\
0 & 1
\end{array}\right], \quad V_{2}^{(5)}=\left[\begin{array}{cc}
1 & 0 \\
\delta^{-2} P^{-1} e^{i h t} & 1
\end{array}\right], \quad V_{3}^{(5)}=\left[\begin{array}{cc}
1 & 0 \\
\delta^{-2} \rho e^{i h t} & 1
\end{array}\right], \\
V_{4}^{(5)}=\left[\begin{array}{ccc}
1 & \delta^{2} \rho e^{-i h t} \\
0 & 1
\end{array}\right], \quad V_{5}^{(5)}=\left[\begin{array}{cc}
1 & 0 \\
\delta^{-2} P e^{i h t} & 1
\end{array}\right], \quad V_{6}^{(5)}=\left[\begin{array}{cc}
1 & \delta^{2} P e^{-i h t} \\
0 & 1
\end{array}\right], \\
V_{7}^{(5)}=\left[\begin{array}{cc}
0 & \delta^{2} P_{-}^{-1} e^{-i \Omega_{L} t} \\
-\delta^{-2} P_{-} e^{i \Omega_{L} t} & 0
\end{array}\right], \\
V_{8}^{(5)}=\left[\begin{array}{cc}
0 & \delta^{2} P_{-} e^{-i \Omega_{L} t} \\
-\delta^{-2} P_{-}^{-1} e^{i \Omega_{L} t} & 0
\end{array}\right], \\
V_{9}^{(5)}=\left[\begin{array}{cc}
0 & \delta^{2} P^{-1} e^{-i \Omega_{C} t} \\
-\delta^{-2} P e^{i \Omega_{C} t} & 0
\end{array}\right], \\
\left.0 \quad \begin{array}{cc}
0 \\
\delta_{10}^{2} P e^{-i \Omega_{C} t}
\end{array}\right], \quad V_{11}^{(5)}=\left[\begin{array}{cc}
0 & \delta^{2} \\
-\delta^{-2} & 0
\end{array}\right] .
\end{gathered}
$$

Finally, $\Sigma^{(5)}$ is chosen so that the contours with jumps $V_{1}^{(5)}, V_{4}^{(5)}$, and $V_{6}^{(5)}$ pass through regions where $\Im h<0$, and the contours with jumps $V_{2}^{(5)}, V_{3}^{(5)}$, and $V_{5}^{(5)}$ pass through regions where $\Im h>0$. See Figure 4.2 for an example of the numerically calculated sign structure of $\Im(h)$.

\section{4 $P^{(5)} \rightarrow P^{(6)}$ : Reduction of the Jumps on Branch Cuts to Constants}

The jump matrices on the branch cuts are reduced to constants by writing

$$
M^{(6)}(z)=M^{(5)}(z) e^{i g(z) \sigma_{3}} .
$$

$g(z)$ is chosen to satisfy the RHP 
- $g$ is analytic off $\Sigma^{\omega}=\left(\lambda_{L}\right.$ cut $) \cup\left(\lambda_{C}\right.$ cut $) \cup\left(\lambda_{R}\right.$ cut $)$,

- $g_{+}(z)+g_{-}(z)=\mathcal{G}\left(z ; \omega_{L}, \omega_{C}\right)= \begin{cases}\omega_{L}-i \ln \left(\delta^{2} / P_{-}\right), & \lambda_{L} \text { cut in the UHP, } \\ \omega_{L}-i \ln \left(\delta^{2} P_{-}\right), & \lambda_{L} \text { cut in the LHP, } \\ \omega_{C}-i \ln \left(\delta^{2} / P,\right) & \lambda_{C} \text { cut in the UHP, } \\ \omega_{C}-i \ln \left(\delta^{2} P\right), & \lambda_{C} \text { cut in the LHP, } \\ -i \ln \left(\delta^{2}\right), & \lambda_{R} \text { cut, }\end{cases}$

- $g \sim g(\infty)$ as $z \rightarrow \infty$,

for some real constant $g(\infty) . g(z)$ is given explicitly by

$$
g(z)=-\frac{R(z)}{2 \pi i} \int_{\Sigma^{\omega}} \frac{\mathcal{G}\left(\zeta ; \omega_{L}, \omega_{C}\right)}{(\zeta-z) R_{-}(\zeta)} \mathrm{d} \zeta
$$

where the integration is done along the branch cuts. $\omega_{L}$ and $\omega_{C}$ are chosen so $g(z) \sim g(\infty)$ for some constant $g(\infty)$ as $z \rightarrow \infty$. Specifically,

$$
\begin{aligned}
g(\infty)=\frac{1}{2 \pi i}\left[\int_{\Sigma^{\omega}} \frac{\mathcal{G}(\zeta)}{R(\zeta)} \zeta^{2} \mathrm{~d} \zeta-\Re(\alpha) \int_{\Sigma^{\omega}} \frac{\mathcal{G}(\zeta)}{R(\zeta)} \zeta \mathrm{d} \zeta\right. \\
\left.+\left(A^{2}-\frac{\mu^{2}}{4}+\frac{1}{2}[\Im(\alpha)]^{2}\right) \int_{\Sigma^{\omega}} \frac{\mathcal{G}(\zeta)}{R(\zeta)} \mathrm{d} \zeta\right]
\end{aligned}
$$

With this choice of $g$, the RHP for $M^{(6)}$ is formulated as

$$
P^{(6)}:\left\{\Sigma^{(6)}=\Sigma^{(5)}, V^{(6)}, e^{i(G(\infty) t+g(\infty)) \sigma_{3}} \text { as } z \rightarrow \infty\right\},
$$

where $V^{(6)}$ is defined by Figure 4.3 and

$$
\begin{array}{rlrl}
V_{1}^{(6)} & =\left[\begin{array}{cc}
1 & \delta^{2} P^{-1} e^{-i(h t+2 g)} \\
0 & 1
\end{array}\right], & V_{2}^{(6)} & =\left[\begin{array}{cc}
1 & 0 \\
\delta^{-2} P^{-1} e^{i(h t+2 g)} & 1
\end{array}\right], \\
V_{3}^{(6)} & =\left[\begin{array}{cc}
1 & 0 \\
\delta^{-2} \rho e^{i(h t+2 g)} & 1
\end{array}\right], & V_{4}^{(6)}=\left[\begin{array}{cc}
1 & \delta^{2} \rho e^{-i(h t+2 g)} \\
0 & 1
\end{array}\right], \\
V_{5}^{(6)}=\left[\begin{array}{cc}
1 & 0 \\
\delta^{-2} P e^{i(h t+2 g)} & 1
\end{array}\right], & V_{6}^{(6)}=\left[\begin{array}{cc}
1 & \delta^{2} P e^{-i(h t+2 g)} \\
0 & 1
\end{array}\right], \\
V_{7}^{(6)}=\left[\begin{array}{cc}
0 & e^{-i\left(\Omega_{C} t+\omega_{C}\right)} \\
-e^{i\left(\Omega_{C} t+\omega_{C}\right)} & 0
\end{array}\right], & V_{8}^{(6)}=\left[\begin{array}{ccc}
0 & -e^{-i\left(\Omega_{C} t+\omega_{C}\right)} \\
e^{i\left(\Omega_{C} t+\omega_{C}\right)} & 0
\end{array}\right], \\
V_{9}^{(6)}=\left[\begin{array}{ccc}
0 & e^{-i\left(\Omega_{L} t+\omega_{L}\right)} \\
-e^{i\left(\Omega_{L} t+\omega_{L}\right)} & 0
\end{array}\right], & V_{10}^{(6)}=\left[\begin{array}{cc}
0 & 1 \\
-1 & 0
\end{array}\right] .
\end{array}
$$

\subsection{The Model Problem $\boldsymbol{P}^{(\bmod )}$}

The model problem is defined by disregarding all but the three contours with constant jumps. Define $M^{(\mathrm{mod})}$ as the solution to the RHP

$$
P^{(\mathrm{mod})}:\left\{\Sigma^{(\mathrm{mod})}, V^{(\mathrm{mod})}, e^{i(G(\infty) t+g(\infty)) \sigma_{3}} \text { as } z \rightarrow \infty\right\} .
$$

Here

$$
\Sigma^{(\bmod )}=\left(\lambda_{L} \text { cut }\right) \cup\left(\lambda_{C} \text { cut }\right) \cup\left(\lambda_{R} \text { cut }\right),
$$




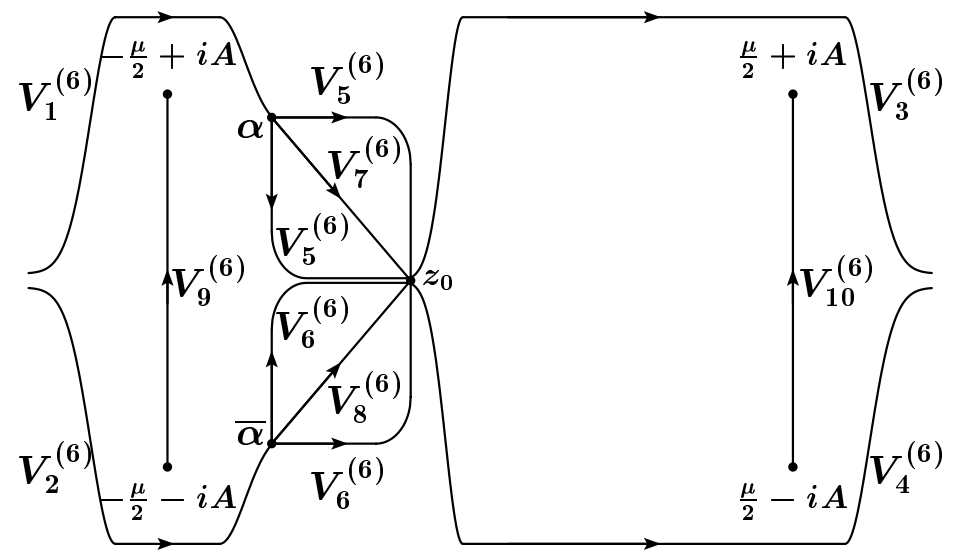

FIgURE 4.3. The RHP $P^{(6)}$ for the transition region.
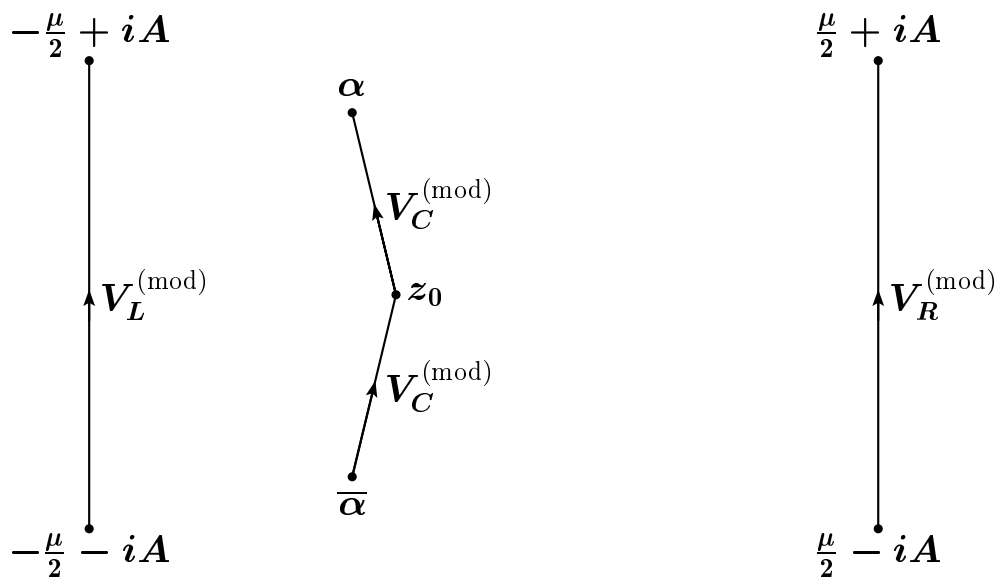

FIGURE 4.4. The RHP $P^{(\mathrm{mod})}$ for the transition region.

and the jump matrix $V^{(\mathrm{mod})}$ is given by Figure 4.4 and

$$
\begin{gathered}
V_{L}^{(\mathrm{mod})}=\left[\begin{array}{cc}
0 & e^{-i\left(\Omega_{L} t+\omega_{L}\right)} \\
-e^{i\left(\Omega_{L} t+\omega_{L}\right)} & 0
\end{array}\right], \\
V_{C}^{(\mathrm{mod})}=\left[\begin{array}{cc}
0 & -e^{-i\left(\Omega_{C} t+\omega_{C}\right)} \\
e^{i\left(\Omega_{C} t+\omega_{C}\right)} & 0
\end{array}\right], \quad V_{R}^{(\mathrm{mod})}=\left[\begin{array}{rr}
0 & 1 \\
-1 & 0
\end{array}\right] .
\end{gathered}
$$

We now write $M^{(6)}=M^{(\mathrm{err})} M^{(\mathrm{mod})}$ for large $z$, which gives, using equation (A.40),

$$
q(x, t)=-2\left(M_{1}^{(\mathrm{mod})}+M_{1}^{(\mathrm{err})}\right)_{12} e^{i\left[\left(G(\infty)-\mu|\xi|-\mu^{2} / 2+A^{2}\right) t+g(\infty)\right]} .
$$

Sections $4.6,4.7,4.8$, and 4.9 complete the error analysis by showing 


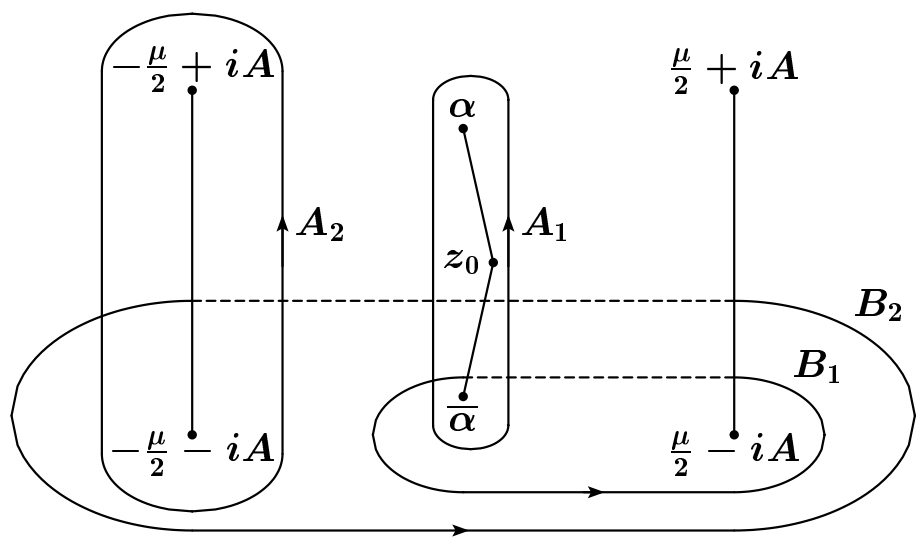

FIGURE 4.5. The canonical homology cycles $\left\{A_{1}, A_{2}, B_{1}, B_{2}\right\}$. The solid lines lie on the first sheet, while the dashed lines lie on the second sheet.

LEMMA 4.1 $\left|M_{1}^{(\mathrm{err})}\right|=O\left(t^{-1 / 2}\right)$.

The model problem is now solved explicitly in terms of theta functions. Let the cycles $A_{1}, A_{2}, B_{1}$, and $B_{2}$ define the canonical homology basis for the torus of genus 2 as shown in Figure 4.5. Now pick a basis of holomorphic differentials $\left\{\omega_{1}, \omega_{2}\right\}$ of the form

$$
\omega_{i}=\frac{P_{i}(z)}{\lambda_{L} \lambda_{C} \lambda_{R}} d z, \quad i=1,2,
$$

where $P_{i}(z)$ are first-order polynomials. Normalize the basis so

$$
\int_{A_{j}} \omega_{i}=1, \quad i, j=1,2 .
$$

The $2 \times 2$ Riemann period matrix is given by

$$
\tau_{i j}=\int_{B_{j}} \omega_{i}, \quad i, j=1,2 .
$$

$\tau$ is purely imaginary and symmetric, and $-i \tau$ is positive definite. Define the theta function by

$$
\theta(s)=\sum_{\ell \in \mathbb{Z}^{2}} e^{2 \pi i\langle\ell, s\rangle+\pi i\langle\ell, \tau \ell\rangle}, \quad s \in \mathbb{C}^{2},
$$


where $\langle\cdot, \cdot\rangle$ is the standard inner product for $\mathbb{C}^{2}$. Define $e_{i}$ as the $i^{\text {th }}$ column of $\left[\begin{array}{ll}1 & 0 \\ 0 & 1\end{array}\right]$ and $s_{i}$ as the $i^{\text {th }}$ entry of $s$. Then $\theta(s)$ has the properties

$$
\begin{aligned}
\theta(s) & =\theta(-s), \\
\theta\left(s+e_{i}\right) & =\theta(s), \\
\theta\left(s+\tau e_{i}\right) & =e^{2 \pi i s_{i}-\pi i \tau_{i i}} \theta(s) .
\end{aligned}
$$

The vector-valued function $u(z)$ is given by

$$
u(z)=\left(\int_{\mu / 2-i A}^{z} \omega_{1}, \quad \int_{\mu / 2-i A}^{z} \omega_{2}\right)^{\top} .
$$

Let

$$
\begin{aligned}
& \Omega=\left(\Omega_{L}, \Omega_{C}\right)^{\top}, \\
& \omega=\left(\omega_{L}, \omega_{C}\right)^{\top},
\end{aligned}
$$

and define the single-valued function

$$
\begin{aligned}
\mathcal{M}(z, d) & =\left(\mathcal{M}_{1}, \mathcal{M}_{2}\right) \\
& =\left(\frac{\theta\left(\frac{\Omega}{2 \pi} t+\frac{\omega}{2 \pi}+u(z)+d\right)}{\theta(u(z)+d)}, \frac{\theta\left(\frac{\Omega}{2 \pi} t+\frac{\omega}{2 \pi}-u(z)+d\right)}{\theta(-u(z)+d)}\right),
\end{aligned}
$$

where $d \in \mathbb{C}^{2}$ is determined below. The jump conditions for $\mathcal{M}$ are given by

$$
\begin{aligned}
& \mathcal{M}_{+}=\mathcal{M}_{-}\left[\begin{array}{cc}
0 & e^{-i\left(\Omega_{L} t+\omega_{L}\right)} \\
e^{i\left(\Omega_{L} t+\omega_{L}\right)} & 0
\end{array}\right] \quad \text { on the } \lambda_{L} \text { cut } \\
& \mathcal{M}_{+}=\mathcal{M}_{-}\left[\begin{array}{cc}
0 & e^{-i\left(\Omega_{C} t+\omega_{C}\right)} \\
e^{i\left(\Omega_{C} t+\omega_{C}\right)} & 0
\end{array}\right] \text { on the } \lambda_{C} \text { cut } \\
& \mathcal{M}_{+}=\mathcal{M}_{-}\left[\begin{array}{ll}
0 & 1 \\
1 & 0
\end{array}\right] \quad \text { on the } \lambda_{R} \text { cut. }
\end{aligned}
$$

Next, define

$$
\gamma(z)=\left(\frac{\left(z-\left(-\frac{\mu}{2}+i A\right)\right) \cdot(z-\alpha) \cdot\left(z-\left(\frac{\mu}{2}+i A\right)\right)}{\left(z-\left(-\frac{\mu}{2}-i A\right)\right) \cdot(z-\bar{\alpha}) \cdot\left(z-\left(\frac{\mu}{2}-i A\right)\right)}\right)^{1 / 4}
$$

so that $\gamma(z) \sim 1$ as $z \rightarrow \infty$ and the branch cuts lie on the $\lambda_{L}, \lambda_{C}$, and $\lambda_{R}$ cuts. Also set

$$
\mathcal{N}=\frac{1}{2}\left[\begin{array}{cc}
\left(\gamma+\gamma^{-1}\right) \mathcal{M}_{1}(z, d) & -i\left(\gamma-\gamma^{-1}\right) \mathcal{M}_{2}(z, d) \\
i\left(\gamma-\gamma^{-1}\right) \mathcal{M}_{1}(z,-d) & \left(\gamma+\gamma^{-1}\right) \mathcal{M}_{2}(z,-d)
\end{array}\right]
$$


$\mathcal{N}$ has the correct jump conditions:

$$
\begin{aligned}
& \mathcal{N}_{+}=\mathcal{N}_{-}\left[\begin{array}{cc}
0 & e^{-i\left(\Omega_{L} t+\omega_{L}\right)} \\
-e^{i\left(\Omega_{L} t+\omega_{L}\right)} & 0
\end{array}\right] \quad \text { on the } \lambda_{L} \text { cut, } \\
& \mathcal{N}_{+}=\mathcal{N}_{-}\left[\begin{array}{cc}
0 & e^{-i\left(\Omega_{C} t+\omega_{C}\right)} \\
-e^{i\left(\Omega_{C} t+\omega_{C}\right)} & 0
\end{array}\right] \text { on the } \lambda_{C} \text { cut } \\
& \mathcal{N}_{+}=\mathcal{N}_{-}\left[\begin{array}{rr}
0 & 1 \\
-1 & 0
\end{array}\right] \quad \text { on the } \lambda_{R} \text { cut. }
\end{aligned}
$$

Let $z_{1}$ and $z_{2}$ be the two zeros of $\gamma(z)-\gamma^{-1}(z)$ and take

$$
d=-\int_{\bar{\alpha}}^{X_{2}\left(z_{1}\right)}\left(\omega_{1}, \omega_{2}\right)^{\top}-\int_{-\mu / 2-i A}^{X_{2}\left(z_{2}\right)}\left(\omega_{1}, \omega_{2}\right)^{\top},
$$

where $X\left(z_{i}\right)$ is the preimage of $z_{i}$ on the second sheet. By Farkas and Kra [11], $X_{1}\left(z_{1}\right)$ and $X_{1}\left(z_{2}\right)$ are the two zeros of $\theta(u(z)-d)$, so $\mathcal{N}$ is analytic off the branch cuts. Now the solution to the model problem is given by

$$
M^{(\bmod )}(z)=e^{i(G(\infty) t+g(\infty)) \sigma_{3}} \mathcal{N}^{-1}(\infty) \mathcal{N}(z) .
$$

Now

$$
\gamma(z)-\gamma^{-1}(z)=-i(2 A+\Im(\alpha)) z^{-1}+O\left(z^{-2}\right) \quad \text { as } z \rightarrow \infty
$$

and

$$
\mathcal{N}^{-1}(\infty)=\left[\begin{array}{cc}
\frac{1}{\mathcal{M}_{1}(\infty, d)} & 0 \\
0 & \frac{1}{\mathcal{M}_{2}(\infty,-d)}
\end{array}\right] .
$$

Therefore from equation (4.41),

$$
\left(M_{1}^{(\mathrm{mod})}\right)_{12}=-\frac{1}{2}(2 A+\Im(\alpha)) \frac{\mathcal{M}_{2}(\infty, d)}{\mathcal{M}_{1}(\infty, d)} e^{i[G(\infty) t+g(\infty)]} .
$$

\subsection{The Error Problem $P^{(\mathrm{err})}$}

The jump matrices off $\Sigma^{(\bmod )}$ decay uniformly in time to the identity outside of neighborhoods of $z_{0}, \alpha$, and $\bar{\alpha}$. Take $r_{\alpha}^{\varepsilon}, r_{\bar{\alpha}}^{\varepsilon}$, and $r_{z_{0}}^{\varepsilon}$ to be small circles of radius $\varepsilon$ centered around $\alpha, \bar{\alpha}$, and $z_{0}$, respectively, with $\varepsilon$ chosen so the circles do not intersect each other or the $\lambda_{L}$ or $\lambda_{R}$ branch cuts. Write

$$
M^{(6)}=M^{(\mathrm{err})} M^{(\mathrm{app})}
$$

everywhere in the complex plane. $M^{(\mathrm{app})}$ is defined as

$$
M^{(\mathrm{app})}= \begin{cases}\text { parametrix of } V^{(6)} & \text { inside } r_{\alpha}^{\varepsilon}, r_{\bar{\alpha}}^{\varepsilon}, r_{z_{0}}^{\varepsilon}, \\ M^{(\mathrm{mod})} & \text { outside } r_{\alpha}^{\varepsilon}, r_{\bar{\alpha}}^{\varepsilon}, r_{z_{0}}^{\varepsilon},\end{cases}
$$

and satisfies the RHP

$$
P^{(\mathrm{app})}:\left\{\Sigma^{(\mathrm{app})}, V^{(\mathrm{app})}, e^{i(G(\infty) t+g(\infty)) \sigma_{3}} \text { as } z \rightarrow \infty\right\} .
$$



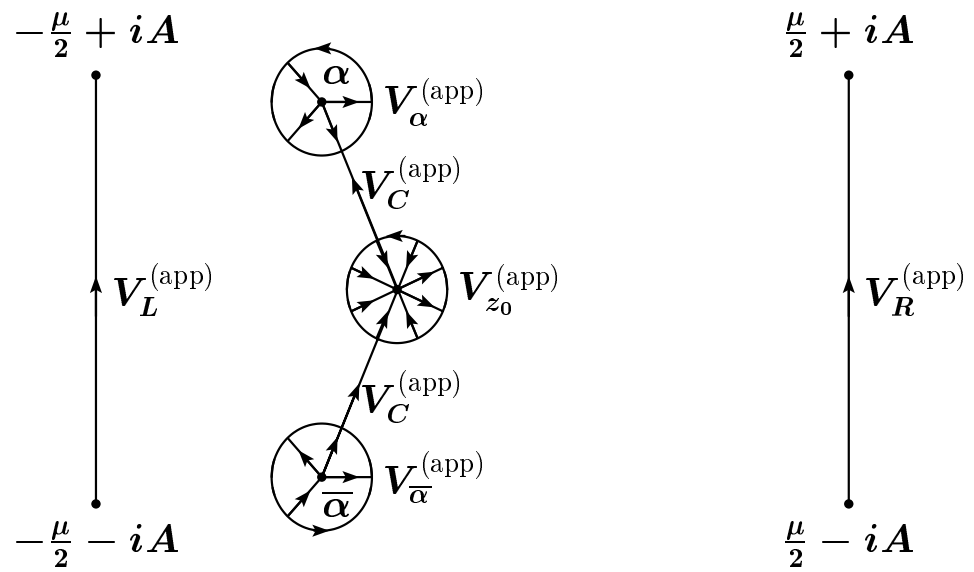

FIGURE 4.6. The RHP $P^{(\mathrm{app})}$ for the transition region.
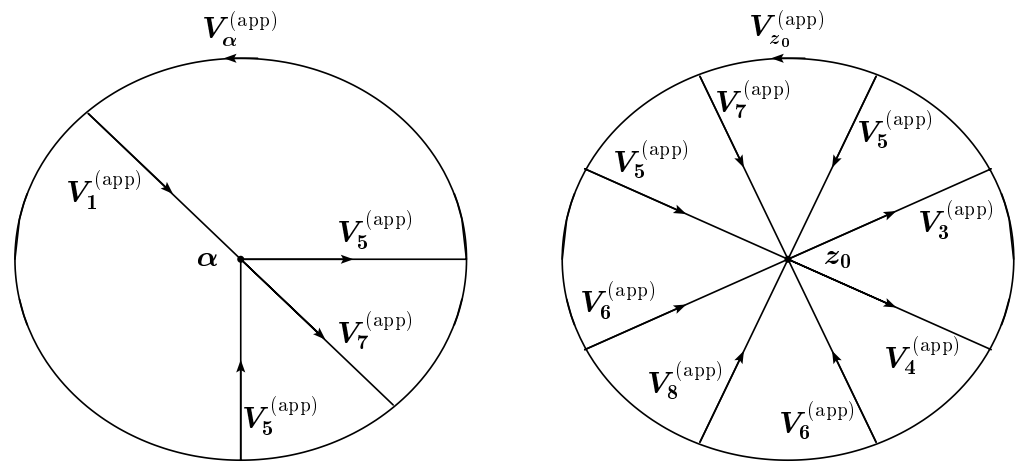

FIGURE 4.7. Detail of the RHP $P^{(\mathrm{app})}$ inside $r_{z_{0}}^{\alpha}$ and $r_{z_{0}}^{\varepsilon}$.

See Figures 4.6 and 4.7. The jump $V^{(\mathrm{app})}$ is given by

$$
\begin{gathered}
V_{L}^{(\mathrm{app})}=V_{L}^{(\mathrm{mod})}, \quad V_{C}^{(\mathrm{app})}=V_{C}^{(\mathrm{mod})}, \quad V_{R}^{(\mathrm{app})}=V_{R}^{(\mathrm{mod})}, \\
V_{i}^{(\mathrm{app})}=V_{i}^{(6)}, \quad i=1, \ldots, 8 .
\end{gathered}
$$

$V_{z_{0}}^{(\text {app })}$ has the form $I+O\left(t^{-1 / 2}\right)$, and $V_{\alpha}^{(\text {app })}$ and $V_{\bar{\alpha}}^{(\text {app })}$ have the form $I+O\left(t^{-1}\right)$. This definition of $M^{(\mathrm{app})}$ implies $M^{(\mathrm{err})}$ satisfies the RHP

$$
P^{(\mathrm{err})}:\left\{\Sigma^{(\mathrm{err})}, V^{(\mathrm{err})}, I \text { as } z \rightarrow \infty\right\}
$$




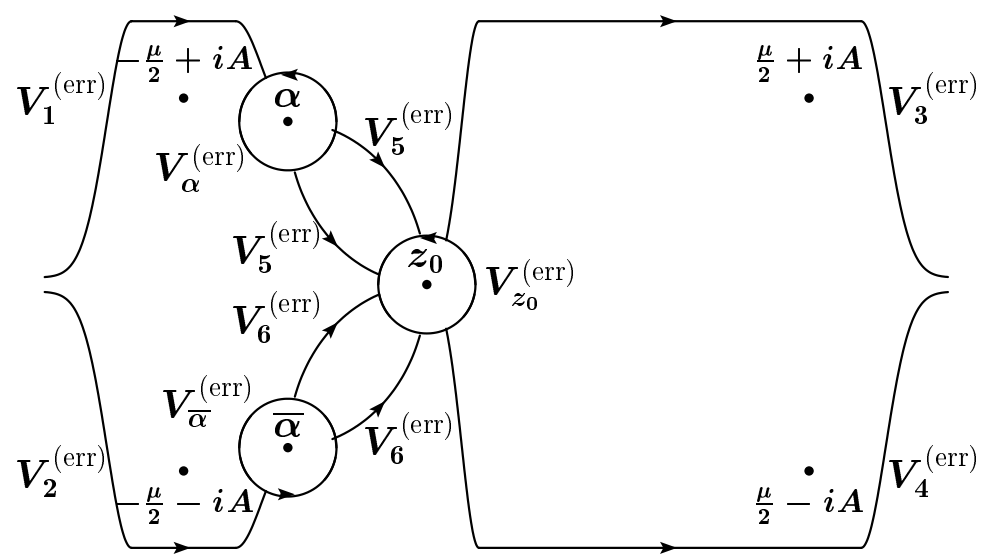

FIGURE 4.8. The RHP $P^{(\mathrm{err})}$ for the transition region.

where $V^{(\mathrm{err})}$ is defined by Figure 4.8 and

$$
\begin{aligned}
& V_{i}^{(\mathrm{err})}=M^{(\mathrm{app})} V_{i}^{(6)}\left(M^{(\mathrm{app})}\right)^{-1} \quad \text { outside } r_{z_{0}}^{\varepsilon}, r_{\alpha}^{\varepsilon} \text {, and } r_{\bar{\alpha}}^{\varepsilon}, i=1, \ldots, 6 \text {, } \\
& V_{z_{0}}^{(\mathrm{err})}=M_{-}^{(\mathrm{app})}\left(V_{z_{0}}^{(\mathrm{app})}\right)^{-1}\left(M_{-}^{(\mathrm{app})}\right)^{-1} \text { on } r_{z_{0}}^{\varepsilon} \text {, } \\
& V_{\alpha}^{(\mathrm{err})}=M_{-}^{(\mathrm{app})}\left(V_{\alpha}^{(\mathrm{app})}\right)^{-1}\left(M_{-}^{(\mathrm{app})}\right)^{-1} \text { on } r_{\alpha}^{\varepsilon} \text {, } \\
& V_{\bar{\alpha}}^{(\mathrm{err})}=M_{-}^{(\mathrm{app})}\left(V_{\bar{\alpha}}^{(\mathrm{app})}\right)^{-1}\left(M_{-}^{(\mathrm{app})}\right)^{-1} \text { on } r_{\bar{\alpha}}^{\varepsilon} \text {. }
\end{aligned}
$$

\subsection{Construction of the Parametrix near $\alpha$ Using Airy Functions}

The parametrix near $\alpha$ will be constructed explicitly using the method of Deift, Kriecherbauer, McLaughlin, Venakides, and Zhou [5]. The construction around $\bar{\alpha}$ is similar. Start with the RHP

$$
P^{(Q 0)}:\left\{\Sigma^{(Q 0)}, V^{(Q 0)}, M^{(\bmod )} \text { on } r_{\alpha}^{\varepsilon}\right\} .
$$

The jump matrices $V_{i}^{(Q 0)}$ are given by Figure 4.9 and

$$
\begin{gathered}
V_{1}^{(Q 0)}=\left[\begin{array}{cc}
1 & \delta^{2} P^{-1} e^{-i(h t+2 g)} \\
0 & 1
\end{array}\right], \quad V_{2}^{(Q 0)}=\left[\begin{array}{cc}
1 & 0 \\
\delta^{-2} P e^{i(h t+2 g)} & 1
\end{array}\right], \\
V_{3}^{(Q 0)}=\left[\begin{array}{cc}
0 & \delta^{2} P^{-1} e^{-i\left[\left(f+G_{+}+G_{-}\right) t+g_{+}+g_{-}\right]} \\
-\delta^{-2} P e^{i\left[\left(f+G_{+}+G_{-}\right) t+g_{+}+g_{-}\right]} & 0
\end{array}\right] .
\end{gathered}
$$

The jump matrices can be reduced to constants by writing

$$
V^{(Q 1)}=e^{-i\left[\frac{i}{2} \ln \left(\delta^{2} / P\right)+\left(\frac{1}{2} f+G_{-}\right) t+g_{-}\right] \sigma_{3}} V^{(Q 0)} e^{i\left[\frac{i}{2} \ln \left(\delta^{2} / P\right)+\left(\frac{1}{2} f+G_{+}\right) t+g_{+}\right] \sigma_{3}}
$$

or

$$
V_{1}^{(Q 1)}=\left[\begin{array}{ll}
1 & 1 \\
0 & 1
\end{array}\right], \quad V_{2}^{(Q 1)}=\left[\begin{array}{ll}
1 & 0 \\
1 & 1
\end{array}\right], \quad V_{3}^{(Q 1)}=\left[\begin{array}{rr}
0 & 1 \\
-1 & 0
\end{array}\right]
$$


The function $Q^{(1)}$ satisfies the new RHP $P^{(Q 1)}$. Now introduce the auxiliary contour $\Sigma^{(Q 1)}$ that divides the complex plane into four regions, I, II, III, and IV (see Figure 4.9). The function

$$
Q^{(1)}(\zeta)=\left\{\begin{array}{lc}
{\left[\begin{array}{cc}
\operatorname{Ai}(\zeta) & \operatorname{Ai}\left(e^{4 i \pi / 3} \zeta\right) \\
\operatorname{Ai}^{\prime}(\zeta) & e^{4 i \pi / 3} \operatorname{Ai}^{\prime}\left(e^{4 i \pi / 3} \zeta\right)
\end{array}\right] e^{-i \pi \sigma_{3} / 6},} & \\
{\left[\begin{array}{cc}
\operatorname{Ai}(\zeta) & \operatorname{Ai}\left(e^{4 i \pi / 3} \zeta\right) \\
\operatorname{Ai}^{\prime}(\zeta) & e^{4 i \pi / 3} \operatorname{Ai}^{\prime}\left(e^{4 i \pi / 3} \zeta\right)
\end{array}\right] e^{-i \pi \sigma_{3} / 6}\left[\begin{array}{rr}
1 & 0 \\
-1 & 1
\end{array}\right],} & \zeta \in \mathrm{II}, \\
{\left[\begin{array}{cc}
\operatorname{Ai}(\zeta) & -e^{4 i \pi / 3} \operatorname{Ai}\left(e^{2 i \pi / 3} \zeta\right) \\
\operatorname{Ai}^{\prime}(\zeta) & -\operatorname{Ai}^{\prime}\left(e^{2 i \pi / 3} \zeta\right)
\end{array}\right] e^{-i \pi \sigma_{3} / 6}\left[\begin{array}{cc}
1 & 0 \\
1 & 1
\end{array}\right],} & \zeta \in \mathrm{III}, \\
{\left[\begin{array}{cc}
\mathrm{Ai}(\zeta) & -e^{4 i \pi / 3} \operatorname{Ai}\left(e^{2 i \pi / 3} \zeta\right) \\
\operatorname{Ai}^{\prime}(\zeta) & -\operatorname{Ai}^{\prime}\left(e^{2 i \pi / 3} \zeta\right)
\end{array}\right] e^{-i \pi \sigma_{3} / 6},} & \zeta \in \mathrm{IV},
\end{array}\right.
$$

satisfies the jump conditions $Q_{+}^{(1)}=Q_{-}^{(1)} V_{i}^{(Q 1)}$ on $\Sigma^{(Q 1)}$, which can be checked with the aid of the identity

$$
\operatorname{Ai}(\zeta)+e^{2 i \pi / 3} \operatorname{Ai}\left(e^{2 i \pi / 3} \zeta\right)+e^{4 i \pi / 3} \operatorname{Ai}\left(e^{4 i \pi / 3} \zeta\right)=0 .
$$

Now suppose $F(z): r_{\alpha}^{\varepsilon} \rightarrow F\left(r_{\alpha}^{\varepsilon}\right)$ is a biholomorphic function mapping $\Sigma^{(Q 0)} \cap r_{\alpha}^{\varepsilon}$ onto $\Sigma^{(Q 1)} \cap F\left(r_{\alpha}^{\varepsilon}\right)$ and satisfying $F(\alpha)=0$. Then, for an appropriate choice of the analytic $2 \times 2$ matrix-valued function $E(z)$,

$$
Q^{(0)}(z)=E(z) Q^{(1)}(F(z)) e^{i\left[\frac{i}{2} \ln \left(\delta^{2} / P\right)+\left(\frac{1}{2} f+G\right) t+g\right] \sigma_{3}}
$$

will satisfy the RHP $\left(V^{(Q 0)}, \Sigma^{(Q 0)}\right) . E(z)$ and $F(z)$ are chosen using the asymptotics of the Airy function (see Abramowitz and Stegun [2]):

$$
\begin{aligned}
\operatorname{Ai}(\zeta) \sim \frac{1}{2 \sqrt{\pi}} \zeta^{-\frac{1}{4}} e^{-\frac{2}{3} \zeta^{3 / 2}}\left(1+O\left(\zeta^{-3 / 2}\right)\right), & |\arg (\zeta)<\pi| \\
\operatorname{Ai}^{\prime}(\zeta) \sim-\frac{1}{2 \sqrt{\pi}} \zeta^{\frac{1}{4}} e^{-\frac{2}{3} \zeta^{3 / 2}}\left(1+O\left(\zeta^{-3 / 2}\right)\right), & |\arg (\zeta)<\pi| .
\end{aligned}
$$

The asymptotics of Ai show that $F(z)$ should satisfy

$$
\frac{2}{3}(F(z))^{3 / 2}=i(z-\alpha)^{3 / 2} \mathcal{H}(z) t
$$

where $\mathcal{H}(z)$ is analytic. Therefore,

$$
F(z)=(z-\alpha)\left(\frac{3 i}{2} \mathcal{H} t\right)^{3 / 2}
$$

is analytic. Now $E(z)$ is chosen to be an analytic approximation of

$$
M^{(\mathrm{mod})}\left[Q^{(1)}(F(z)) e^{i\left[\frac{i}{2} \ln \left(\delta^{2} / P\right)+\left(\frac{1}{2} f+G\right) t+g\right] \sigma_{3}}\right]^{-1} .
$$




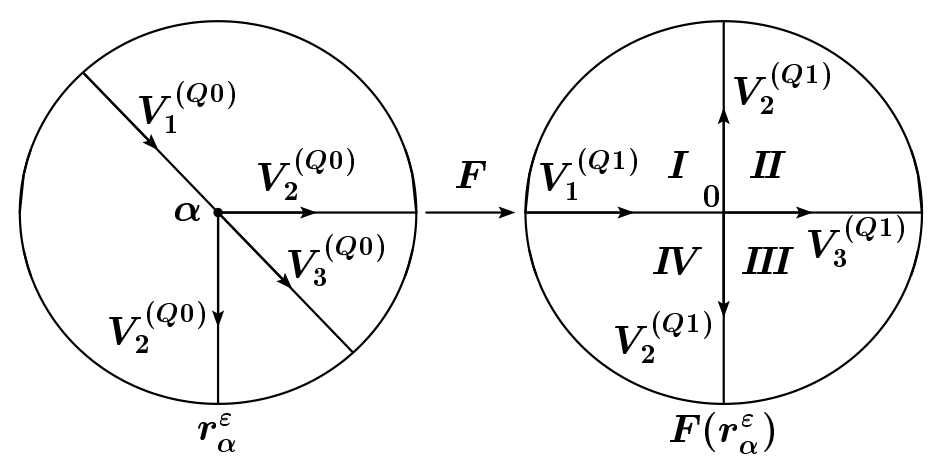

FIGURE 4.9. The RHPs $P^{(\mathrm{Q} 0)}$ and $P^{(\mathrm{Q} 1)}$.

From equations (4.55) and (4.56),

$$
Q^{(1)}(\zeta) e^{u \sigma_{3}} \sim \frac{e^{i \pi / 12}}{2 \sqrt{\pi}}\left[\begin{array}{cc}
\zeta^{-1 / 4} & 0 \\
0 & \zeta^{1 / 4}
\end{array}\right]\left(\left[\begin{array}{rr}
1 & 1 \\
-1 & 1
\end{array}\right] e^{-i \pi \sigma_{3} / 4}+O\left(u^{-1}\right)\right)
$$

Choose

$$
E(z)=\sqrt{\pi} e^{-i \pi / 12} M^{(\bmod )}(z) e^{i \pi \sigma_{3} / 4}\left[\begin{array}{rr}
1 & -1 \\
1 & 1
\end{array}\right]\left[\begin{array}{cc}
(F(z))^{1 / 4} & 0 \\
0 & (F(z))^{-1 / 4}
\end{array}\right] .
$$

This gives

$$
V_{\alpha}^{(\mathrm{app})}=I+O\left(t^{-1}\right)
$$

and completes the construction of the parametrix $Q^{(0)}(z)$.

\subsection{Construction of the Parametrix near $z_{0}$}

The construction of the parametrix near $z_{0}$ is similar to the construction in the plane-wave and residual regions with additional contour splits. See Appendix B for details in the plane-wave case. However, in the transition region extra care must be taken because $z_{0}$ now lies on the $\lambda_{C}$ cut, which is a branch cut of $h(z)$.

To the left of the $\lambda_{C}$ cut and in $D_{z_{0}}^{\varepsilon}, h(z)$ can be written in a Taylor series as

$$
h(z)=h_{0}^{L}+\sum_{n=2}^{\infty} h_{n}^{L}\left(z-z_{0}\right)^{n} .
$$

Similarly, to the right of the $\lambda_{C}$ cut and in $D_{z_{0}}^{\varepsilon}, h(z)$ can be written

$$
h(z)=h_{0}^{R}+\sum_{n=2}^{\infty} h_{n}^{R}\left(z-z_{0}\right)^{n} .
$$




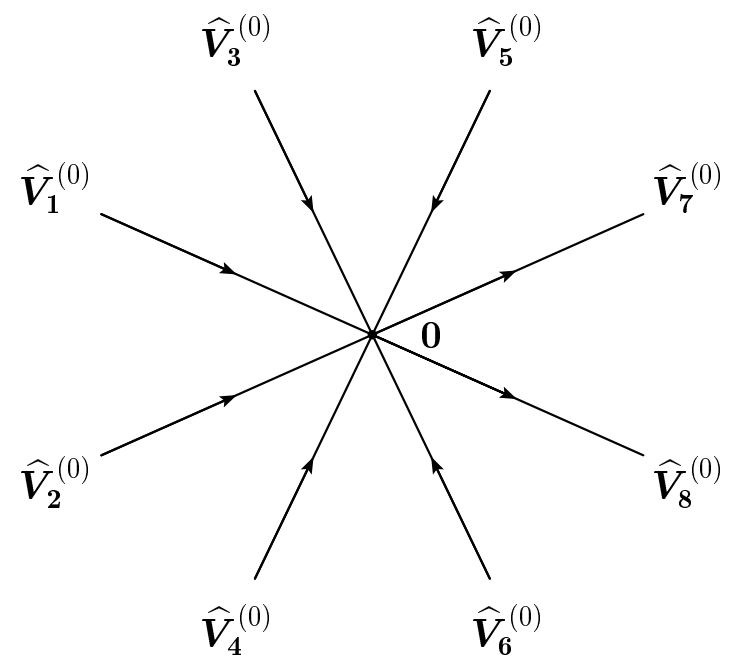

FIGURE 4.10. The RHP $\widehat{P}^{(0)}$ for the transition region.

In $D_{z 0}^{\varepsilon}$, define $T: z \rightarrow \hat{z}$ by

$$
\hat{z}= \begin{cases}i \sqrt{\frac{t}{2}}\left(z-z_{0}\right)\left(\sum_{n=0}^{\infty} h_{n+2}^{L}\left(z-z_{0}\right)^{n}\right)^{1 / 2} & \text { left of the } \lambda_{C} \text { cut } \\ \sqrt{\frac{t}{2}}\left(z-z_{0}\right)\left(\sum_{n=0}^{\infty} h_{n+2}^{R}\left(z-z_{0}\right)^{n}\right)^{1 / 2} & \text { right of the } \lambda_{C} \text { cut. }\end{cases}
$$

Now

$$
T(h(z) t)= \begin{cases}h_{0}^{L} t-2 \hat{z}^{2} & \text { left of the } \lambda_{C} \text { cut, } \\ h_{0}^{R} t+2 \hat{z}^{2} & \text { right of the } \lambda_{C} \text { cut. }\end{cases}
$$

The transformation is chosen so that a $z$ on the $\lambda_{C}$ cut is mapped to the same $\hat{z}$ using either formula.

In $T\left(D_{z_{0}}^{\varepsilon}\right), \widehat{M}^{(0)}$ is defined to satisfy the jump conditions of $T\left(V^{(6)}\right) . \widehat{\Sigma}^{(0)}$ is chosen to be four straight lines through $\hat{z}=0$ that do not intersect $T\left(\lambda_{L}\right.$ cut) or $T\left(\lambda_{R}\right.$ cut) (see Figure B.1). As in Appendix B, we can express $z$ in terms of $\hat{z}$ as

$$
z=z_{0}+\sum_{n=1}^{\infty} \beta_{n}\left(\frac{\hat{z}}{\sqrt{t}}\right)^{n}
$$

where the $\beta_{n}$ can be expressed in terms of known quantities and are understood to be possibly different if $\hat{z}$ is to the left or right of $T\left(\lambda_{C}\right.$ cut). Given $F(z)$, define $\widehat{F}$ by

$$
\widehat{F}(\hat{z})=F\left(z_{0}+\sum_{n=1}^{\infty} \beta_{n}\left(\frac{\hat{z}}{\sqrt{t}}\right)^{n}\right) .
$$


The RHP is written as

$$
\widehat{P}^{(0)}:\left\{\widehat{\Sigma}^{(0)}, \widehat{V}^{(0)}, I \text { as } \hat{z} \rightarrow \infty\right\},
$$

with

$$
\begin{array}{rlrl}
\widehat{V}_{1}^{(0)} & =\left[\begin{array}{cc}
1 & 0 \\
\hat{\delta}^{-2} \widehat{P} e^{i\left(h_{0}^{L} t-2 \hat{z}^{2}+2 \widehat{g}\right)} & 1
\end{array}\right], & \widehat{V}_{2}^{(0)}=\left[\begin{array}{cc}
1 & \hat{\delta}^{2} \widehat{P} e^{-i\left(h_{0}^{L} t-2 \hat{z}^{2}+2 \widehat{g}\right)} \\
0 & 1
\end{array}\right], \\
\widehat{V}_{3}^{(0)}=\left[\begin{array}{cc}
0 & e^{-i\left(\Omega_{C} t+\omega_{C}\right)} \\
-e^{i\left(\Omega_{C} t+\omega_{C}\right)} & 0
\end{array}\right], & \widehat{V}_{4}^{(0)}=\left[\begin{array}{cc}
0 & -e^{-i\left(\Omega_{C} t+\omega_{C}\right)} \\
e^{i\left(\Omega_{C} t+\omega_{C}\right)} & 0
\end{array}\right], \\
\widehat{V}_{5}^{(0)}=\left[\begin{array}{cc}
1 & 0 \\
\hat{\delta}^{-2} \widehat{P} e^{i\left(h_{0}^{R} t+2 \hat{z}^{2}+2 \widehat{g}\right)} & 1
\end{array}\right], & \widehat{V}_{6}^{(0)}=\left[\begin{array}{cc}
1 & \hat{\delta}^{2} \widehat{P} e^{-i\left(h_{0}^{R} t+2 \hat{z}^{2}+2 \widehat{g}\right)} \\
0 & 1
\end{array}\right], \\
\widehat{V}_{7}^{(0)}=\left[\begin{array}{cc}
1 & 0 \\
\hat{\delta}^{-2} \hat{\rho} e^{i\left(h_{0}^{R} t+2 \hat{z}^{2}+2 \widehat{g}\right)} & 1
\end{array}\right], & \widehat{V}_{8}^{(0)}=\left[\begin{array}{cc}
1 & \hat{\delta}^{2} \hat{\rho} e^{-i\left(h_{0}^{R} t+2 \hat{z}^{2}+2 \widehat{g}\right)} \\
0 & 1
\end{array}\right] .
\end{array}
$$

The construction of the parametrix now continues along similar lines as in Appendix B, yielding

$$
V_{z 0}^{(\mathrm{app})}=I+O\left(t^{-1 / 2}\right) .
$$

\subsection{The Error Estimate}

Combining equations (4.48), (4.61), and (4.69) and the uniform decay of $V_{i}^{(\mathrm{err})}$, $i=1, \ldots, 6$, gives

$$
\left\|V^{(\mathrm{err})}-I\right\|_{L^{p}\left(\Sigma^{(\mathrm{err})}\right)}=O\left(t^{-1 / 2}\right), \quad p=1,2 .
$$

Continuing as in the error estimate for the plane-wave and residual regions leads to $\left|M_{1}^{(\mathrm{err})}\right|=O\left(t^{-1 / 2}\right)$, which is Lemma 4.1. Now Theorem 1.3 is proven by Lemma 4.1 and equations (4.24) and (4.44).

\section{Appendix A: The Forward Scattering Problem}

\section{A.1 Solution to the Zakharov-Shabat Eigenvalue Problem at $\boldsymbol{t}=\mathbf{0}$}

For any time $t$ and large enough $x, q(x, t)$ should behave asymptotically like a plane wave $A e^{-i \mu|x|-i k t}$. The dispersion relation from NLS is $k=\mu^{2} / 2-A^{2}$, so $q(x, t) \sim A e^{-i \mu|x|-i\left(\mu^{2} / 2-A^{2}\right) t}$ as $|x| \rightarrow \infty$. Start with the Lax pair (1.3)-(1.4) and make the transformation

$$
W(x, t)=\left[\begin{array}{cc}
e^{-i\left[\mu|x|+\left(\mu^{2} / 2-A^{2}\right) t\right] / 2} & 0 \\
0 & e^{i\left[\mu|x|+\left(\mu^{2} / 2-A^{2}\right) t\right] / 2}
\end{array}\right] U(x, t) .
$$

The Lax pair becomes

$$
\text { (A.2) } U_{x}=-i\left[\begin{array}{cc}
\left(z \mp \frac{\mu}{2}\right) & q e^{i \mu|x|+i\left(\mu^{2} / 2-A^{2}\right) t} \\
\bar{q} e^{-i \mu|x|-i\left(\frac{\mu^{2}}{2}-A^{2}\right) t} & -\left(z \mp \frac{\mu}{2}\right)
\end{array}\right] U=-i \widehat{D} U \text {, }
$$


(A.3)

$$
\begin{aligned}
U_{t} & =-i\left[\begin{array}{cc}
z^{2}-\frac{1}{2}|q|^{2}-\frac{1}{2}\left(\frac{\mu^{2}}{2}-A^{2}\right) & \left(z q+\frac{1}{2} i q_{x}\right) e^{i\left[\mu|x|+\left(\mu^{2} / 2-A^{2}\right) t\right]} \\
\left(z \bar{q}-\frac{1}{2} i \bar{q}_{x}\right) e^{-i\left[\mu|x|+\left(\mu^{2} / 2-A^{2}\right) t\right]} & -z^{2}+\frac{1}{2}|q|^{2}+\frac{1}{2}\left(\mu^{2} / 2-A^{2}\right)
\end{array}\right] U \\
& =-i \widehat{C} U
\end{aligned}
$$

where the minus sign holds for $x>0$ and the plus sign for $x<0$. At time $t=0$, the $x$-evolution equation simplifies to

$$
U_{x}=-i\left[\begin{array}{cc}
\left(z \mp \frac{\mu}{2}\right) & A \\
A & -\left(z \mp \frac{\mu}{2}\right)
\end{array}\right] U \text {. }
$$

This linear system can be solved explicitly for $U$. The matching condition between the solution for $x>0$ and $x<0$ is that $U(x)$ is continuous at $x=0$. For $z \in \mathbb{R}$,

$$
U(x, z)= \begin{cases}{\left[\begin{array}{cc}
\frac{-\lambda_{L}+(z+\mu / 2)}{A} & 1 \\
1 & \frac{\lambda_{L}-(z+\mu / 2)}{A}
\end{array}\right]\left[\begin{array}{cc}
e^{i \lambda_{L} x} & 0 \\
0 & e^{-i \lambda_{L} x}
\end{array}\right]\left[\begin{array}{l}
c_{1 L} \\
c_{2 L}
\end{array}\right],} & x<0, \\
{\left[\begin{array}{cc}
\frac{-\lambda_{R}+(z-\mu / 2)}{A} & 1 \\
1 & \frac{\lambda_{R}-(z-\mu / 2)}{A}
\end{array}\right]\left[\begin{array}{cc}
e^{i \lambda_{R} x} & 0 \\
0 & e^{-i \lambda_{R} x}
\end{array}\right]\left[\begin{array}{l}
c_{1 R} \\
c_{2 R}
\end{array}\right],} & x>0 .\end{cases}
$$

Here $c_{1 L}, c_{2 L}, c_{1 R}$, and $c_{2 R}$ are constants independent of $x$ that may depend on $z$. $\lambda_{L}$ and $\lambda_{R}$ are defined by (1.9).

\section{A.2 The Reflection Coefficient at $t=0$}

The eigenvector solutions $\Psi_{1}$ and $\Psi_{2}$ (see [3]) are defined for real $z$ by their behavior as $x \rightarrow \infty$ :

$$
\Psi_{1}(z ; x, t)=\left[\begin{array}{c}
1 \\
\frac{\lambda_{R}-(z-\mu / 2)}{A}
\end{array}\right] e^{-i \lambda_{R} x}, \quad \Psi_{2}(z ; x, t)=\left[\begin{array}{c}
\frac{-\lambda_{R}+(z-\mu / 2)}{A} \\
1
\end{array}\right] e^{i \lambda_{R} x} .
$$

Similarly, let $\Phi_{1}$ and $\Phi_{2}$ be defined by their behavior as $x \rightarrow-\infty$ :

$$
\Phi_{1}(z ; x, t)=\left[\begin{array}{c}
1 \\
\frac{\lambda_{L}-(z+\mu / 2)}{A}
\end{array}\right] e^{-i \lambda_{L} x}, \quad \Phi_{2}(z ; x, t)=\left[\begin{array}{c}
\frac{-\lambda_{L}+(z+\mu / 2)}{A} \\
1
\end{array}\right] e^{i \lambda_{L} x} .
$$

The initial data is symmetric in $x$, so from here on the problem is restricted to $x \geq 0 . \Psi_{1}$ and $\Psi_{2}$ form a basis for the solution set for $z \in \mathbb{R}$, so at $t=0$

$$
\Phi_{1}(z ; x, 0)=a(z ; 0) \Psi_{1}(z ; x, 0)+b(z ; 0) \Psi_{2}(z ; x, 0) .
$$

$a(z ; t)$ and $b(z ; t)$ are independent of $x$. Explicitly,

$$
a(z ; 0)=\frac{\left(\lambda_{R}+(z-\mu / 2)+\lambda_{L}-(z+\mu / 2)\right)}{2 \lambda_{R}}
$$

and

$$
b(z ; 0)=\frac{-\lambda_{R}-(z-\mu / 2)}{A} \frac{\lambda_{R}-(z-\mu / 2)-\lambda_{L}+(z+\mu / 2)}{2 \lambda_{R}} .
$$


Define the scattering coefficient $\rho(z)$ as

$$
\begin{aligned}
\rho(z) & =\frac{b(z ; 0)}{a(z ; 0)} \\
& =\frac{-\lambda_{R}-(z-\mu / 2)}{A} \frac{\left(\lambda_{R}-(z-\mu / 2)-\lambda_{L}+(z+\mu / 2)\right)}{\left(\lambda_{R}+(z-\mu / 2)+\lambda_{L}-(z+\mu / 2)\right)} .
\end{aligned}
$$

\section{A.3 Time Evolution of the Reflection Coefficient}

Let $\Phi_{1}^{(t)}(z ; x, t)$ be the solution to the equation $\partial_{t} \Phi_{1}^{(t)}=-i \widehat{C} \Phi_{1}^{(t)}$ with initial condition $\Phi_{1}(z ; x, 0)$.

$$
\widehat{C}=\left(z-\frac{\mu}{2}\right)\left[\begin{array}{cc}
z+\frac{\mu}{2} & A \\
A & -z-\frac{\mu}{2}
\end{array}\right] \quad \text { as } x \rightarrow-\infty,
$$

SO

$$
\Phi_{1}^{(t)}(z ; x, t)=e^{-i(z-\mu / 2) \lambda_{L} t}\left[\begin{array}{c}
1 \\
\frac{\lambda_{L}-(z+\mu / 2)}{A}
\end{array}\right] e^{-i \lambda_{L} x} \quad \text { as } x \rightarrow-\infty .
$$

$\Phi_{1}(z ; x, t)$ is normalized to be constant in time as $x \rightarrow-\infty$. Therefore

$$
\Phi_{1}(z ; x, t)=e^{i(z-\mu / 2) \lambda_{L} t} \Phi_{1}^{(t)}(z ; x, t) .
$$

Also, let $\Psi_{1}^{(t)}(z ; x, t)$ be the solution to the equation $\partial_{t} \Psi_{1}^{(t)}=-i \widehat{C} \Psi_{1}^{(t)}$ with initial condition $\Psi_{1}(z ; x, 0)$ and define $\Psi_{2}^{(t)}(z ; x, t)$ analogously.

$$
\widehat{C}=\left(z+\frac{\mu}{2}\right)\left[\begin{array}{cc}
z-\frac{\mu}{2} & A \\
A & -z+\frac{\mu}{2}
\end{array}\right] \quad \text { as } x \rightarrow \infty,
$$

so

$$
\Psi_{1}^{(t)}(z ; x, t)=e^{-i(z+\mu / 2) \lambda_{R} t}\left[\begin{array}{c}
1 \\
\frac{\lambda_{R}-(z-\mu / 2)}{A}
\end{array}\right] e^{-i \lambda_{R} x} \quad \text { as } x \rightarrow \infty
$$

and

$$
\Psi_{2}^{(t)}(z ; x, t)=e^{i(z+\mu / 2) \lambda_{R} t}\left[\begin{array}{c}
\frac{-\lambda_{R}+(z-\mu / 2)}{A} \\
1
\end{array}\right] e^{i \lambda_{R} x} \quad \text { as } x \rightarrow \infty .
$$

$\Psi_{1}(z ; x, t)$ and $\Psi_{2}(z ; x, t)$ are normalized to be constant in time as $x \rightarrow \infty$. Therefore

$$
\Psi_{1}(z ; x, t)=e^{i(z+\mu / 2) \lambda_{R} t} \Psi_{1}^{(t)}(z ; x, t)
$$

and

$$
\Psi_{2}(z ; x, t)=e^{-i(z+\mu / 2) \lambda_{R} t} \Psi_{2}^{(t)}(z ; x, t) .
$$

Now

$$
\Phi_{1}(z ; x, t)=a(z ; t) \Psi_{1}(z ; x, t)+b(z ; t) \Psi_{2}(z ; x, t)
$$

or

$$
e^{i(z-\mu / 2) \lambda_{L} t} \Phi_{1}^{(t)}=a e^{i(z+\mu / 2) \lambda_{R} t} \Psi_{1}^{(t)}+b e^{-i(z+\mu / 2) \lambda_{R} t} \Psi_{2}^{(t)} .
$$


As $x \rightarrow \infty$, since $\widehat{C}$ is constant,

$$
\begin{aligned}
& \Phi_{1}^{(t)}(x, t)=e^{-i \widehat{C} t} \Phi_{1}(x, 0), \\
& \Psi_{1}^{(t)}(x, t)=e^{-i \widehat{C} t} \Psi_{1}(x, 0), \\
& \Psi_{2}^{(t)}(x, t)=e^{-i \widehat{C} t} \Psi_{2}(x, 0) .
\end{aligned}
$$

Therefore, as $x \rightarrow \infty$,

(A.25) $e^{i(z-\mu / 2) \lambda_{L} t} \Phi_{1}(z ; x, 0)=$

$$
e^{i(z+\mu / 2) \lambda_{R} t} a \Psi_{1}(z ; x, 0)+e^{-i(z+\mu / 2) \lambda_{R} t} b \Psi_{2}(z ; x, 0) .
$$

Comparing this with

$$
\Phi_{1}(z ; x, 0)=a(z ; 0) \Psi_{1}(z ; x, 0)+b(z ; 0) \Psi_{2}(z ; x, 0)
$$

gives

$$
\begin{aligned}
& a(z ; t)=e^{i\left[-(z+\mu / 2) \lambda_{R}+(z-\mu / 2) \lambda_{L}\right] t} a(z ; 0), \\
& b(z ; t)=e^{i\left[(z+\mu / 2) \lambda_{R}+(z-\mu / 2) \lambda_{L}\right] t} b(z ; 0) .
\end{aligned}
$$

Note that the coefficient $a(z ; t)$ depends on $t$. Now

$$
r(z ; t)=\frac{b(z ; t)}{a(z ; t)}=e^{2 i(z+\mu / 2) \lambda_{R} t} \rho(z) .
$$

\section{A.4 The Fundamental Solution $M^{(0)}(z)$}

$a(z ; t), b(z ; t), \rho(z), \Phi_{1,2}(z ; x, t)$, and $\Psi_{1,2}(z ; x, t)$ can be extended analytically to the entire complex $z$-plane except for the $\lambda_{L}$ and $\lambda_{R}$ branch cuts. For $x>0$, choose the fundamental solution $M^{(0)}(z ; x, t)$ to be

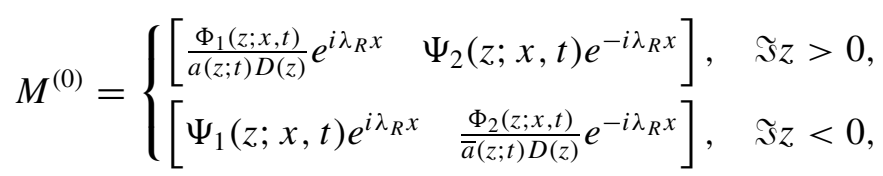

and for $x<0$ choose

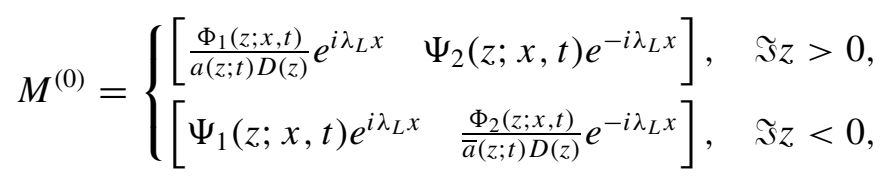

where

$$
D(z)=\operatorname{det}\left[\begin{array}{ll}
\Psi_{1} & \Psi_{2}
\end{array}\right]=\frac{2 \lambda_{R}^{2}-2(z-\mu / 2) \lambda_{R}}{A^{2}}
$$

is independent of $x$ and $t$ by equations (1.3) and (1.4) and Abel's theorem. The function $M^{(0)}(z ; x, t)$ is analytic except across the real $z$-axis and the branch cuts for $\lambda_{L}$ and $\lambda_{R}$, and is normalized to the identity matrix as $z \rightarrow \infty$. The factor of $D$ is included to ensure det $M^{(0)}=1$ for all $z$. For instance, for $x>0$ and $\Im z>0$,

(A.33) $\operatorname{det} M^{(0)}=\operatorname{det}\left[\frac{1}{D}\left(\Psi_{1}+r \Psi_{2}\right) e^{i \lambda_{R} x} \quad \Psi_{2} e^{-i \lambda_{R} x}\right]=\frac{1}{D} \operatorname{det}\left[\begin{array}{ll}\Psi_{1} & \Psi_{2}\end{array}\right]=1$. 
The determinant condition can be checked for $\Im z<0$ using the identity

$$
\Phi_{2}(z ; x, t)=-\bar{b}(z ; t) \Psi_{1}(z ; x, t)+\bar{a}(z ; t) \Psi_{2}(z ; x, t) .
$$

\section{A.5 Reconstructing $q(x, t)$ from $M^{(0)}(z)$}

$M^{(0)}$ in the upper half $z$-plane can be written as $U e^{i \lambda_{R} x \sigma_{3}}$, where

$$
U=\left[\frac{\Phi_{1}}{a(z ; t)}, \quad \Psi_{2}\right]
$$

satisfies equation (A.2). As $z \rightarrow \infty$, equation (A.2) becomes

$$
U_{x}=-i\left[\begin{array}{cc}
z & q e^{i\left[\mu|x|+\left(\mu^{2} / 2-A^{2}\right) t\right]} \\
\bar{q} e^{-i\left[\mu|x|+\left(\mu^{2} / 2-A^{2}\right) t\right]} & -z
\end{array}\right] U .
$$

Also,

$$
U=M^{(0)} e^{-i z x \sigma_{3}} .
$$

Therefore, as $z \rightarrow \infty, M^{(0)}$ satisfies

$$
M_{x}^{(0)}-i z M^{(0)} \sigma_{3}=-i\left[\begin{array}{cc}
z & q e^{i\left[\mu|x|+\left(\mu^{2} / 2-A^{2}\right) t\right]} \\
\bar{q} e^{-i\left[\mu|x|+\left(\mu^{2} / 2-A^{2}\right) t\right]} & -z
\end{array}\right] M^{(0)} .
$$

Write $M^{(0)}$ at infinity in a Laurent series as

$$
M^{(0)}=I+z^{-1} M_{1}^{(0)}+z^{-2} M_{2}^{(0)}+\cdots,
$$

where the $M_{i}^{(0)}$ are independent of $z$. Looking at the 12-entry of this matrix equation and matching terms of order unity gives

$$
q(x, t)=-2\left(M_{1}^{(0)}\right)_{12} e^{-i\left[\mu|x|+\left(\mu^{2} / 2-A^{2}\right) t\right]},
$$

where $\left(M_{1}^{(0)}\right)_{12}$ is the 12-entry of $M_{1}^{(0)}$.

\section{Appendix B: The Plane-Wave Region Parametrix}

This appendix outlines the construction of $M^{(\mathrm{app})}$ inside $r_{z_{0}}^{\varepsilon}$ for the plane-wave region. The idea is to use a locally analytic transformation $T: z \rightarrow \hat{z}$ to simplify the phase function $f$. The parametrix is then constructed via a method used by Deift and Zhou [9] for the defocusing NLS.

\section{B.1 Reduction to a Constant RHP $\boldsymbol{P}^{(\psi)}$}

By the definition of $z_{0}$ and the Cauchy-Riemann equations, $z_{0}$ is a stationary point of $f(z)$, so $f^{\prime}\left(z_{0}\right)=0$. Therefore $f(z)$ can be written in a Taylor series around $z_{0}$ as

$$
f(z)=f_{0}+\sum_{n=2}^{\infty} f_{n}\left(z-z_{0}\right)^{n}
$$




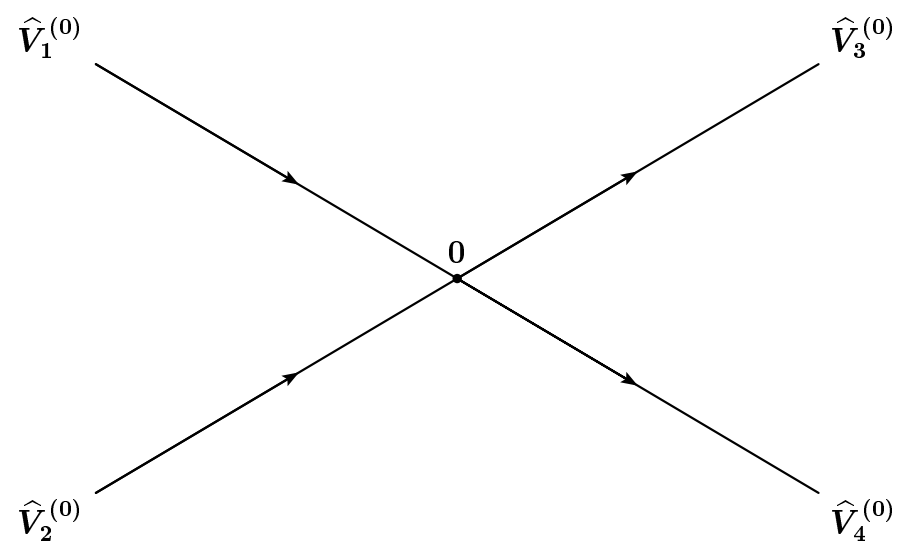

FIgURE B.1. The RHP $\widehat{P}^{(0)}$ for the plane-wave region.

where the $f_{i}$ depend only on $A, \mu$, and $\xi$. In $D_{z_{0}}^{\varepsilon}$, define $T: z \rightarrow \hat{z}$ by

$$
\hat{z}=\sqrt{\frac{t}{2}}\left(z-z_{0}\right)\left(\sum_{n=0}^{\infty} f_{n+2}\left(z-z_{0}\right)^{n}\right)^{1 / 2} .
$$

Then $T$ is biholomorphic in $D_{z_{0}}^{\varepsilon}$, and $T\left(e^{i f(z) t}\right)=e^{i f_{0} t} e^{2 i \hat{z}^{2}}$.

The transformed RHP is called $\widehat{P}^{(0)}$. In $T\left(D_{z_{0}}^{\varepsilon}\right), \widehat{M}^{(0)}$ needs to satisfy the jump conditions of $T\left(V^{(3)}\right)$. The solution outside $T\left(r_{z 0}^{\varepsilon}\right)$ is not used, so we are free to choose the contour, jumps, and normalization. For the contour choose two straight lines through $\hat{z}=0$ that do not intersect $T\left(\lambda_{L}\right.$ cut) or $T\left(\lambda_{R}\right.$ cut) (see Figure B.1). This contour is independent of $t$. The RHP is written as

$$
\widehat{P}^{(0)}:\left\{\widehat{\Sigma}^{(0)}, \widehat{V}^{(0)}, I \text { as } \hat{z} \rightarrow \infty\right\},
$$

with

$$
\widehat{V}_{i}^{(0)}=T\left(V_{i}^{(3)}\right), \quad i=1,2,3,4 .
$$

To write down formulae for $\widehat{V}^{(0)}$, we express $z$ in terms of $\hat{z}$. Starting with equation (B.2), we see that near $z_{0}$ we can express $\hat{z} / \sqrt{t}$ as a series as

$$
\frac{\hat{z}}{\sqrt{t}}=\sum_{n=1}^{\infty} \alpha_{n}\left(z-z_{0}\right)^{n},
$$

where the known coefficients $\alpha_{i}$ depend only on $A, \mu$, and $\xi$. We also write $z-z_{0}$ as a series in $\hat{z} / \sqrt{t}$ :

$$
z-z_{0}=\sum_{n=1}^{\infty} \beta_{n}\left(\frac{\hat{z}}{\sqrt{t}}\right)^{n}
$$


Now substitute equation (B.5) into equation (B.4) and match powers of $\hat{z} / \sqrt{t}$. This yields an infinite system of polynomial equations of the form

$$
\alpha_{1} \beta_{k}+P_{k}\left(\beta_{k-1}, \beta_{k-2}, \ldots, \beta_{1}\right)=0,
$$

where $P_{k}$ is a polynomial with coefficients depending on $\alpha_{1}, \ldots, \alpha_{k}$. Therefore the $\beta_{k}$ can be solved for recursively in terms of known quantities starting with $\beta_{1}=1 / \alpha_{1}$.

Given a function $F(z)$, define $\widehat{F}(\hat{z})$ by

$$
\widehat{F}(\hat{z})=F\left(z_{0}+\sum_{n=1}^{\infty} \beta_{n}\left(\frac{\hat{z}}{\sqrt{t}}\right)^{n}\right) .
$$

Using integration by parts, $\hat{\delta}$ can be written

$$
\hat{\delta}(\hat{z})=\exp \left\{v\left[\ln \left(\frac{\hat{z}}{\sqrt{t}}\right)+\ln \beta_{1}+\ln \left(1+\sum_{n=2}^{\infty} \frac{\beta_{n}}{\beta_{1}}\left(\frac{\hat{z}}{\sqrt{t}}\right)^{n-1}\right)\right]+\chi(\hat{z})\right\}
$$

wherein

$$
\begin{aligned}
v & =\frac{1}{2 \pi i} \ln \left(1+\rho^{2}\left(z_{0}\right)\right), \\
\chi(\hat{z}) & =-\frac{1}{2 \pi i} \int_{-\infty}^{z_{0}} \ln \left(-\zeta+z_{0}+\sum_{n=1}^{\infty} \beta_{n}\left(\frac{\hat{z}}{\sqrt{t}}\right)^{n}\right) \mathrm{d} \ln \left(1+\rho^{2}(\zeta)\right) .
\end{aligned}
$$

Now write

$$
\hat{\delta} e^{-i(\widehat{f} t / 2+\widehat{g})}=\hat{\delta}^{0} \hat{\delta}^{1},
$$

where $\hat{\delta}^{0}$ and $\hat{\delta}^{1}$ are defined by

$$
\begin{array}{r}
\hat{\delta}^{0}=t^{-v / 2} \exp \left[v \ln \beta_{1}+\chi(0)-i f_{0} \frac{t}{2}-i \widehat{g}(0)\right], \\
\hat{\delta}^{1}(\hat{z})=\hat{z}^{v} \exp \left[-i \hat{z}^{2}+\ln \left(1+\sum_{n=2}^{\infty} \frac{\beta_{n}}{\beta_{1}}\left(\frac{\hat{z}}{\sqrt{t}}\right)^{n-1}\right)\right. \\
+\chi(\hat{z})-\chi(0)-i \widehat{g}(\hat{z})+i \widehat{g}(0)] .
\end{array}
$$

So $\widehat{V}^{(0)}$ can be written as

$$
\begin{array}{ll}
\widehat{V}_{1}^{(0)}=\left[\begin{array}{cc}
1 & \left(\hat{\delta}^{0} \hat{\delta}^{1}\right)^{2} \frac{\hat{\rho}}{1+\hat{\rho}^{2}} \\
0 & 1
\end{array}\right], & \widehat{V}_{2}^{(0)}=\left[\begin{array}{ccc}
1 & 0 \\
\left(\hat{\delta}^{0} \hat{\delta}^{1}\right)^{-2} \frac{\hat{\rho}}{1+\hat{\rho}^{2}} & 1
\end{array}\right], \\
\widehat{V}_{3}^{(0)}=\left[\begin{array}{ccc}
1 & 0 \\
\left(\hat{\delta}^{0} \hat{\delta}^{1}\right)^{-2} \hat{\rho} & 1
\end{array}\right], & \widehat{V}_{4}^{(0)}=\left[\begin{array}{cc}
1 & \left(\hat{\delta}^{0} \hat{\delta}^{1}\right)^{2} \hat{\rho} \\
0 & 1
\end{array}\right] .
\end{array}
$$

To factor out the $\hat{z}$-independent $\hat{\delta}^{0}$, introduce

$$
\widehat{M}^{(1)}=\left(\hat{\delta}^{0}\right)^{-\sigma_{3}} \widehat{M}^{(0)}\left(\hat{\delta}^{0}\right)^{\sigma_{3}} .
$$




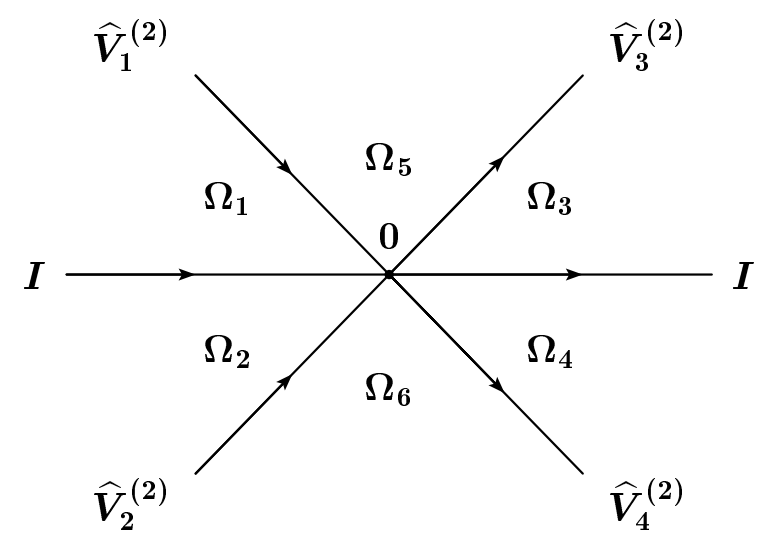

FIGURE B.2. The RHP $\widehat{P}^{(2)}$ for the plane-wave region.

$\widehat{M}^{(1)}$ satisfies the RHP

$$
\widehat{P}^{(1)}:\left\{\widehat{\Sigma}^{(1)}=\widehat{\Sigma}^{(0)}, \widehat{V}^{(1)}=\left(\hat{\delta}^{0}\right)^{-\sigma_{3}} \widehat{V}^{(0)}\left(\hat{\delta}^{0}\right)^{\sigma_{3}}, I \text { as } \hat{z} \rightarrow \infty\right\} .
$$

The jump matrix $\widehat{V}^{(1)}$ is given explicitly by

$$
\begin{array}{ll}
\widehat{V}_{1}^{(1)}=\left[\begin{array}{cc}
1 & \left(\hat{\delta}^{1}\right)^{2} \frac{\hat{\rho}}{1+\hat{\rho}^{2}} \\
0 & 1
\end{array}\right], & \widehat{V}_{2}^{(1)}=\left[\begin{array}{ccc}
1 & 0 \\
\left(\hat{\delta}^{1}\right)^{-2} \frac{\hat{\rho}}{1+\hat{\rho}^{2}} & 1
\end{array}\right], \\
\widehat{V}_{3}^{(1)}=\left[\begin{array}{ccc}
1 & 0 \\
\left(\hat{\delta}^{1}\right)^{-2} \hat{\rho} & 1
\end{array}\right], & \widehat{V}_{4}^{(1)}=\left[\begin{array}{cc}
1 & \left(\hat{\delta}^{1}\right)^{2} \hat{\rho} \\
0 & 1
\end{array}\right] .
\end{array}
$$

As $t \rightarrow \infty, \hat{\rho}(\hat{z})$ and $\hat{\delta}^{1}(\hat{z})$ tend uniformly in $t$ to $\hat{\rho}(0)$ and $\hat{z}^{v} e^{-i \hat{z}^{2}}$, respectively. Thus for large times, $\widehat{V}^{(1)} \sim \widehat{V}^{(2)}$ where $\widehat{V}^{(2)}$ is independent of $t$. Augment the contour $\widehat{\Sigma}^{(1)}$ by adding the real axis oriented left to right with jump equal to the identity. This divides the $\hat{z}$-plane into six regions $\Omega_{1}, \ldots, \Omega_{6}$, as shown in Figure B.2. $\widehat{M}^{(2)}$ is defined to be the solution to the RHP

$$
\widehat{P}^{(2)}:\left\{\widehat{\Sigma}^{(2)}=\widehat{\Sigma}^{(1)} \cup \mathbb{R}, \widehat{V}^{(2)}, I \text { as } \hat{z} \rightarrow \infty\right\},
$$

where

$$
\begin{array}{ll}
\widehat{V}_{1}^{(2)}=\left[\begin{array}{cc}
1 & \hat{z}^{2 v} e^{-2 i \hat{z}^{2}} \frac{\hat{\rho}(0)}{1+\hat{\rho}^{2}(0)} \\
0 & 1
\end{array}\right], \quad \widehat{V}_{2}^{(2)}=\left[\begin{array}{cc}
1 & 0 \\
\hat{z}^{-2 v} e^{2 i \hat{z}^{2}} \frac{\hat{\rho}(0)}{1+\hat{\rho}^{2}(0)} & 1
\end{array}\right], \\
\widehat{V}_{3}^{(2)}=\left[\begin{array}{ccc}
1 & 0 \\
\hat{z}^{-2 v} e^{2 i \hat{z}^{2}} \hat{\rho}(0) & 1
\end{array}\right], & \widehat{V}_{4}^{(2)}=\left[\begin{array}{cc}
1 & \hat{z}^{2 v} e^{-2 i \hat{z}^{2}} \hat{\rho}(0) \\
0 & 1
\end{array}\right], \\
\widehat{V}_{5}^{(2)}=\widehat{V}_{6}^{(2)}=I . &
\end{array}
$$

From Deift and Zhou [9], 


$$
\widehat{M}_{1}^{(1)}=\widehat{M}_{1}^{(2)}+O\left(t^{-1 / 2} \ln t\right) .
$$

Note that $\hat{\rho}(\hat{z})$ and $\widehat{f}(\hat{z})$ no longer appear in the jump matrices $\widehat{V}^{(2)}$. Therefore the barriers to deforming the contours back to the real axis have been removed. Define the function

$$
\phi\left(\hat{z} .= \begin{cases}\hat{z}^{-v \sigma_{3}}\left(\widehat{V}_{i}^{(2)}\right)^{-1}, & \Omega_{i}, i=1,3, \\ \hat{z}^{-v \sigma_{3}} \widehat{V}_{i}^{(2)} & \Omega_{i}, i=2,4, \\ \hat{z}^{-v \sigma_{3}}, & \Omega_{5} \cup \Omega_{6} .\end{cases}\right.
$$

Also define $\widehat{M}^{(3 .}=\widehat{M}^{(2)} \phi^{-1}$ as the solution to the RHP

$$
\widehat{P}^{(3 .}:\left\{\widehat{\Sigma}^{(3 .}, \widehat{V}^{(3 .}, \hat{z}^{v \sigma_{3}} \text { as } \hat{z} \rightarrow \infty\right\} .
$$

Observe that $\hat{z}^{v}$ has a jump discontinuity on the negative real axis, and therefore

$$
\left(\frac{\hat{z}_{+}}{\hat{z}_{-}}\right)^{v}=\frac{e^{\nu(\ln |\hat{z}|+i \pi)}}{e^{\nu(\ln |\hat{z}|-i \pi)}}=e^{2 i \pi v}=1+\hat{\rho}^{2}(0) \quad \text { on }(-\infty, 0) .
$$

Using this yields

$$
\widehat{V}_{i}^{(3)}= \begin{cases}e^{-i \hat{z}^{2} \mathrm{ad} \sigma_{3}}\left[\begin{array}{cc}
1+\hat{\rho}^{2}(0) & \hat{\rho}(0) \\
\hat{\rho}(0) & 1
\end{array}\right], \quad i=5,6, \\
I, & i=1,2,3,4 .\end{cases}
$$

We arrive at a constant-jump RHP on the real axis by defining

$$
\psi=\widehat{M}^{(3)} e^{-i \hat{z}^{2} \sigma_{3}} \text {. }
$$

$\psi$ satisfies the Riemann-Hilbert problem

$$
P^{(\psi)}:\left\{\Sigma^{(\psi)}=\mathbb{R}, V^{(\psi)}, e^{-i \hat{z}^{2} \sigma_{3}} \hat{z}^{\nu \sigma_{3}} \text { as } \hat{z} \rightarrow \infty\right\} .
$$

\section{B.2 Solution of the RHP $P^{(\psi)}$}

Starting with $\psi_{+}=\psi_{-} V^{(\psi)}$, it follows that

$$
\left(\partial_{\hat{z}} \psi+2 i \hat{z} \sigma_{3} \psi\right)_{+}=\left(\partial_{\hat{z}} \psi+2 i \hat{z} \sigma_{3} \psi\right)_{-} V^{(\psi)} .
$$

Since $\left(\partial_{\hat{z}} \psi+2 i \hat{z} \sigma_{3} \psi\right)$ and $\psi$ have the same jump across $\mathbb{R},\left(\partial_{\hat{z}} \psi+2 i \hat{z} \sigma_{3} \psi\right) \psi^{-1}$ is analytic everywhere in the complex plane. Define

$$
\tilde{\psi}=\hat{z}^{-v \sigma_{3}} e^{i z^{2} \sigma_{3}} \psi \text {. }
$$

Now because $\operatorname{det}(\tilde{\psi})=\operatorname{det}(\psi)=1$ for all $\hat{z}, \tilde{\psi}^{-1}$ exists and

$$
\tilde{\psi}^{-1}=I-\hat{z}^{-1} \widehat{M}_{1}^{(2)}+O\left(\hat{z}^{-2}\right) \quad \text { as } \hat{z} \rightarrow \infty .
$$

This gives

(B.25) $\left(\partial_{\hat{z}} \psi+2 i \hat{z} \sigma_{3} \psi\right) \psi^{-1}$

$$
\begin{aligned}
& =\left(\partial_{\hat{z}}\left(\tilde{\psi} \hat{z}^{v \sigma_{3}} e^{-i \hat{z}^{2} \sigma_{3}}\right)+2 i \hat{z} \sigma_{3} \tilde{\psi} \hat{z}^{v \sigma_{3}} e^{-i \hat{z}^{2} \sigma_{3}}\right) e^{i \hat{z}^{2} \sigma_{3} \hat{z}^{-v \sigma_{3}}(\tilde{\psi})^{-1}} \\
& =\left(\partial_{\hat{z}} \tilde{\psi}\right)(\tilde{\psi})^{-1}+v \hat{z}^{-1} \tilde{\psi} \sigma_{3}(\tilde{\psi})^{-1}-2 i \hat{z} \tilde{\psi} \sigma_{3}(\tilde{\psi})^{-1}+2 i \hat{z} \sigma_{3} \tilde{\psi}(\tilde{\psi})^{-1}
\end{aligned}
$$




$$
\begin{aligned}
& =2 i \hat{z}\left[\sigma_{3}, \tilde{\psi}\right](\tilde{\psi})^{-1}+O\left(\hat{z}^{-1}\right) \\
& =2 i \hat{z}\left[\sigma_{3}, I+\hat{z}^{-1} \widehat{M}_{1}^{(2)}+O\left(\hat{z}^{-2}\right)\right]\left(I-\hat{z}^{-1} \widehat{M}_{1}^{(2)}+O\left(\hat{z}^{-2}\right)\right)+O\left(\hat{z}^{-1}\right) \\
& =2 i\left[\sigma_{3}, \widehat{M}_{1}^{(2)}\right]+O\left(\hat{z}^{-1}\right) .
\end{aligned}
$$

This is bounded in $\hat{z}$, so $\left(\partial_{\hat{z}} \psi+2 i \hat{z} \sigma_{3} \psi\right) \psi^{-1}$ is bounded and entire. By Liouville's theorem,

$$
\partial_{\hat{z}} \psi+2 i \hat{z} \sigma_{3} \psi=2 i\left[\sigma_{3}, \widehat{M}_{1}^{(2)}\right] \psi=4 i\left[\begin{array}{cc}
0 & \left(\widehat{M}_{1}^{(2)}\right)_{12} \\
-\left(\widehat{M}_{1}^{(2)}\right)_{21} & 0
\end{array}\right] \psi .
$$

Define

$$
\beta_{12}=4 i\left(\widehat{M}_{1}^{(2)}\right)_{12}, \quad \beta_{21}=-4 i\left(\widehat{M}_{1}^{(2)}\right)_{21} .
$$

Now, two of the components of equation (B.26) yield the system

$$
\begin{aligned}
& \partial_{\hat{z}} \psi_{11}+2 i \hat{z} \psi_{11}=\beta_{12} \psi_{21}, \\
& \partial_{\hat{z}} \psi_{21}-2 i \hat{z} \psi_{21}=\beta_{21} \psi_{11},
\end{aligned}
$$

which reduces to

$$
\partial_{\hat{z}}^{2} \psi_{11}+\left(4 \hat{z}^{2}+2 i-\beta_{12} \beta_{21}\right) \psi_{11}=0 .
$$

Let $\psi^{+}$be the solution $\psi$ for $\mathfrak{\Im} \hat{z}>0$ and $\psi^{-}$the solution for $\mathfrak{\Im} \hat{z}<0$. Set

$$
\psi_{11}^{+}(\hat{z})=j\left(e^{-3 i \pi / 4} \hat{z}\right)=j(\zeta) .
$$

$j(\zeta)$ satisfies the standard parabolic cylinder equation

$$
\partial_{\zeta}^{2} j+\left(\frac{1}{2}-\frac{\zeta^{2}}{4}+a\right) j=0
$$

for $a=\frac{i}{4} \beta_{12} \beta_{21}$. This means

$$
\psi_{11}^{+}(\hat{z})=c_{1} D_{a}\left(2 e^{-3 i \pi / 4} \hat{z}\right)+c_{2} D_{a}\left(-2 e^{-3 i \pi / 4} \hat{z}\right)
$$

for some constants $c_{1}$ and $c_{2}$. The asymptotics of the parabolic cylinder function $D_{a}(\zeta)$ are given in Abramowitz and Stegun [2] as

$$
D_{a}(\zeta)=\zeta^{a} e^{-\zeta^{2} / 4}\left(1+O\left(\zeta^{-2}\right)\right), \quad \zeta \rightarrow \infty,|\arg \zeta|<\frac{3 \pi}{4} .
$$

Also, from the asymptotics for $\psi$, for $\zeta>0$,

$$
\psi_{11}^{+}\left(\frac{1}{2} e^{3 i \pi / 4} \zeta\right)=\left(\frac{1}{2} e^{3 i \pi / 4}\right)^{v} \zeta^{\nu} e^{-\zeta^{2} / 4}\left(1+O\left(\zeta^{-1}\right)\right) \quad \text { as } \zeta \rightarrow \infty .
$$

Therefore by matching along the positive real axis, $a=v, c_{1}=\left(\frac{1}{2} e^{3 i \pi / 4}\right)^{v}$, and $c_{2}=0$. So

$$
\psi_{11}^{+}=\left(\frac{1}{2} e^{3 i \pi / 4}\right)^{\nu} D_{a}\left(2 e^{-3 i \pi / 4} \hat{z}\right)
$$


From equation (B.28),

$$
\psi_{21}^{+}=\beta_{12}^{-1}\left(\partial_{\hat{z}} \psi_{11}^{+}+2 i \hat{z} \partial_{11}^{+}\right)
$$

$$
=\beta_{12}^{-1}\left(\frac{1}{2} e^{3 i \pi / 4}\right)^{v}\left[\partial_{\hat{z}} D_{v}\left(2 e^{-3 i \pi / 4} \hat{z}\right)+2 i \hat{z} D_{v}\left(2 e^{-3 i \pi / 4} \hat{z}\right)\right] .
$$

Similar computations are done in the lower half-plane. Start by defining

$$
k\left(2 e^{i \pi / 4}\right)=\psi_{11}^{-}(\hat{z}) .
$$

The end result is

$$
\psi_{11}^{-}=\left(\frac{1}{2} e^{-i \pi / 4}\right)^{v} D_{a}\left(2 e^{i \pi / 4} \hat{z}\right)
$$

and

$$
\psi_{21}^{-}=\beta_{12}^{-1}\left(\frac{1}{2} e^{-i \pi / 4}\right)^{v}\left[\partial_{\hat{z}} D_{v}\left(2 e^{i \pi / 4} \hat{z}\right)+2 i \hat{z} D_{v}\left(2 e^{i \pi / 4} \hat{z}\right)\right] .
$$

From the jump condition, $\left(\psi^{-}\right)^{-1} \psi^{+}=V^{(\psi)}$, so

$$
\begin{aligned}
\hat{\rho}(0) & =\psi_{11}^{-} \psi_{21}^{+}-\psi_{11}^{+} \psi_{21}^{-} \\
& =\left(\frac{1}{2} e^{i \pi / 4}\right)^{2 v} \beta_{12}^{-1} \text { Wronskian }\left[D_{v}\left(2 e^{i \pi / 4} \hat{z}\right), D_{v}\left(2 e^{-3 i \pi / 4} \hat{z}\right)\right] \\
& =\frac{(2 \pi)^{1 / 2} 2 e^{i \pi / 4}\left(\frac{1}{2} e^{i \pi / 4}\right)^{2 v}}{\beta_{12} \Gamma(-v)},
\end{aligned}
$$

from which it is possible to express $\beta_{12}$ in terms of known quantities. Explicit formulae for $\psi_{12}$ and $\psi_{22}$ may be obtained in an analogous manner.

\section{B.3 Computation of $V_{z_{0}}^{(\text {err })}$}

Now that $\psi$ is known, $\widehat{M}^{(0)}$ is given by

$$
\widehat{M}^{(0)}=\left(\hat{\delta}^{0}\right)^{\sigma_{3}} \psi e^{i \hat{z}^{2} \sigma_{3}} \phi\left(\hat{\delta}^{0}\right)^{-\sigma_{3}}+O\left(t^{-1 / 2} \ln t\right) .
$$

We are interested in the behavior of $\widehat{M}^{(0)}$ on $T\left(r_{z_{0}}^{\varepsilon}\right)$. As $t$ approaches $\infty, \hat{z} \in T\left(r_{z_{0}}^{\varepsilon}\right)$ also tends to infinity. By equations (B.14) and (B.17), for large $t$

$$
\begin{aligned}
\widehat{M}^{(0)} & =I+\frac{1}{\hat{z}} \widehat{M}_{1}^{(0)}+O\left(\hat{z}^{-2}\right) \\
& =I+\frac{1}{\hat{z}}\left(\hat{\delta}^{0}\right)^{\sigma_{3}}\left(\widehat{M}_{1}^{(2)}+O\left(t^{-1 / 2} \ln t\right)\right)\left(\hat{\delta}^{0}\right)^{-\sigma_{3}}+O\left(\hat{z}^{-2}\right)
\end{aligned}
$$

on $T\left(r_{z 0}^{\varepsilon}\right)$. Switching back to $z$ using (B.4), we have

$$
T^{-1}\left(\widehat{M}^{(0)}\right)=I+O\left(t^{-1 / 2}\right)
$$

on $r_{z_{0}}^{\varepsilon} \cdot M^{(\mathrm{mod})}$ is analytic near $z_{0}$, so define

$$
M^{(\mathrm{app})}=M^{(\mathrm{mod})} T^{-1}\left(\widehat{M}^{(0)}\right) \text { inside } r_{z 0}^{\varepsilon} .
$$


By the definition of $M^{(\text {app })}$ in (2.33), $V_{z_{0}}^{(\text {app })}=I+O\left(t^{-1 / 2}\right)$ for large $t$. By equation (2.35),

$$
V_{z_{0}}^{(\mathrm{err})}=I+O\left(t^{-1 / 2}\right)
$$

for large $t$.

Acknowledgment. The authors would like to thank Xin Zhou and Peter Miller for many useful conversations. Stephanos Venakides thanks the National Science Foundation for supporting this research under Grant DMS-0207262.

\section{Bibliography}

[1] Ablowitz, M. J.; Kaup, D. J.; Newell, A. C.; Segur, H. The inverse scattering transform-Fourier analysis for nonlinear problems. Studies in Appl. Math. 53 (1974), no. 4, 249-315.

[2] Abramowitz, M.; Stegun, I. Handbook of mathematical functions with formulas, graphs, and mathematical tables. Dover, New York, 1966.

[3] Beals, R.; Coifman, R. Scattering and inverse scattering for first order systems. Comm. Pure Appl. Math. 37 (1984), no. 1, 39-90.

[4] Deift, P. Orthogonal polynomials and random matrices: a Riemann-Hilbert approach. Courant Lecture Notes in Mathematics, 3. New York University, Courant Institute of Mathematical Sciences, New York; American Mathematical Society, Providence, R.I., 1999.

[5] Deift, P.; Kriecherbauer, T.; McLaughlin, K. T.-R.; Venakides, S.; Zhou, X. Uniform asymptotics for polynomials orthogonal with respect to varying exponential weights and applications to universality questions in random matrix theory. Comm. Pure Appl. Math. 52 (1999), no. 11, 1335-1425.

[6] Deift, P.; Venakides, S.; Zhou, X. The collisionless shock region for the long-time behavior of solutions of the KdV equation. Comm. Pure Appl. Math. 47 (1994), no. 2, 199-206.

[7] Deift, P.; Venakides, S.; Zhou, X. New results in small dispersion KdV by an extension of the steepest descent method for Riemann-Hilbert problems. Internat. Math. Res. Notices 1997, no. 6, 285-299.

[8] Deift, P.; Zhou, X. A steepest descent method for oscillatory Riemann-Hilbert problems. Asymptotics for the MKdV equation. Ann. of Math. (2) 137 (1993), no. 2, 295-368.

[9] Deift, P.; Zhou, X. Long-time behavior of the non-focusing nonlinear Schrödinger equationa case study. New Series, Lectures in Mathematical Sciences, 5. University of Tokyo, Tokyo, 1995.

[10] Deift, P.; Zhou, X. Asymptotics for the Painlevé II equation. Comm. Pure Appl. Math. 48 (1995), 277-337.

[11] Farkas, H.; Kra, I. Riemann surfaces. 2nd ed. Graduate Texts in Mathematics, 71. Springer, New York, 1992.

[12] Gardner, C.; Greene, J.; Kruskal, M.; Miura, R. Method for solving the Korteweg-de Vries equation. Phys. Rev. Lett. 19 (1967), 1095-1097.

[13] Lax, P. Integrals of nonlinear equations of evolution and solitary waves. Comm. Pure Appl. Math. 21 (1968), 467-490.

[14] Shabat, A. One-dimensional perturbations of a differential operator, and the inverse scattering problem. Problems in mechanics and mathematical physics, 279-296. Nauka, Moscow, 1976.

[15] Tovbis, A.; Venakides, S.; Zhou, X. On semiclassical (zero dispersion limit) solutions of the focusing nonlinear Schrödinger equation. Comm. Pure Appl. Math. 57 (2004), 877-985.

[16] Venakides, S.; Deift, P.; Oba, R. The Toda shock problem. Comm. Pure Appl. Math. 44 (1991), $1171-1242$. 
[17] Zakharov, V.; Shabat, A. Exact theory of two-dimensional self-focusing and one-dimensional self-modulation of waves in nonlinear media. Soviet Physics JETP 34 (1972), no. 1, 62-69; translated from Ž. Èksper. Teoret. Fiz. 61 (1971), no. 1, 118-134.

\section{ROBERT BUCKINGHAM}

University of Michigan

Department of Mathematics

2074 East Hall

530 Church Street

Ann Arbor, MI 48109-1043

E-mail: robbiejb@umich.edu

Received October 2005.

\section{Stephanos Venakides}

Duke University

Department of Mathematics

Box 90320

Durham, NC 27708-0320

E-mail: ven@math.duke.edu 